\title{
EXPERIMENTS ON DYNAMIC AEROELASTIC RESPONSE OF WIND TURBINE BLADES
}

\author{
A Dissertation \\ by \\ ROBERT RAYMOND LONG \\ Submitted to the Office of Graduate and Professional Studies of \\ Texas A\&M University \\ in partial fulfillment of the requirements for the degree of \\ DOCTOR OF PHILOSOPHY
}

$\begin{array}{ll}\text { Chair of Committee, } & \text { Edward White } \\ \text { Committee Members, } & \text { Thomas Strganac } \\ & \text { John Valasek } \\ & \text { Reza Langari } \\ \text { Head of Department, } & \text { Rodney Bowersox }\end{array}$

December 2017

Major Subject: Aerospace Engineering

Copyright 2017 Robert R. Long 


\begin{abstract}
Wind turbine blades are growing progressively larger and lighter relative to their size, and these trends can lead to early failure due to fatigue. Effort to design fatigue-resistant blades relies on understanding of aeroelasticity - the study of unsteady interaction of structural, inertial, aerodynamic phenomenon.

The goal of this work is to provide needed wind tunnel experiments targeted at aeroelastic phenomenon relevant to wind turbine blades. The design of a wind tunnel test platform consisting of a rigid, cantilevered blade mounted to an elastic base, capable of flap and twist motion, is presented. The parameters of this model blade are intended to match the dynamic aeroelastic response of a reference design for a large-scale, megawatt-class wind turbine blade. A time-domain predictive model, based on 3D unsteady aerodynamics, is presented to simulate the aeroelastic response of the test platform subjected to arbitrary aerodynamic disturbances. Experimental validation of the predictive model is presented, and comparisons between different aerodynamics models are performed to identify regions of validity. From the experiments and model, conditions of interest to the study of fatigue are identified.

Experimental responses to periodic disturbances with a reduced frequency ranging from 0.098 to 0.56 are measured and compared to simulations. The simulated spanwise lift distribution agrees well with the measurements over the entire frequency range. The simulated flapwise deflection angles agree well with measurements when reduced frequency is less than 0.25 . This simulation model, and the accompanying test article, will serve as a platform for future research into wind turbine aeroelasticity and active control.
\end{abstract}




\section{DEDICATION}

To my family,

you raise me up on floppy wings. 


\section{ACKNOWLEDGMENTS}

I extend my sincere gratitude to all my committee members for their guidance and support. Particularly, I would like to thank Dr. Edward White, who has served as my mentor and role model since my undergraduate years. Our conversations, both on and off topic, gave me the motivation and confidence to conduct this research.

This work would not have been possible without the support of the staff at the Oran Nicks Low Speed Wind Tunnel. Thanks to John Kochan and Rick Warren for model installation and tunnel operation. Without John's help, I would not have been able to integrate my shoddy electronics into the data acquisition system. Thanks to Lisa Brown, who helped me with many tasks, including the setup of the tubing calibration system.

Finally, I'd like to extend a special thanks to my labmates, whose conversations helped me when I got stuck; my friends, who challenge and inspire me; my sisters, who keep me goofy; my parents, whose endless pride in me is almost embarrassing; and my wife, for all her love and patience. 


\section{CONTRIBUTORS AND FUNDING SOURCES}

\section{Contributors}

This work was supported by a dissertation committee consisting of Professors Edward White, Thomas Strganac, and John Valasek of the Department of Aerospace Engineering and Professor Reza Langari of the Department of Mechanical Engineering.

All work conducted for the dissertation was completed by the student independently.

\section{Funding Sources}

There are no outside funding contributions to acknowledge related to the research and copilation of this document. 


\section{TABLE OF CONTENTS}

Page

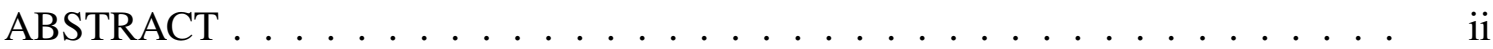

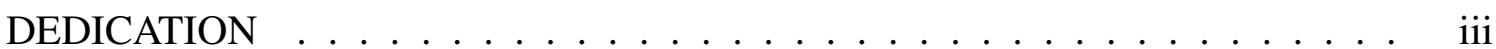

ACKNOWLEDGMENTS ...................... . . iv

CONTRIBUTORS AND FUNDING SOURCES . . . . . . . . . . . . v v

TABLE OF CONTENTS . . . . . . . . . . . . . . . . . . . . vi

LIST OF FIGURES . . . . . . . . . . . . . . . . . . . . . viii

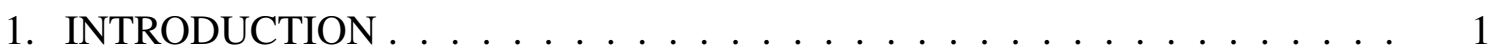

1.1 Wind Turbine Aerodynamics . . . . . . . . . . . . . . . . . 2

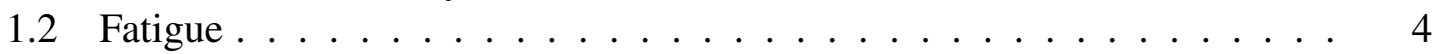

1.3 Objectives .......................... 7

2. EXPERIMENT DESIGN . . . . . . . . . . . . . . . . . . . . . . 9

2.1 Model Reduction . . . . . . . . . . . . . . . . . . . . . 10

2.2 Dynamic Aeroelastic Scaling . . . . . . . . . . . . . . . . 12

2.3 Rigid Blade . . . . . . . . . . . . . . . . . 16

2.4 Flexible Base . . . . . . . . . . . . . . . . . . . . 17

2.5 Pitch Actuator . . . . . . . . . . . . . . . . . 18

2.6 Tubing Correction . . . . . . . . . . . . . . . . . 20

2.7 Aerodynamic Measurements . . . . . . . . . . . . . . 22

2.8 Data Acquisition . . . . . . . . . . . . . . . . . 26

3. PREDICTIVE MODELS . . . . . . . . . . . . . . . . . . 29

3.1 Unsteady 2D Thin Airfoils . . . . . . . . . . . . . . . . . . . 29

3.2 Unsteady Lifting Line Theory . . . . . . . . . . . . . . . . . . . . . . . . . . . . . . .

3.3 Simulation of Motion . . . . . . . . . . . . . . . . 36

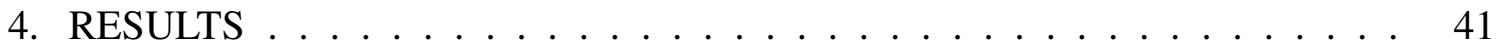

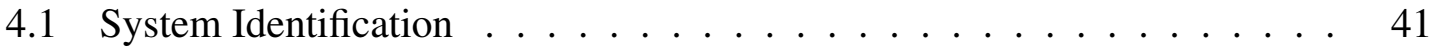


4.2 Static Pitch Sweep . . . . . . . . . . . . . . . . . . . . . . . . 45

4.3 Pitch Oscillations Tests . . . . . . . . . . . . . . . . . . . 55

4.3.1 Baseline Case . . . . . . . . . . . . . . . . . . 56

4.3.2 Effect of Reduced Frequency . . . . . . . . . . . . . . . . 60

4.3.3 Effect of Amplitude . . . . . . . . . . . . . . . . . . 63

4.4 Comparison to Other Aerodynamic Models . . . . . . . . . . . 79

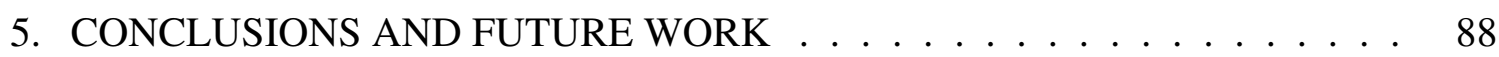

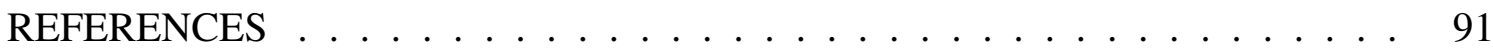




\section{LIST OF FIGURES}

FIGURE

Page

1.1 Vertical-axis wind turbine and horizontal-axis wind turbine. . . . . . . 3

1.2 Typical power curve for horizontal-axis wind turbine. . . . . . . . . . 4

1.3 Relative velocity as seen by a section of the blade at radial location $r$. Lift and drag are shown as well as in-plane resultant force, $F_{i p}$, and out-ofplane resultant force $F_{o p} \ldots \ldots \ldots \ldots$. . . . . . . . . 5

1.4 Sources of uneven loads on rotor and the resulting blade deflections. . . . 6

1.5 Total velocity as seen by different blade sections along the radius of the rotor 7

2.1 Test setup - not drawn to scale. . . . . . . . . . . . . . . . . 11

2.2 First eigenmode with linear fits for the NREL 5MW reference turbine. Dashed lines indicate the portion of the model that would be positioned beneath the wind-tunnel floor. . . . . . . . . . . . . . . . 12

2.3 Blade model installed at Oran Nick's Low Speed Wind Tunnel. Wind is directed into the page. . . . . . . . . . . . . . 16

2.4 Assembled blade. . . . . . . . . . . . . . . . . . 17

2.5 Blade model with top pieces removed. . . . . . . . . . . . . . . 17

2.6 Approximate location of pressure taps on blade section. . . . . . . . . . 17

2.7 Flexible base. . . . . . . . . . . . . . . . . . . . . . 18

2.8 Detail views of bearing blocks that restrict edgewise motion. . . . . . . . 19

2.9 Equivalent mechanical system for flapwise bending of rod . . . . . . . . . 19

2.10 Solid model of the pitch actuation mechanism. The crank wheel has 4 different eccentric holes for varying the amplitude of oscillation . . . . . 20

2.11 Plot of the magnitude of the transfer function for a $1.5 \mathrm{~m}$ tube with an inner diameter of $1.6 \mathrm{~mm} \ldots \ldots \ldots 22$ 
2.12 Bench-top calibration rig for in situ estimation of tubing parameters. . . .

2.13 Example pressure pulse. There is a slight spike on the front end of the pulse due to resonance. . . . . . . . . . . . . . .

2.14 Fourier transform of example pressure pulse. The pulse generates reliable signals up to $50 \mathrm{~Hz}$, above which the signal-to-noise ratio becomes too small. 24

2.15 An example distribution of pressure over the NACA $63_{3}-418$ at $0.5^{\circ}$ angle of attack. . . . . . . . . . . . . . . . . 25

2.16 Diagram of electronics used for data acquisition. . . . . . . . . . 27

3.1 Standard nomenclature for $2 \mathrm{D}$ unsteady airfoil theory. . . . . . . . . . . 30

3.2 Top: lift coefficient distribution along the span at various times for a step change in twist angle. Bottom: integrated lift coefficient over whole blade versus time. . . . . . . . . . . . . . . . . .

3.3 Flap motion for a step change in twist of $1^{\circ}$ for various numbers of even modes used in the simulation, $N \ldots \ldots \ldots \ldots$

3.4 Diagram of the mounting bracket, viewed from downstream and looking upstream. This illustrates why integrated twisting moment cannot be measured in these experiments. The unmeasured $M_{\text {edge }}$ component twists the torsional spring at large angles of flap deflection. . . . . . . . . .

4.1 Measurement of spring constant for flap motion. Note the slight hysteresis caused by plastic deformation. . . . . . . . . . . . . . 42

4.2 Measurement of spring constant for twist motion. Note the slight hysteresis caused by plastic deformation. . . . . . . . . . . . . . 42

4.3 Measurement of strain gauge gain for flap moment. . . . . . . . . . . 43

4.4 Measurement of strain gauge gain for twist moment. . . . . . . . . . . 43

4.5 Impulse response of flap motion, as measured by the strain gauge. The dashed line shows the exponential decay from damping. . . . . . . . .

4.6 Impulse response of twist motion, as measured by the rate gyro. The dashed line shows the exponential decay from damping. . . . . . . . . . 
4.7 Bode plot for 24 inch tube. Below $60 \mathrm{~Hz}$, frequencies are actually amplified instead of attenuated. Transfer function was fit using only data left of the dashed line. . . . . . . . . . . . . . . . . . . . . . .

4.8 Bode plot for 42 inch tube. Transfer function was fit using only data left of the dashed line. . . . . . . . . . . . . . . . . . .

4.9 Bode plot for 60 inch tube. Transfer function was fit using only data left of the dashed line. . . . . . . . . . . . . . . . . . . . . . . . . 48

4.10 Bode plot for 66 inch tube. Transfer function was fit using only data left of the dashed line. . . . . . . . . . . . . . . . . . .

4.11 Bode plot for 78 inch tube. Transfer function was fit using only data left of the dashed line. . . . . . . . . . . . . . . . . . . . . . 50

4.12 Reconstruction of reference signal for 24 inch tube. . . . . . . . . . . 51

4.13 Reconstruction of reference signal for 42 inch tube. . . . . . . . . . 51

4.14 Reconstruction of reference signal for 60 inch tube. . . . . . . . . . . 52

4.15 Reconstruction of reference signal for 66 inch tube. . . . . . . . . . 52

4.16 Reconstruction of reference signal for 78 inch tube. . . . . . . . . 53

4.17 Static angle sweep at $7 \mathrm{~m} / \mathrm{s} \ldots \ldots \ldots \ldots \ldots \ldots \ldots$

4.18 Static angle sweep at $10 \mathrm{~m} / \mathrm{s} . \ldots \ldots \ldots \ldots \ldots \ldots$

4.19 Static angle sweep. . . . . . . . . . . . . . . . . . . 55

4.20 Rate gyroscope measurement of twisting motion for $k_{c}=0.098, V=$ $10 \mathrm{~m} / \mathrm{s}$, and $A_{\phi_{t}}=2^{\circ}$. The dashed lines represent the windows of each period to be averaged. The first 6 of 20 windows are shown here. . . . . . 57

4.21 Each of the 20 windows from Figure 4.20, shown in grey, stacked on top of each other. The phase-lock average is shown in black. . . . . . . . 57

4.22 Twist motion measurements for the baseline case, $k_{c}=0.098, V=10 \mathrm{~m} / \mathrm{s}$, and $A_{\phi_{t}}=2^{\circ}$. The total twist is used as the input to the simulation model. 
4.23 Simulated flap response and moment for the baseline case, $k_{c}=0.098$, $V=10 \mathrm{~m} / \mathrm{s}$, and $A_{\phi_{t}}=2^{\circ}$, compared to those measured by the experiment. The measured twist motion, used as input to the simulation model, is shown at the bottom. . . . . . . . . . . . . . 59

4.24 Center-span lift coefficient for the baseline case, $k_{c}=0.098, V=10 \mathrm{~m} / \mathrm{s}$, and $A_{\phi_{t}}=2^{\circ} \ldots \ldots \ldots \ldots \ldots \ldots \ldots$

4.25 Simulated lift coefficients at various span locations for the baseline case, $k_{c}=0.098, V=10 \mathrm{~m} / \mathrm{s}$, and $A_{\phi_{t}}=2^{\circ}$. . . . . . . . . . .

4.26 Simulated twist moment for the baseline case, $k_{c}=0.098, V=10 \mathrm{~m} / \mathrm{s}$, and $A_{\phi_{t}}=2^{\circ}$, compared to that measured by the experiment. . . . . . . 62

4.27 Simulated flap moment and motion for $k_{c}=0.13, V=10 \mathrm{~m} / \mathrm{s}$, and $A_{\phi_{t}}=2^{\circ} .64$

4.28 Simulated flap moment and motion for $k_{c}=0.22, V=10 \mathrm{~m} / \mathrm{s}$, and $A_{\phi_{t}}=2^{\circ} .65$

4.29 Simulated flap moment and motion for $k_{c}=0.31, V=10 \mathrm{~m} / \mathrm{s}$, and $A_{\phi_{t}}=2^{\circ} .66$

4.30 Simulated flap moment and motion for $k_{c}=0.39, V=10 \mathrm{~m} / \mathrm{s}$, and $A_{\phi_{t}}=2^{\circ} .67$

4.31 Center-span lift coefficient for $k_{c}=0.22, V=10 \mathrm{~m} / \mathrm{s}$, and $A_{\phi_{t}}=2^{\circ} \ldots . \quad 68$

4.32 Center-span lift coefficient for $k_{c}=0.31, V=10 \mathrm{~m} / \mathrm{s}$, and $A_{\phi_{t}}=2^{\circ} \ldots . \quad 68$

4.33 Center-span lift coefficient for $k_{c}=0.39, V=10 \mathrm{~m} / \mathrm{s}$, and $A_{\phi_{t}}=2^{\circ} \ldots \ldots 9$

4.34 Center-span lift coefficient for $k_{c}=0.56, V=7 \mathrm{~m} / \mathrm{s}$, and $A_{\phi_{t}}=2^{\circ}$. The lower velocity is used to achieve a higher $k$ value at the maximum frequency of the motor. . . . . . . . . . . . . . 69

4.35 Simulated lift coefficients at various span locations for $k_{c}=0.22, V=$ $10 \mathrm{~m} / \mathrm{s}$, and $A_{\phi_{t}}=2^{\circ} \ldots \ldots \ldots \ldots \ldots$. . . . . . . . 70

4.36 Simulated lift coefficients at various span locations for $k_{c}=0.31, V=$ $10 \mathrm{~m} / \mathrm{s}$, and $A_{\phi_{t}}=2^{\circ} \ldots \ldots \ldots \ldots \ldots$

4.37 Simulated lift coefficients at various span locations for $k_{c}=0.39, V=$ $10 \mathrm{~m} / \mathrm{s}$, and $A_{\phi_{t}}=2^{\circ} \ldots \ldots \ldots \ldots \ldots \ldots$. . . . . . . . . 72

4.38 Simulated lift coefficients at various span locations for $k_{c}=0.56, V=$ $7 \mathrm{~m} / \mathrm{s}$, and $A_{\phi_{t}}=2^{\circ} \ldots \ldots \ldots \ldots \ldots$

4.39 Simulated flap moment and motion for $k_{c}=0.22, V=10 \mathrm{~m} / \mathrm{s}$, and $A_{\phi_{t}}=1^{\circ} .74$ 
4.40 Simulated flap moment and motion for $k_{c}=0.22, V=10 \mathrm{~m} / \mathrm{s}$, and $A_{\phi_{t}}=4^{\circ} 75$

4.41 Simulated lift coefficients at the center-span $k_{c}=0.22, V=10 \mathrm{~m} / \mathrm{s}$, and

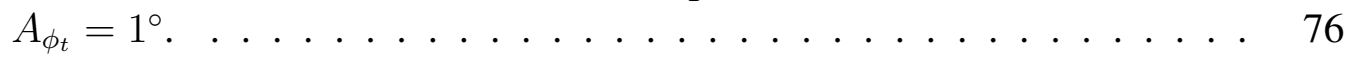

4.42 Simulated lift coefficients at the center-span $k_{c}=0.22, V=10 \mathrm{~m} / \mathrm{s}$, and $A_{\phi_{t}}=4^{\circ} \ldots \ldots \ldots \ldots \ldots \ldots \ldots \ldots \ldots \ldots \ldots \ldots \ldots \ldots \ldots \ldots \ldots$

4.43 Simulated lift coefficients at various span locations for $k_{c}=0.22, V=$ $10 \mathrm{~m} / \mathrm{s}$, and $A_{\phi_{t}}=1^{\circ} \ldots \ldots \ldots \ldots$. . . . . . . . . 77

4.44 Simulated lift coefficients at various span locations for $k_{c}=0.22, V=$ $10 \mathrm{~m} / \mathrm{s}$, and $A_{\phi_{t}}=4^{\circ} \ldots \ldots \ldots \ldots \ldots \ldots$

4.45 Moment and motion for $k_{c}=0.098, V=10 \mathrm{~m} / \mathrm{s}$, and $A_{\phi_{t}}=2^{\circ}$ using 3 different aerodynamic models. . . . . . . . . . . . . . 80

4.46 Moment and motion for $k_{c}=0.22, V=10 \mathrm{~m} / \mathrm{s}$, and $A_{\phi_{t}}=2^{\circ}$ using 3 different aerodynamic models. . . . . . . . . . . 81

4.47 Moment and motion for $k_{c}=0.39, V=10 \mathrm{~m} / \mathrm{s}$, and $A_{\phi_{t}}=2^{\circ}$ using 3 different aerodynamic models. . . . . . . . . . . 82

4.48 Center-span sectional lift for $k=0.098, V=10 \mathrm{~m} / \mathrm{s}$, and $A_{\phi_{t}}=2^{\circ}$ using 3 different aerodynamic models. . . . . . . . . . . . .

4.49 Center-span sectional lift for $k=0.22, V=10 \mathrm{~m} / \mathrm{s}$, and $A_{\phi_{t}}=2^{\circ}$ using 3

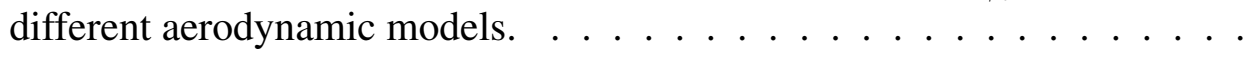

4.50 Center-span sectional lift for $k=0.39, V=10 \mathrm{~m} / \mathrm{s}$, and $A_{\phi_{t}}=2^{\circ}$ using 3 different aerodynamic models. . . . . . . . . . . . . 84

4.51 Sectional lift at various spanwise locations for $k_{c}=0.098, V=10 \mathrm{~m} / \mathrm{s}$, and $A_{\phi_{t}}=2^{\circ}$ using 3 different aerodynamic models. . . . . . . . . .

4.52 Sectional lift at various spanwise locations for $k_{c}=0.22, V=10 \mathrm{~m} / \mathrm{s}$, and $A_{\phi_{t}}=2^{\circ}$ using 3 different aerodynamic models. . . . . . . . . . 86

4.53 Sectional lift at various spanwise locations for $k_{c}=0.39, V=10 \mathrm{~m} / \mathrm{s}$, and $A_{\phi_{t}}=2^{\circ}$ using 3 different aerodynamic models. . . . . . . . . . . . 


\section{INTRODUCTION}

The past decades have seen a trend of progressively larger wind turbines. This trend is expected to accelerate as the industry shifts to offshore wind installations. The power produced by a turbine scales with its diameter squared, but the material cost scales with weight, thus theoretically scaling by diameter cubed. However, the historical trend has seen blade weight scale with diameter to the power of 2.3 as each generation of larger turbines reduces the equivalent structural weight of the blades to remain economically viable [1]. This trend of longer, more flexible blades, combined with large unsteady aerodynamic loads involved in wind turbine operation, can lead to early failure of components due to fatigue.

Designers use a variety of simulation tools to predict blade motion and fatigue life of a turbine. Accurate prediction of unsteady loads would reduce the need for overly conservative factors of safety. This, in turn, would lead to lighter, cheaper blades and would provide estimates of performance and blade life, allowing turbine operators to better predict the return on their investment. Dynamic control is also being proposed to reduce the weight and cost of turbines by actively alleviating unsteady loads [2]. Both these techniques rely on solid understanding of the coupling between unsteady aerodynamics and structural dynamics, or aeroelasticity.

Though many computational tools exist for modeling aeroelasticity in wind turbines, very few experiments have been done to validate them. Existing experiments for aeroelasticity focus primarily on conditions relevant to aircraft. Aircraft mainly operate in steady flow and fatigue arises from insufficient damping of vibration modes, so designers are concerned with stability analysis. Wind turbines on the other hand, operate in extremely unsteady flow conditions, but are stable, so the main concern is stiffness and load allevi- 
ation. The most common area of study for aircraft aeroelasticity is the flutter instability, which arises when the damping of a vibration mode becomes negative causing vibrations to grow until failure or until non-linear structural stiffness keeps the oscillation within certain bounds-called limit cycle oscillations (LCO). These phenomenon are not yet a problem in normal operation of today's turbines. However, this may change as blades become longer and less stiff in torsion [3].

The most frequently cited validation experiment for wind turbines was conducted by the National Renewable Energy Lab (NREL) in 2000. The experiment consisted of a series of wind tunnel tests on a 10-m-diameter wind turbine in the NASA-Ames $80 \mathrm{ft}$ by $120 \mathrm{ft}$ wind tunnel [4]. The data was used to perform a blind validation of various wind turbine simulation codes. These tests provided much needed experimental data for unsteady aerodynamics and demonstrated just how inaccurate all simulations were at the time. However, this turbine was specifically designed with stiff blades that minimized aeroelastic phenomenon [5]. Although there is a clear need for such studies, there has been a lack of wind-tunnel tests to validate aeroelastic models for highly flexible wind turbine blades. This dissertation aims to fill this gap with a series of tests on a compliant test platform at the Texas A\&M Low Speed Wind Tunnel (LSWT).

\subsection{Wind Turbine Aerodynamics}

The two main types of wind turbines, vertical axis and horizontal axis, are shown in Figure 1.1. Vertical-axis wind turbines (VAWTs) have the advantage of being omnidirectional, and the heavy generator can be placed on the ground, reducing the cost of the structure. Horizontal-axis wind turbines (HAWTs) are more efficient, and the rotor can be placed atop a tower, where the wind is stronger. Due to their advantages, HAWTs make up the majority of large-scale wind turbine installations [6]. As such, this work focuses solely on the aerodynamics of HAWTs. 


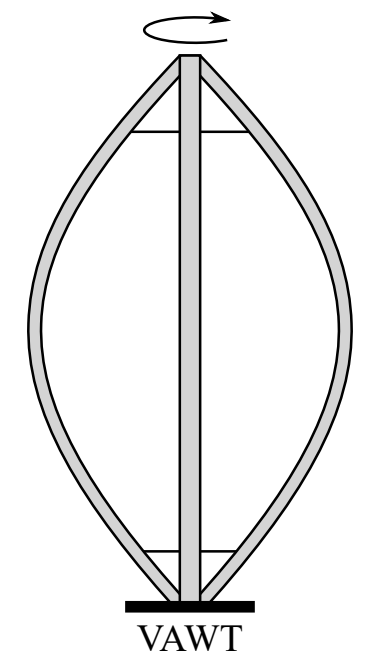

VAWT

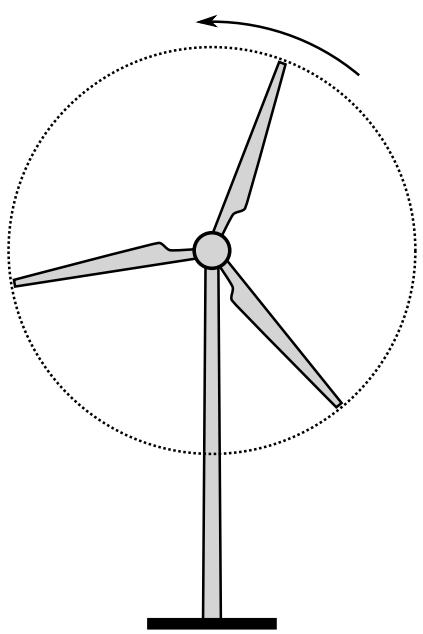

HAWT

Figure 1.1: Vertical-axis wind turbine and horizontal-axis wind turbine.

Wind turbines extract energy from air passing through the swept area of the blades. For a uniform flow field of velocity $V_{w}$, the power flux through this plane is given by $\rho_{\text {air }} A V_{w}^{3} / 2$, where $\rho$ is the air density and $A$ is the area of the rotor disk. The coefficient of power of a wind turbine is the fraction of this power flux that is actually extracted. This coefficient of power has an upper limit of 0.593, known as Betz Law. A typical turbine can achieve about $80 \%$ of this limit [2]. The power output of a turbine as a function of wind speed, shown in Figure 1.2, has four distinct regions. In Region I, the wind is too slow to generate enough torque to overcome friction and reliably turn the rotor. Rotors are deliberately parked at these wind speeds. In Region II, the power output follows a cubic function of wind speed. Region III begins once the power available to the turbine reaches the maximum rating of the generator. Above this speed, blade pitch is reduced to maintain rated generator power at fixed RPM. In Region IV, operation is too dangerous; the rotor is shut down using brakes, and the blades are feathered to prevent damage.

Figure 1.3 shows the relative velocity as seen by a blade section of a al turbine operating in Region II. In this region, the turbine operates with a constant tip-speed ratio, 


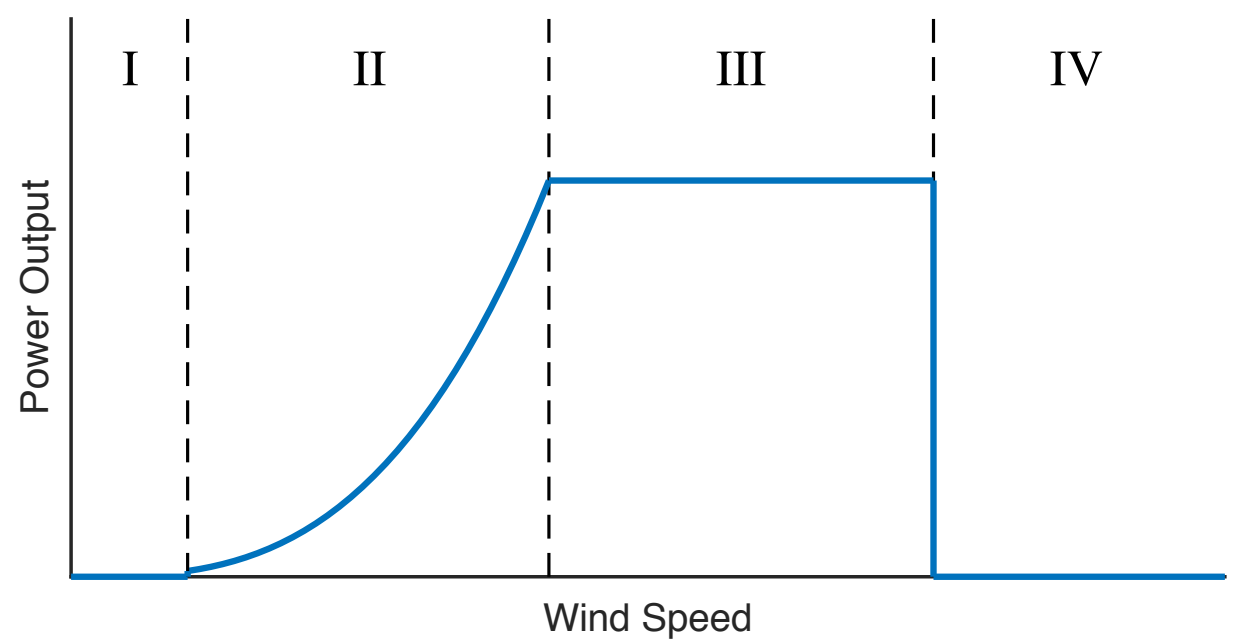

Figure 1.2: Typical power curve for horizontal-axis wind turbine.

$\lambda=R \Omega / V_{w}$, where $R$ is the rotor radius, $\Omega$ is the angular velocity of the rotor, and $V_{w}$ is the wind velocity. The optimal tip speed ratio is dependent on the design of the turbine, but a value around 7 is typical [2]. This means that the velocity seen by the outboard half of the blade is dominated by the rotation component of the velocity. The resultant force from the lift, $L$, and drag, $D$, is transformed into a component aligned with the rotor plane, $F_{i p}$ and an out-of-plane component $F_{o p}$. The in-plane force is responsible for providing the torque that generates power and also causes some bending of the blade in the "edgewise" direction. The out-of-plane force does not contribute anything to power generation, but makes up a majority of the load borne by the blade, hub, and tower structures. This force contributes to "flapwise" bending of the blade.

\subsection{Fatigue}

Wind turbine blade sections experience unsteady relative velocity and angle of attack caused by atmospheric turbulence, blade movement through the atmospheric boundary layer, yaw misalignment, flow interaction with the tower, and the wakes of upstream tur- 


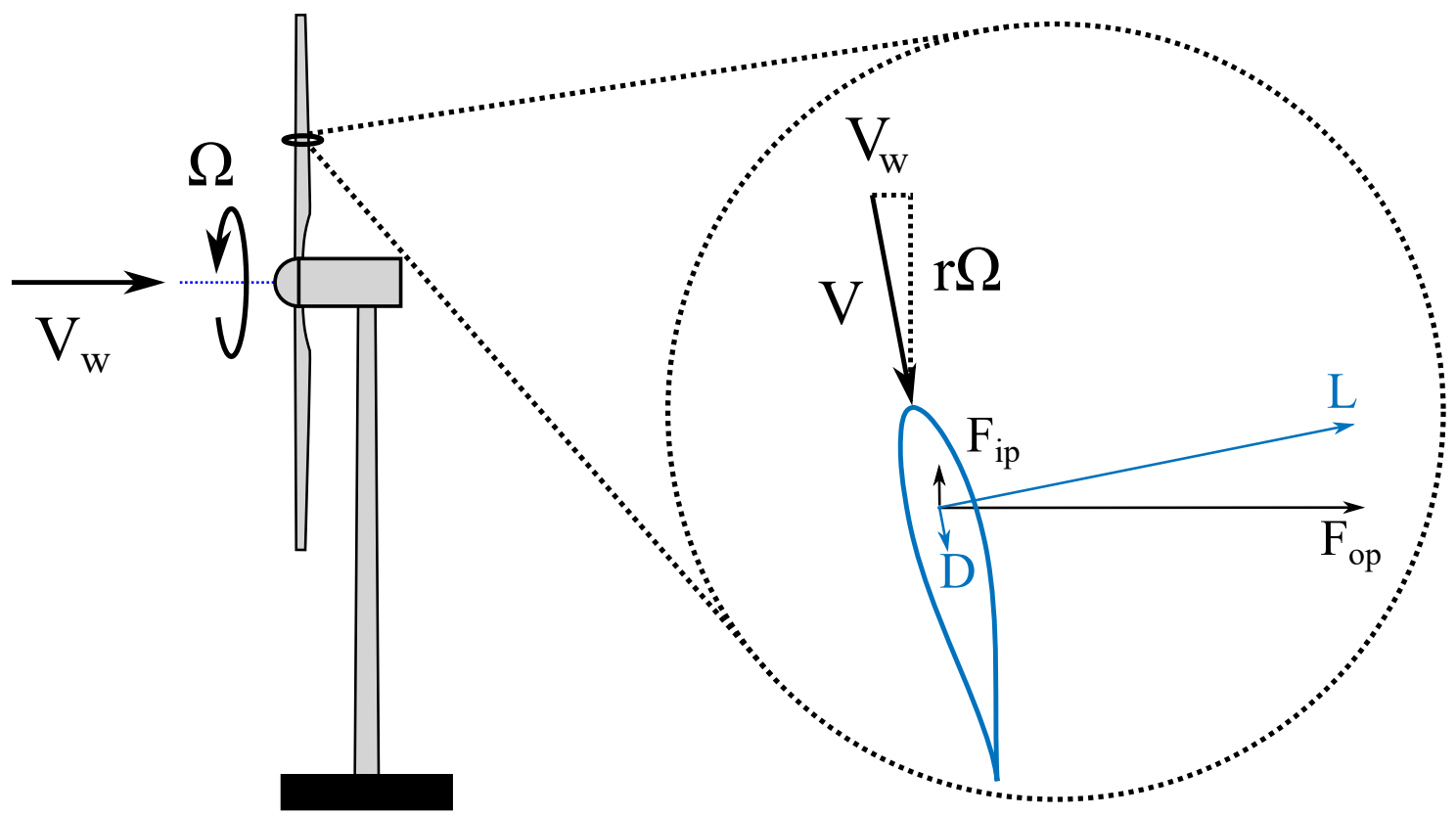

Figure 1.3: Relative velocity as seen by a section of the blade at radial location $r$. Lift and drag are shown as well as in-plane resultant force, $F_{i p}$, and out-of-plane resultant force $F_{\text {op }}$.

bines. These factors result in unsteady aerodynamic loads that lead to undesirable blade and tower fatigue that reduces turbine lifetime. The unsteady load sources and resulting motions are summarized in Figure 1.4. These load cycles, combined with the desire for long operational lifetimes, make fatigue a principal design concern for wind turbine rotors.

The primary source of varying loads through the revolution is the atmospheric boundary layer. The wind speed is not constant as a function of altitude, but typically increases at higher altitudes. In addition, the wind direction can change substantially as a function of altitude. For the outboard half of the blade (which is responsible for $75 \%$ of the power generated), the velocity is dominated by the rotation of the blade (as shown in Figure 1.5), so changes in wind speed have little effect on the dynamic pressure. However, the variable inflow does have a substantial impact on the angle-of-attack, which affects sectional lift production and the loads experienced by the blade. Yaw misalignment has a similar effect; 
when the wind is not perpendicular to the rotor disk, the blade will be moving downwind at the top of its path and advancing upwind at the bottom, or vice versa. This can also be visualized by rotating the $V_{w}$ vector in Figure 1.5. The controller responsible for keeping the rotor aligned with the wind is slow, and the sensor mounted on the nacelle is susceptible to bias error due to the being placed downstream of the rotor, making yaw misalignment a common issue for wind turbines [7]. As with the atmospheric boundary layer, this is seen mostly as an angle-of-attack fluctuation.

In addition to the once-per-revolution loads experienced in normal operation, wind turbines experience unexpected gusts or extreme weather events. As the industry shifts toward offshore wind power, it is becoming even more important to understand how the wind turbine will respond to extreme gusts during operation or with blades parked as they would be during extreme weather.
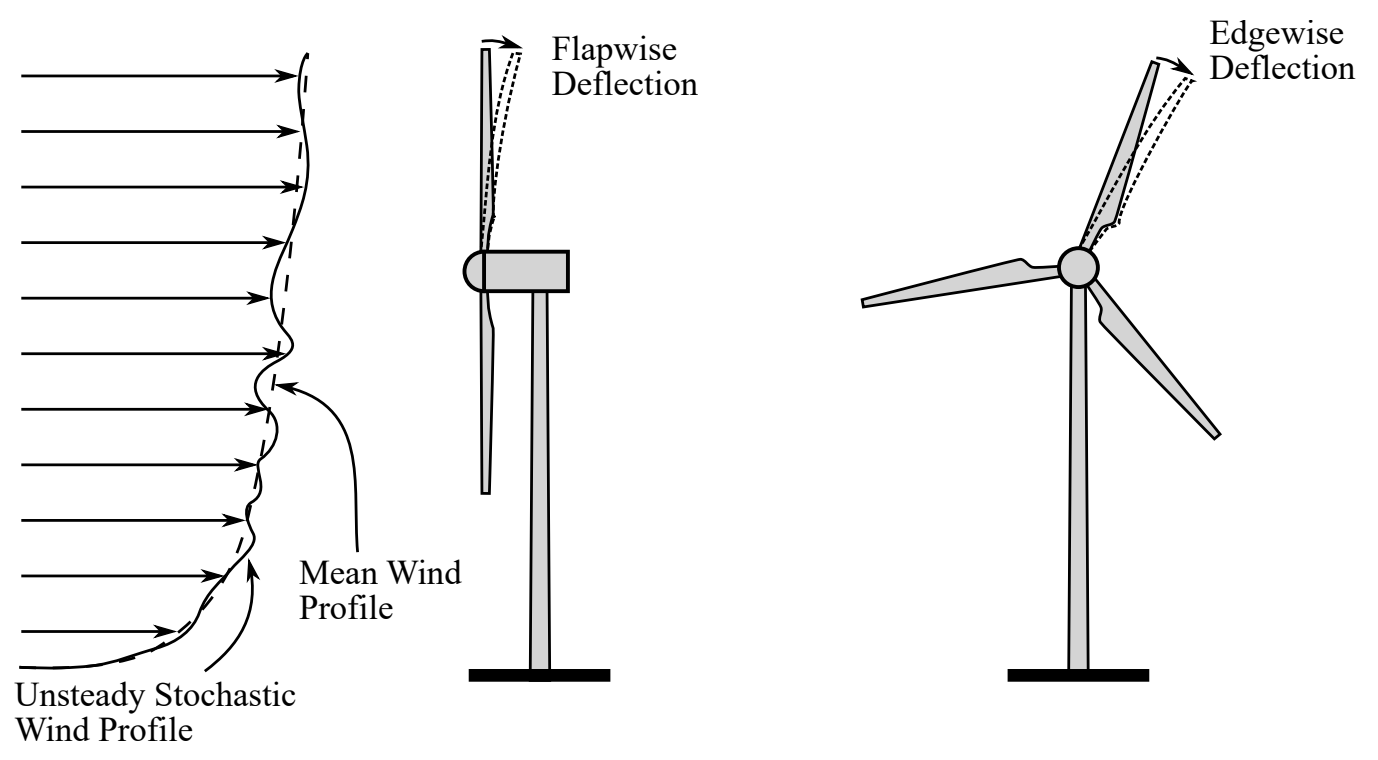

Figure 1.4: Sources of uneven loads on rotor and the resulting blade deflections. 


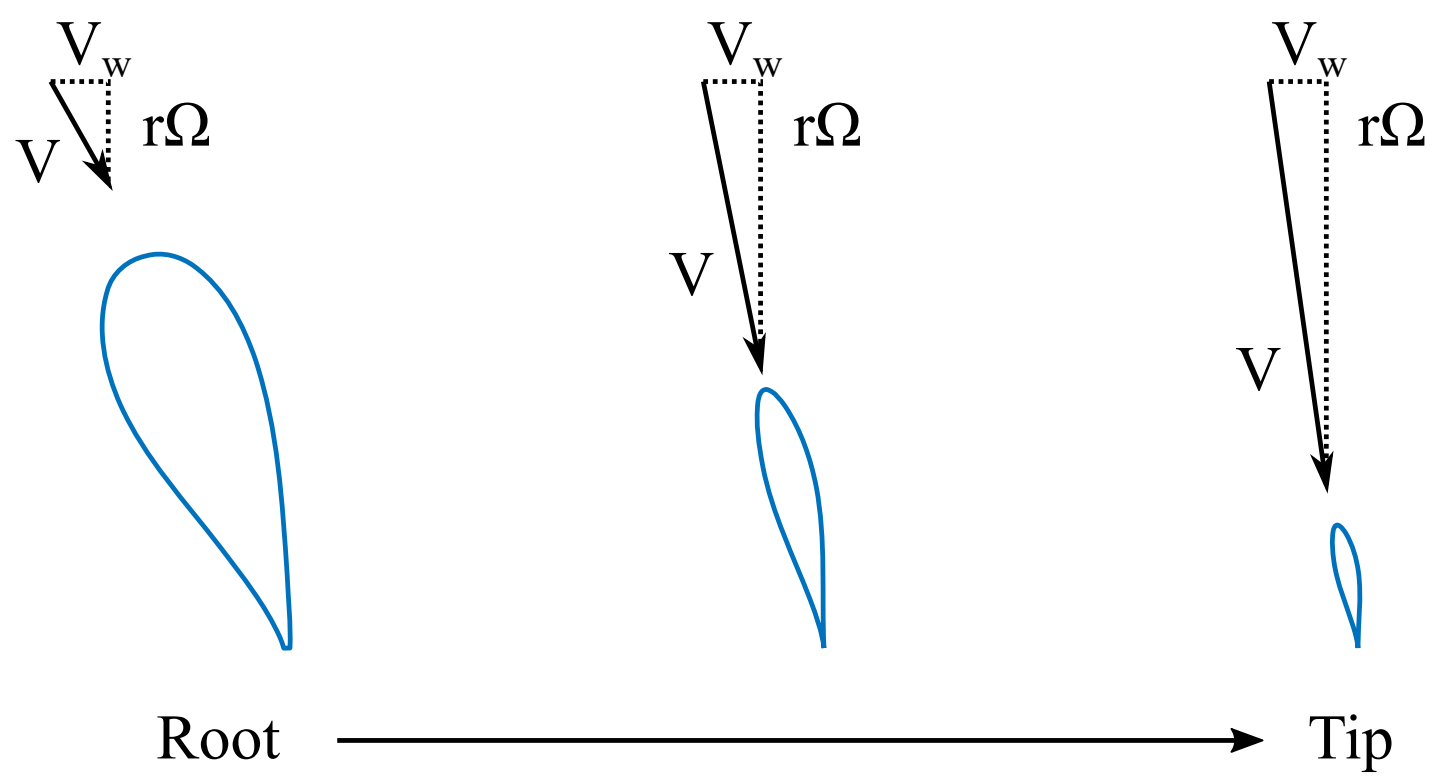

Figure 1.5: Total velocity as seen by different blade sections along the radius of the rotor

\subsection{Objectives}

This project has three principal objectives:

1. Design and build a wind-tunnel test article, aeroelastically similar to a large-scale wind turbine blade, for validation of aeroelastic models.

2. Develop a time-domain aeroelastic model for simulating the response of the blade to various disturbances.

3. Compare measured aerodynamic loads to those predicted by different unsteady aerodynamic models.

The first objective is to build a flexible model with elasticity and mass parameters tuned to match the response of a full-scale blade. The model motion is driven by elastic response to aerodynamic disturbances, instead of driven to a prescribed motion using motors. The test model is tapered and cantilevered, instead of a full-span 2D airfoil. Previous tests have 
investigated dynamic 2D airfoil sections. The work expands on this foundation to provide the 3D aerodynamics important for wind turbine blades. The blade will also serve as a test platform for future aeroelastic tests and possible active control experiments.

The second objective is to develop a predictive model for the test article. This model must be time domain, as not all disturbances are periodic. Transient disturbances such as gusts are important. A time domain model is also needed for active control loops in future tests. Additionally, the model must capture the 3D aerodynamics introduced by the tapered, finite-span blade. The model will take prescribed aerodynamic disturbances as inputs and will output resulting blade loads and motion.

The third objective is to compare these simulated loads and motions to those measured experimentally in wind tunnel tests. These tests serve to validate predictive models, demonstrate where they break down, and highlight necessary extensions to the models. Such validation is necessary to research concerning material fatigue and load alleviation. The research presented here is mainly an aerodynamics study, and does not directly address fatigue or load alleviation, but rather serves as an important first step for future work in these areas. 


\section{EXPERIMENT DESIGN}

The first objective of this work is to develop a test platform for aeroelastic wind tunnel experiments. Babbar [8] presents an aeroelastic wind tunnel test platform from which this work is partially derived. Babbar's test platform consists of a 2D airfoil spanning a 3 foot by 4 foot wind tunnel mounted to an adjustable non-linear spring mounting system with pitch and plunge degrees of freedom. These experiments were primarily concerned with validating a predictive model for the onset of flutter and limit-cycle oscillations for non-linear structures. The experiment also featured a second airfoil mounted upstream and driven in pitch and plunge to generate gusts that travel downstream to the elastically mounted airfoil. This was used to validate a model for predicting the resulting loads and motion in response to a gust. Instead of a 2D airfoil spanning the test section, this work features a tapered, cantilevered blade. This will allow for validation of $3 \mathrm{D}$ extensions of unsteady aerodynamic models that include blade twist and flapping motion.

Model validation was performed through a series of wind tunnel tests on a simplified elastic blade. Tests were conducted in the Texas A\&M Low Speed Wind Tunnel (LSWT). The LSWT is a closed-loop tunnel with a 7 foot by 10 foot test section and a top speed of $90 \mathrm{~m} / \mathrm{s}$. The outputs for these experiments are the unsteady blade motion and aerodynamic loads. Design of a properly scaled dynamic aeroelastic experiment is a significant challenge. With the importance of the coupling between the air and the structure, it is not sufficient to merely match Reynolds number. Careful consideration of the equations of motion is needed to capture the relevant physics, and it will not be possible to perfectly match all parameters and degrees of freedom of the full blade. This chapter describes the design process for the test article, and the experimental methods employed in these tests.

The experimental platform is modeled after the NREL $5 \mathrm{MW}$ reference turbine [9]. To 
simplify the design, a two-degree-of-freedom model that captures out-of-plane flapping and torsional motions of the outboard $35 \%$ of the blade will be used. This outboard region of the blade sees the highest velocities and largest angle-of-attack fluctuations induced by blade motion. The outboard end of the blade is also subject to strongly 3D aerodynamics generated by the tip vortex. The outboard blade is modeled using a rigid section attached to a compliant base, shown in Figure 2.1. Designing a fully flexible blade was deemed impractical and unnecessary for this test. The displacements caused by the bending of the outer $35 \%$ of the blade are negligible compared to those caused by deflection of the inboard $65 \%$. This can be seen in Figure 2.2. Using a compliant base also allows for adjusting the flexibility for future experiments; a fully compliant blade would require an entirely new blade to be constructed. The compliant base is mounted below the wind tunnel floor and has two degrees of freedom: flapping and torsion. The flapping degree of freedom is the most compliant bending mode and has the largest forces applied in its direction. The twist degree of freedom is more rigid compared to its smaller forcing, but twist has large effect on lift and is critical to include.

\subsection{Model Reduction}

The first step in approximating the blade as a rigid section is an eigenmode analysis of the flapping motion of the blade. A linear regression was applied to the outer portion of the first out-of-plane flapping mode, the lowest-frequency structural mode. Figure 2.2 shows three linear regressions applied to various portions of the outboard end of the blade. The $35 \%$ line was chosen as the largest fit with acceptable linearity. This line also has the advantage of minimal out-of-plane motion at its starting radius. This minimizes the amount of structure that must be placed under the floor. 


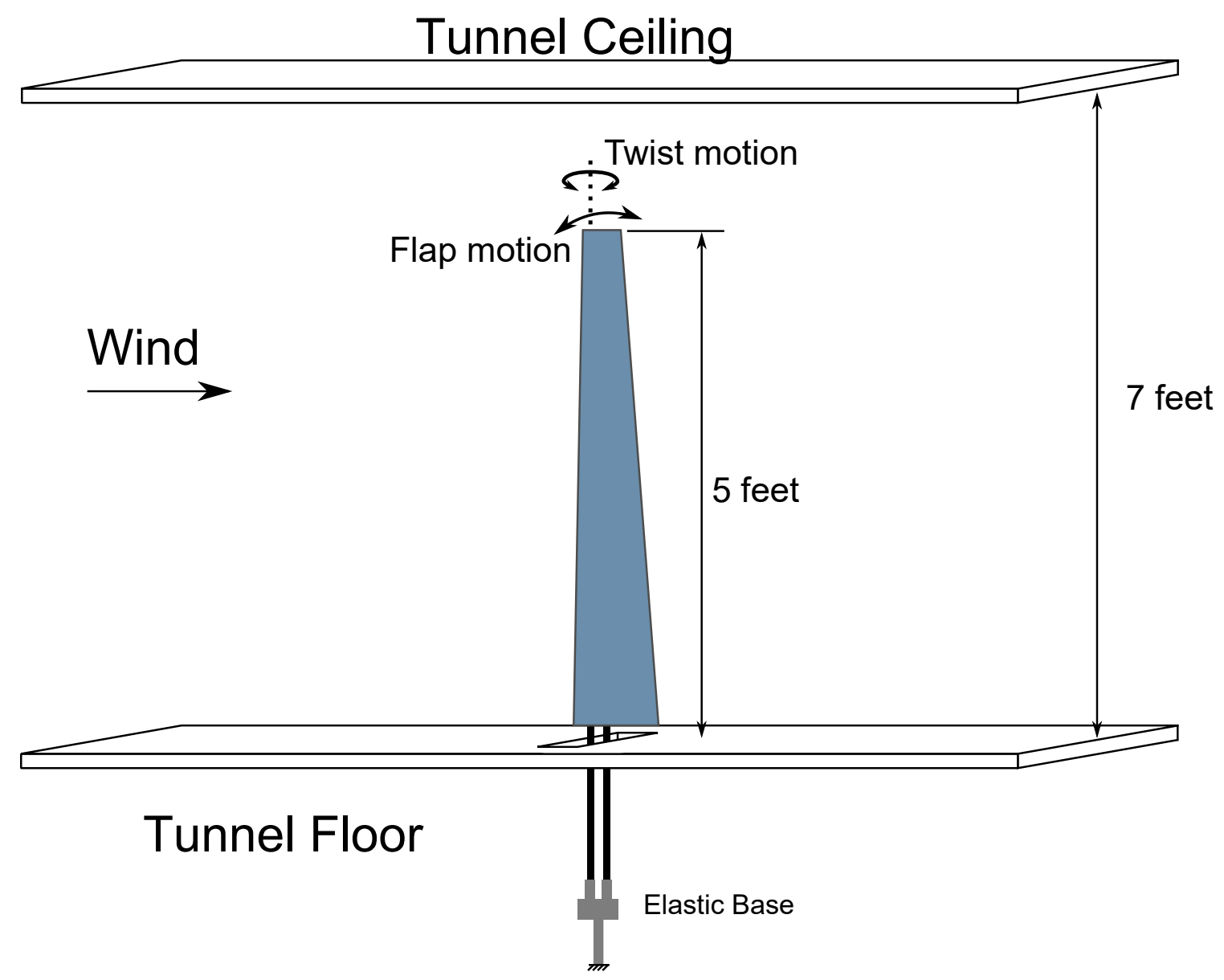

Figure 2.1: Test setup—not drawn to scale. 


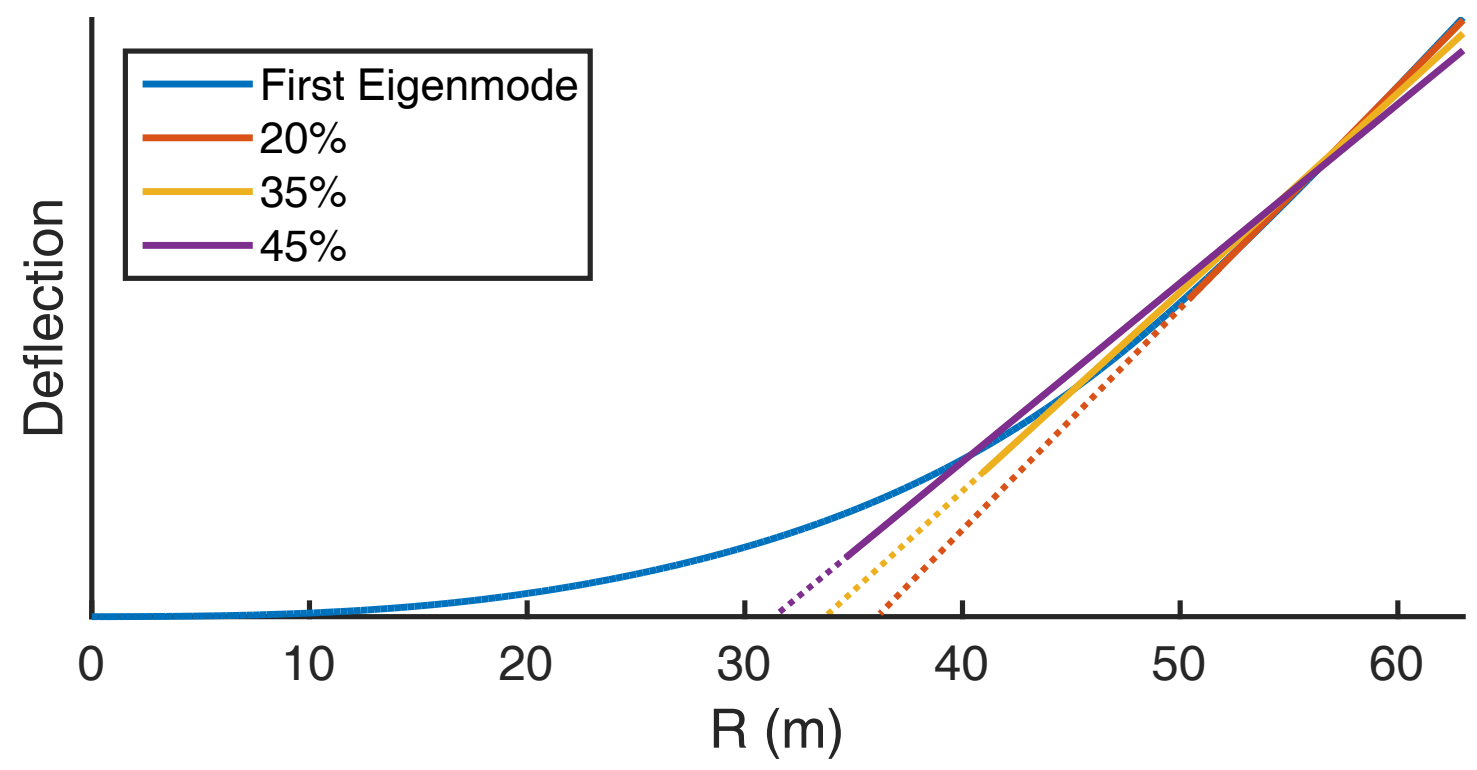

Figure 2.2: First eigenmode with linear fits for the NREL 5MW reference turbine. Dashed lines indicate the portion of the model that would be positioned beneath the wind-tunnel floor.

\subsection{Dynamic Aeroelastic Scaling}

The coupling between the aerodynamics and structural dynamics introduces non-dimensional parameters that must match between the full-scale and reduced-scale model. This section follows the process outlined by Dugundji [10]. The Navier-Stokes equations for incompressible viscous flow around an elastic body are given below using Einstein's index summation notation.

$$
\begin{gathered}
\frac{\partial v_{k}}{\partial x_{k}}=0 \\
\rho_{\text {air }}\left[\frac{\partial v_{i}}{\partial t}+v_{k} \frac{\partial v_{i}}{\partial x_{k}}\right]=-\frac{\partial p}{\partial x_{i}}+\mu \frac{\partial^{2} v_{i}}{\partial x_{k} \partial x_{k}}
\end{gathered}
$$

where $v_{i}$ is the local velocity vector, $p$ is local pressure, $\rho_{\text {air }}$ is air density, and $\mu$ is the kinematic viscosity of air. The following boundary condition applies at the surface of the 
body:

$$
v_{i}=\frac{\partial u_{i}}{\partial t}
$$

where $u_{i}$ is the local displacement vector at the surface of the body. This states that the velocity of the structural displacement of the surface is equal to the fluid velocity at the surface. Non-dimensional variables are introduced.

$$
\bar{x}_{i}=x_{i} / R, \quad \bar{t}=t / t_{0}, \quad \bar{p}=p / \rho_{a i r} V^{2}, \quad \bar{v}_{i}=v_{i} / V, \quad \bar{u}_{i}=u_{i} / u_{0}
$$

where $R$ is the span of the blade, $V$ is freestream velocity, $t_{0}$ is an arbitrary reference time, and $u_{0}$ is an arbitrary reference displacement. Rewriting equations $2.1,2.2$, and 2.3 in terms of the non-dimensional variables gives

$$
\begin{gathered}
\frac{\partial \bar{v}_{k}}{\partial \bar{x}_{k}}=0 \\
\left(\frac{R}{V t_{0}}\right) \frac{\partial \bar{v}_{i}}{\partial \bar{t}}+\bar{v}_{k} \frac{\partial \bar{v}_{i}}{\partial \bar{x}_{k}}=-\frac{\partial \bar{p}}{\partial \bar{x}_{i}}+\left(\frac{\mu}{\rho_{\infty} V R}\right) \frac{\partial^{2} \bar{u}_{i}}{\partial \bar{x}_{k} \partial \bar{x}_{k}} \\
\bar{v}_{i}=\left(\frac{u_{0}}{R}\right)\left(\frac{R}{V t_{0}}\right) \frac{\partial \bar{u}_{i}}{\partial \bar{t}}
\end{gathered}
$$

From this, it is clear that, if the following non-dimensional parameters match between the full-sized system and its geometrically scaled model, then the above equations would give identical solutions for the unknown $\bar{p}$ and $\bar{v}_{i}$ :

$$
\frac{\rho_{a i r} V R}{\mu}, \quad \bar{u}_{i}, \quad \frac{V t_{0}}{R}, \quad \frac{u_{0}}{R}
$$

The first term is the Reynolds number, and the second term requires the deflections to be similar. The last two terms serve to define appropriate reference time, $t_{0}$, and reference displacement $u_{0}$. Because $t_{0}$ and $u_{0}$ are arbitrary, they can be chosen to such that their 
respective terms in equation 2.8 are unity: $t_{0}=R / V$ and $u_{0}=R$.

The dynamic stress-strain equations for an elastic, isotropic material are given by

$$
\begin{gathered}
\frac{\partial \sigma_{i k}}{\partial x_{k}}=\rho_{B} \frac{\partial^{2} u_{i}}{\partial t^{2}}=\rho_{B} \frac{\partial v_{i}}{\partial t} \\
\frac{1}{2}\left(\frac{\partial u_{i}}{\partial x_{j}}+\frac{\partial u_{j}}{\partial x_{i}}\right)+\frac{1}{2}\left(\frac{\partial u_{k}}{\partial x_{i}} \frac{\partial u_{k}}{\partial x_{j}}\right)=\frac{1+\nu}{E} \sigma_{i j}-\frac{\nu}{E} \delta_{i j} \sigma_{k k}
\end{gathered}
$$

where $\sigma_{i k}$ is the local strain tensor, $\rho_{B}$ is the local density of the material, $E$ is the local Young's modulus, and $\nu$ is the Poisson's ratio of the material. Because displacement $u_{i}$ can be measured from any arbitrary reference position, $\sigma_{i j}$ and $u_{i}$ are defined to be zero when the air and blade are at rest. At the surface of the body, the stress at the surface is subject to the boundary condition

$$
\sigma_{i j} n_{i} n_{j}=p
$$

where $n_{i}$ is the unit vector normal to the surface at that location and $p$ is a suitable gauge pressure. Introducing the non-dimensional quantities, in addition to the ones introduced earlier,

$$
\bar{\sigma}_{i j}=\frac{\sigma_{i j}}{\sigma_{0}}, \quad \bar{E}=\frac{E}{E_{0}}, \quad \bar{\rho}_{B}=\frac{\rho_{B}}{\rho_{B_{0}}}
$$

If equations $2.9-2.11$ rewritten in terms of the non-dimensional variables, the following non-dimensional parameters emerge:

$$
\frac{E_{0}}{\rho_{\text {air }} V^{2}}, \quad \frac{\rho_{B_{0}}}{\rho_{\text {air }}}, \quad \frac{\sigma_{0}}{\rho_{\text {air }} V^{2}}, \quad \nu, \quad \bar{E}, \quad \bar{\rho}_{B}
$$

The first term relates the stiffness of the blade to the dynamic pressure, the second term states that the densities of the model must match the density of the full-scale blade, the third term defines the arbitrary reference $\sigma_{0}$, and the last three terms require structural 
similarity.

For the purpose of the proposed reduced-order wind-tunnel model, it is useful to recast the parameters in equations 2.8 and 2.13 into lumped mass and spring constants: $m, K_{\text {flap }}$, and $K_{\text {twist }}$. To obtain suitable constants the following relations are used:

$$
\begin{aligned}
K_{\text {flap }} & \propto E_{0} R^{3} \\
K_{\text {twist }} & \propto \frac{E_{0} R^{3}}{2(1+\nu)} \propto K_{\text {flap }} \\
\rho_{\text {air }} & \propto \rho_{B_{0}} \propto \frac{m}{R^{3}} \\
\bar{c} & \propto R
\end{aligned}
$$

The mean aerodynamic chord is denoted as $\bar{c}$. Substituting these into equations 2.8 and 2.13 yields

$$
\frac{\rho_{\text {air }} V \bar{c}}{\mu}, \quad \frac{m V^{2}}{K_{\text {flap }}}, \quad \frac{m}{\rho_{\text {air }} R^{3}}
$$

The first term is the chord-based Reynolds number. The second term requires that the non-dimensional natural frequencies of the model blade and full-scale blade match. The third term requires the average density of the blade to match (the density of air will be the same in both cases). The third term presents the largest design challenge for this project, as wind-turbine blades are quite light for their size.

For the full-scale turbine blade operating at rated conditions (wind speed of $12 \mathrm{~m} / \mathrm{s}$ and spinning at $12.1 \mathrm{rpm}$ ) the average relative flow velocity along the outer $35 \%$ of the blade is $67 \mathrm{~m} / \mathrm{s}$. With the LSWT's top speed of $90 \mathrm{~m} / \mathrm{s}$, it is clearly impossible to match Reynolds number because the chord length must be much smaller than a real blade. Instead, the tunnel operates at the fastest acceptable speed without risk of overloading the blade model. Assuming a span of $1.5 \mathrm{~m}$ and a tunnel speed of $10 \mathrm{~m} / \mathrm{s}$, the mass of the blade and the three spring constants can be calculated: $m, 0.86 \mathrm{~kg} ; K_{\text {flap }}, 88.2 \mathrm{~N} \cdot \mathrm{m}$; and $K_{\text {twist }}, 21.6 \mathrm{~N} \cdot \mathrm{m}$. 


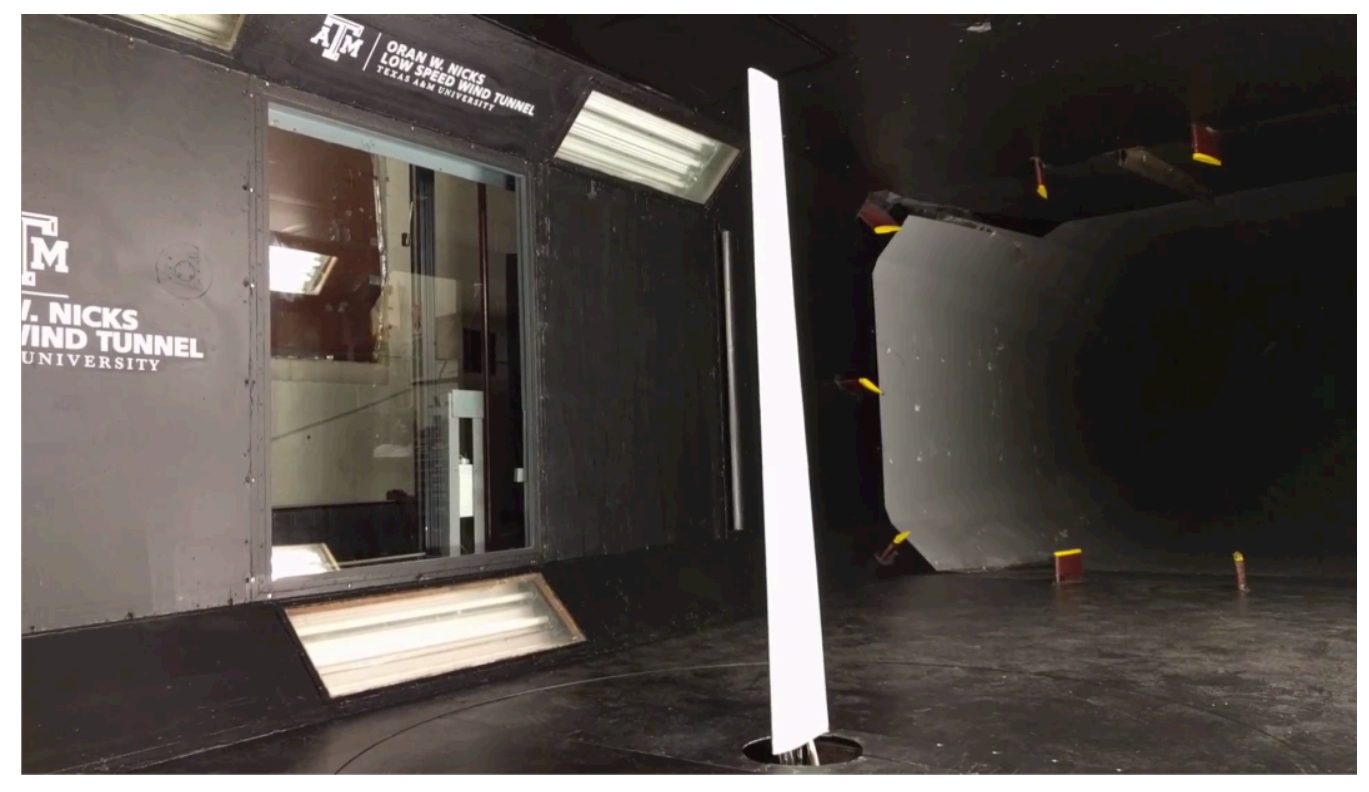

Figure 2.3: Blade model installed at Oran Nick's Low Speed Wind Tunnel. Wind is directed into the page.

The natural frequency of the flap mode is $1.4 \mathrm{~Hz}$.

\subsection{Rigid Blade}

To achieve the desired weight of $0.86 \mathrm{~kg}$, the blade was constructed of extruded polystyrene, reinforced with carbon-fiber tubes, and covered with fiberglass. Figures 2.4 and 2.5 show the assembled and cutaway views respectively. The total length of the model is $2.0 \mathrm{~m}$, with $1.5 \mathrm{~m}$ exposed to the flow and $0.5 \mathrm{~m}$ beneath the tunnel. The blade features a NACA $63_{3}-418$ airfoil which tapers from 0.22 -m-chord at the root to $0.11-\mathrm{m}$-chord at the tip. Figure 2.3 shows the model installed at the LSWT.

The center-span section of the blade is fitted with 16 pressure taps distributed around the chord to measure sectional lift. Taps are distributed at 4 additional spanwise locations, each with 3 taps: one at the leading edge and one near the minimum pressure location for both the upper and lower surface. The taps at the center-span will be used to calculate the sectional lift coefficient at this location. 


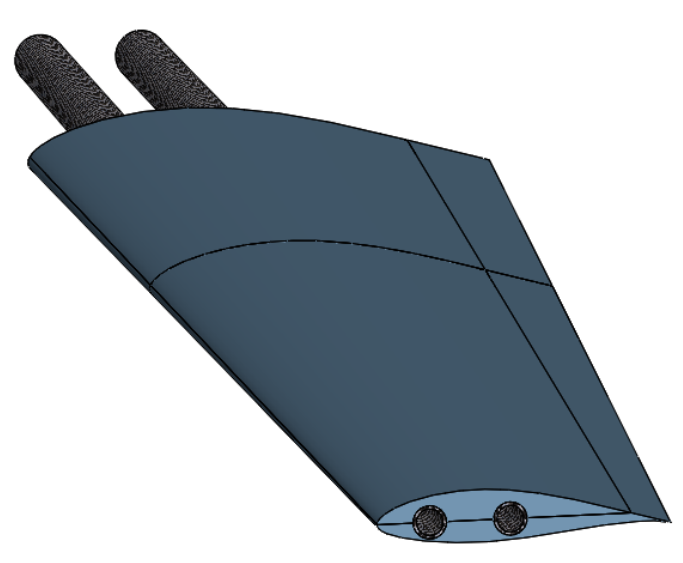

Figure 2.4: Assembled blade.

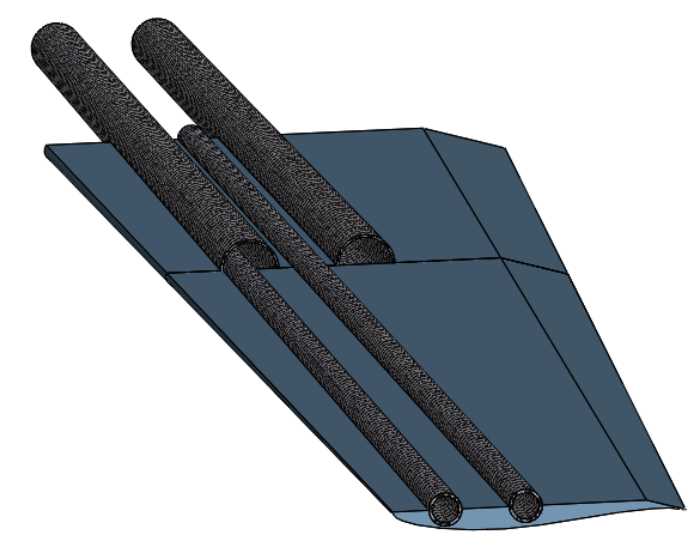

Figure 2.5: Blade model with top pieces removed.

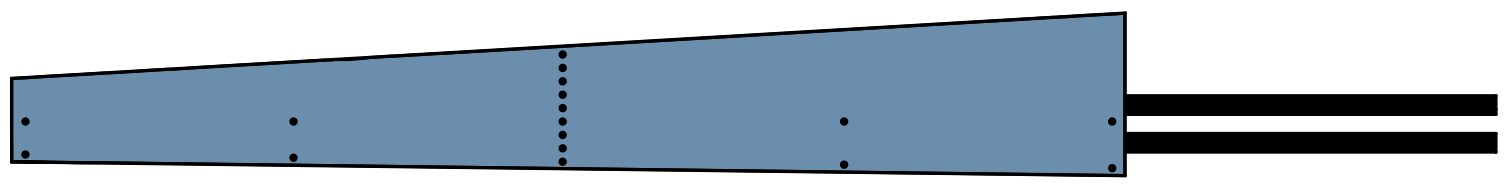

Figure 2.6: Approximate location of pressure taps on blade section.

An MPU-6000 3-axis accelerometer and rate gyroscope is mounted inside the blade, near the center span. This low-cost accelerometer is used in conjunction with strain gauges in the flexible base to measure the total unsteady motion of the blade.

\subsection{Flexible Base}

A cantilever beam, shown in Figure 2.7, provides the spring constants for the two degrees of freedom. The beam consists of a 0.55-m-long, 6.4-mm-diameter steel rod. The rod is cantilevered on one end, and passes through a spherical bearing on the other end. The spherical bearing allows for axial sliding and rotation in all directions. The interface between the beam and blade has bearing blocks, shown in Figure 2.8, mounted fore and aft to restrict edgewise motion. The equivalent mechanical system for the flap mode is shown in Figure 2.9. Two sets of strain gauges are mounted to measure applied moment 


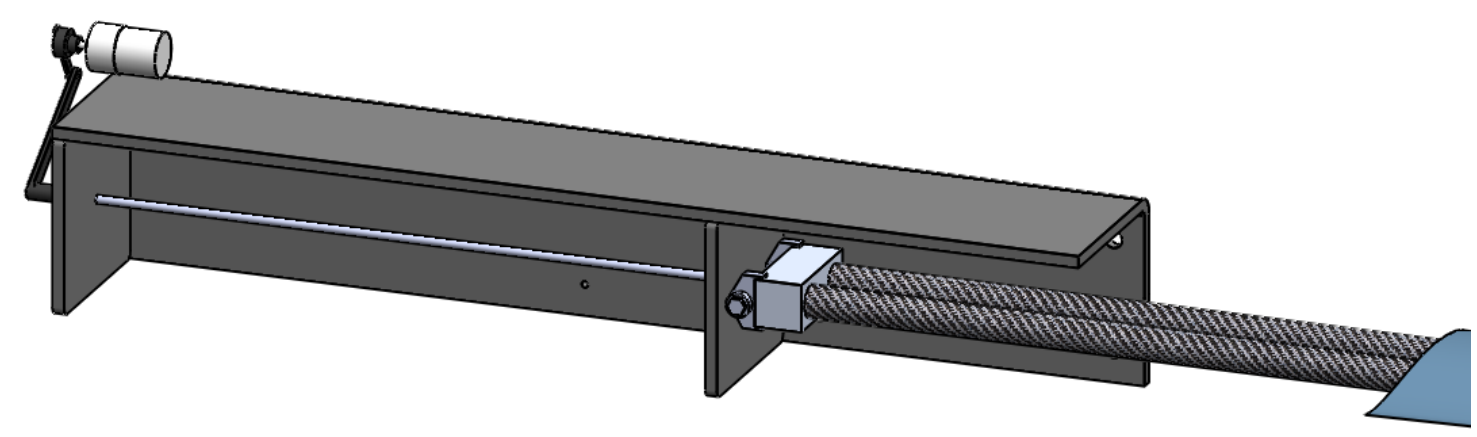

Figure 2.7: Flexible base.

and angular displacement.

\subsection{Pitch Actuator}

The wind tunnel tests were designed to measure the response of the blade to disturbances in the freestream velocity ${ }^{1}$. The freestream velocity is dominated by the by the rotational component, $r \Omega$, which is ideally perpendicular to the wind velocity. A smallamplitude wind-speed perturbation will have negligible effect on the magnitude of the freestream velocity, but will affect angle-of-attack. This can be emulated in a wind-tunnel test in one of two ways: using turning vanes (or some other mechanism) to redirect the freestream, or turning the base of the blade. The two options are slightly different in an aerodynamic sense-the former is modeled by Küssner aerodynamics and the latter is modeled by Wagner aerodynamics. The turning vane approach was rejected in this work for its mechanical complexity, difficulty in ensuring uniform disturbances, and difficulty in accurately quantifying the effect on the flow-field downstream. However, turning vanes more accurately simulate gusts and should be considered for future work.

A pitch actuator was installed at the base of the flexible rod mount. The actuator

\footnotetext{
1 "Freestream velocity" refers to the velocity seen by a section of the blade, i.e. the rotational velocity in addition to the wind velocity. This also refers to the velocity in the wind-tunnel test section.
} 

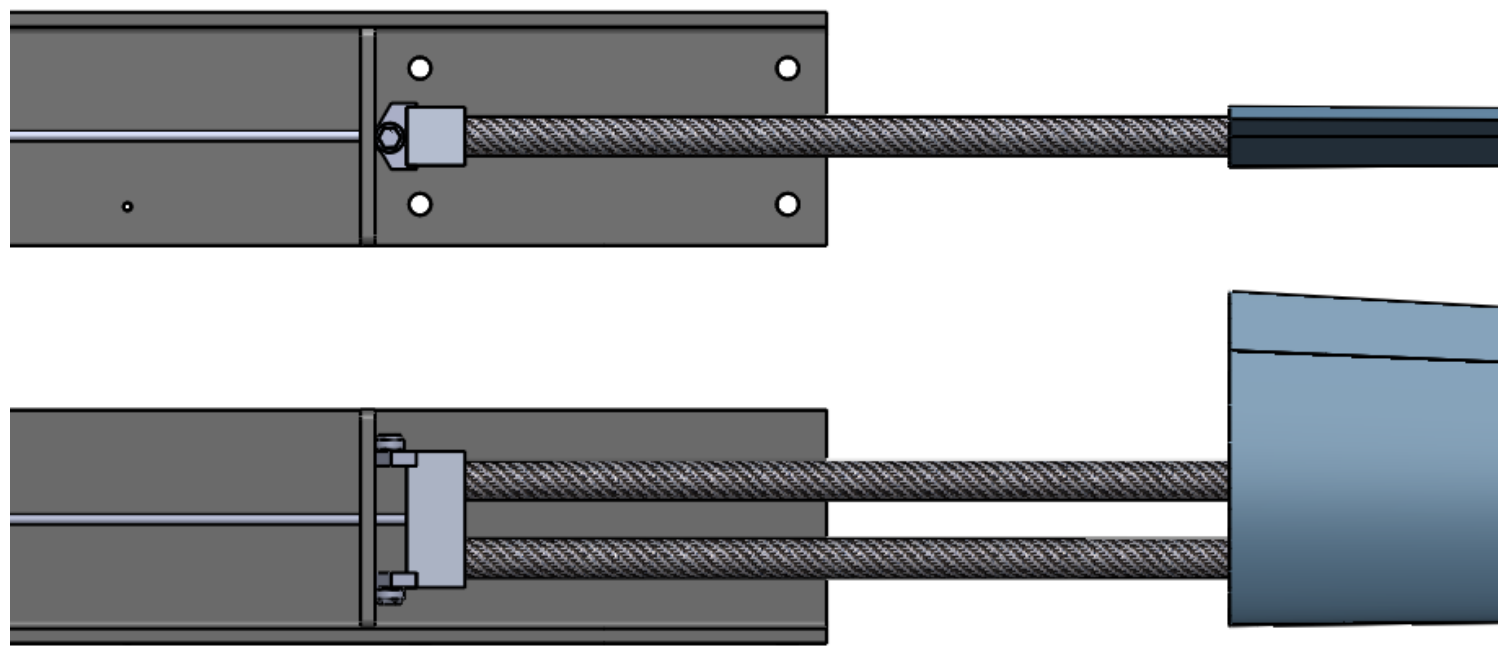

Figure 2.8: Detail views of bearing blocks that restrict edgewise motion.

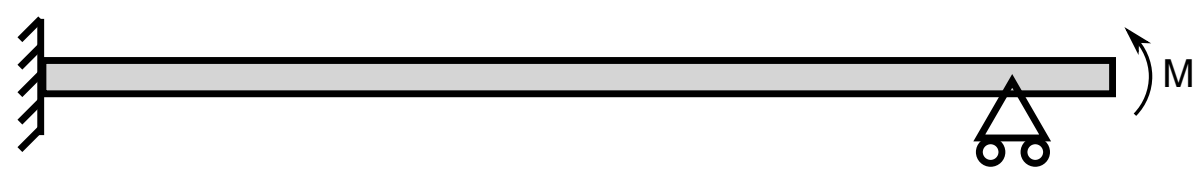

Figure 2.9: Equivalent mechanical system for flapwise bending of rod 


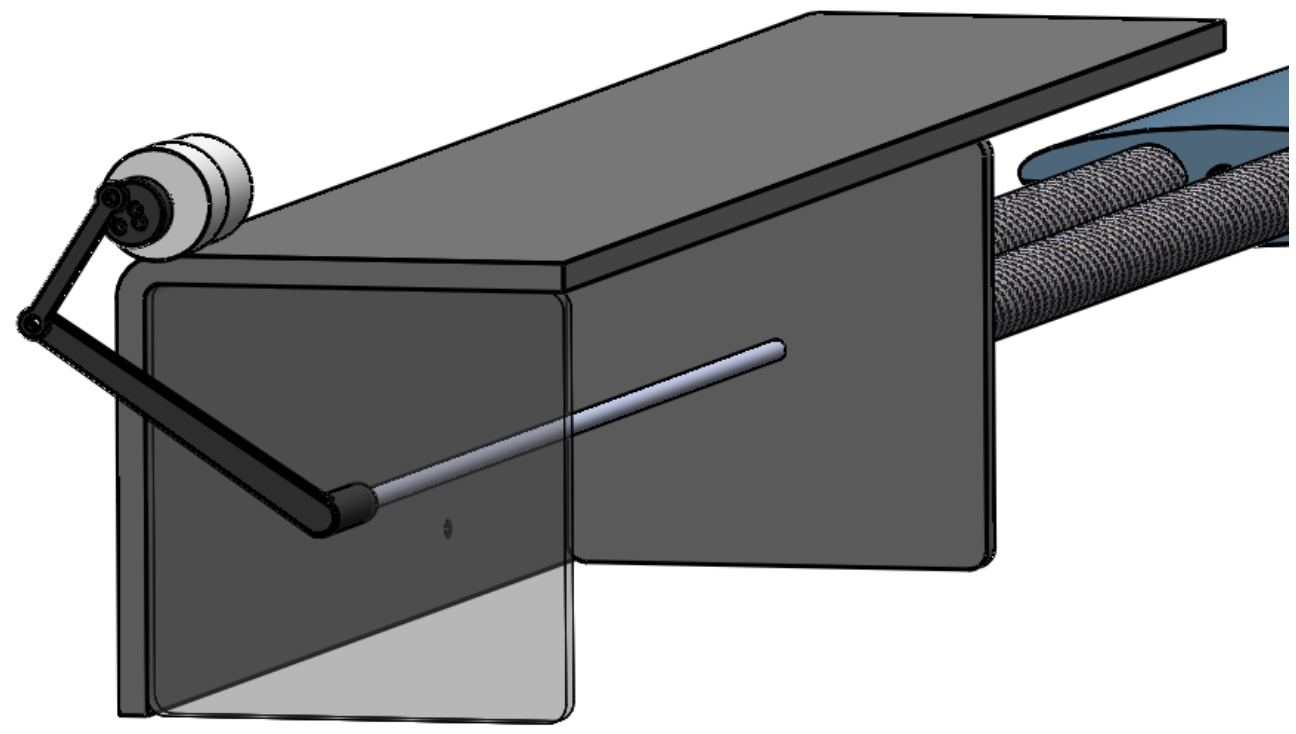

Figure 2.10: Solid model of the pitch actuation mechanism. The crank wheel has 4 different eccentric holes for varying the amplitude of oscillation

consists of a small, geared DC motor driving a crank system shown in Figure 2.10. The motor has a speed range of $1 \mathrm{~Hz}$ to $7 \mathrm{~Hz}$ and drives a crank wheel with different eccentric holes that provide pitch amplitudes of $\pm 1^{\circ}, \pm 2^{\circ}$, and $\pm 4^{\circ}$.

\subsection{Tubing Correction}

Weight and cost requirements for this project preclude the use of pressure transducers mounted inside the model. Instead, pressure taps are connected to tubes running outside the model. Special care must be taken when using long tubes to measure high frequency pressure fluctuations. The pressure measured by a pressure transducer, $p_{t}$, at the end of a tube of length $l$ and inner diameter $d$ is attenuated and phase-shifted from the actual pressure at the end of the tube, $p$. The transfer function for this is given by Bergh and 
Tijdeman [11]:

$$
\begin{aligned}
\frac{p_{t}}{p} & =\left[\cosh (\phi l)+\frac{V_{v}}{\gamma V_{t}} n \phi l \sinh (\phi l)\right]^{-1} \text { where } \\
\phi & =\frac{\omega}{a} \sqrt{\frac{J_{0}(\alpha)}{J_{2}(\alpha)}} \sqrt{\frac{\gamma}{n}} \\
n & =\left[1+\frac{\gamma-1}{\gamma} \frac{J_{2}\left(\alpha \sqrt{P_{r}}\right)}{J_{0}\left(\alpha \sqrt{P_{r}}\right)}\right]^{-1}, \text { and } \\
\alpha & =i \frac{d}{2} \sqrt{\frac{i \omega p_{\infty}}{\mu}}
\end{aligned}
$$

In these equations $\omega$ is the signal frequency, $V_{v}$ is the dead volume of the transducer, $V_{t}$ is the volume inside the tube (calculated from inner diameter and length), $\gamma$ is the specific heat ratio for air, $a$ is the speed of sound in air, $p_{\infty}$ is atmospheric pressure, $P_{r}$ is the Prandtl number, and $J_{m}$ is the Bessel function of the first kind of order $m$. Figure 2.11 shows an example transfer function for tube $1.5 \mathrm{~m}$ long, with a $1 \mathrm{~mm}$ inner diameter, with a transducer dead volume of $0.1 \mathrm{~cm}^{3}$ (approximate values for the tubes used in this experiment). This shows that, up to $100 \mathrm{~Hz}$, the signal is attenuated by no more than a third, which enables pressure reconstruction without excessive noise amplification in the process.

A bench-top calibration rig based on Whitmore et al. [12] was used to validate the transfer function, as well as to estimate the transducer dead volume, which is not readily available. The calibration also provides the tubing length and inner diameter. Comparing these to nominal values can reveal measurement and installation errors such as tubes being pinched or compressed slightly in the model. The setup is outlined in Figure 2.12. An air compressor and regulator are used to pressurize an accumulator tank, which is connected to a solenoid valve. The solenoid leads to a manifold connecting to a reference transducer, a connector needle to insert directly into a pressure tap on the model, and a long piece of tubing vented to the atmosphere (used to prevent resonance in the system). A step-function 

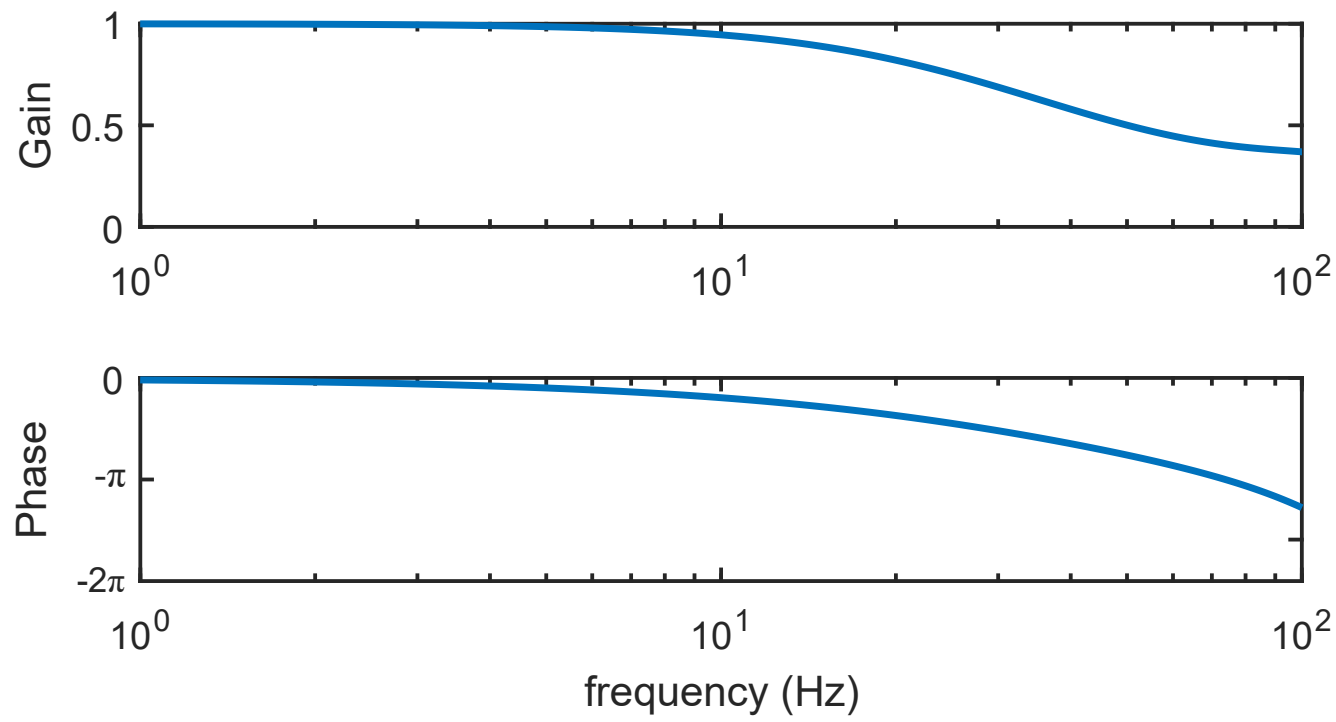

Figure 2.11: Plot of the magnitude of the transfer function for a $1.5 \mathrm{~m}$ tube with an inner diameter of $1.6 \mathrm{~mm}$

pressure wave can be generated by opening the valve. This allows for the determination of the spectral response of all of the odd frequencies up to the Nyquist sampling frequency. The benefit of this configuration is that the compressed air source generates much larger pressure signals compared to acoustic calibration setups, and it is easier to perform in situ.

For each pressure tap, a pressure pulse is generated and a Fourier transform is applied to the measured pressure from the Kulite and the pressure scanner. An example pressure pulse and its Fourier transform are shown in Figures 2.13 and 2.14. The ratios of complex amplitudes of these signals are used as fit data for the transfer function. MATLAB's lsqnonlin is used to perform a nonlinear least-squares estimation of the tubing parameters $l, d$, and $V_{v}$.

\subsection{Aerodynamic Measurements}

The pressure taps on the blade are used to measure the sectional lift coefficient at each of the spanwise locations. First, the pressure measured by each tap (after the above 


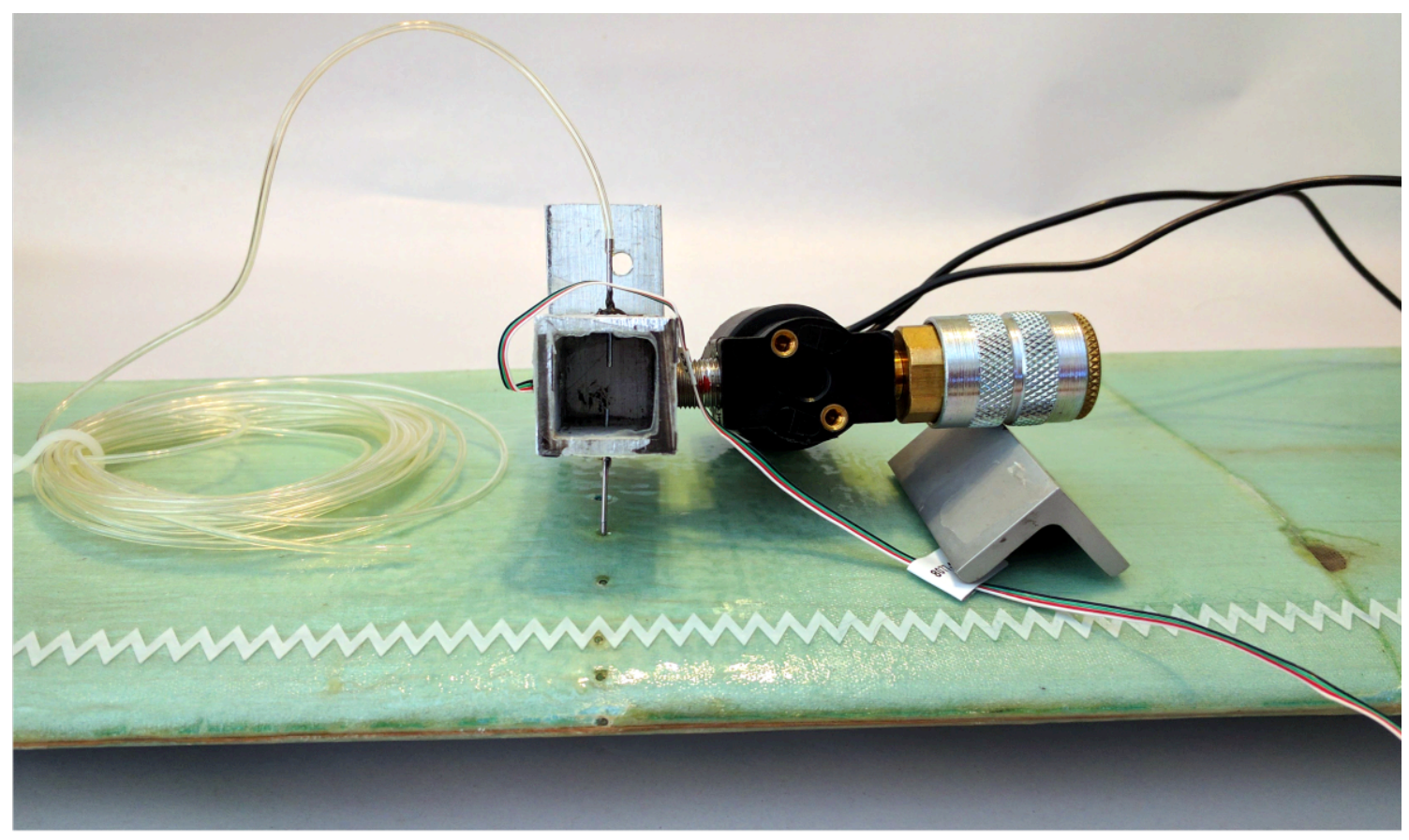

Figure 2.12: Bench-top calibration rig for in situ estimation of tubing parameters.

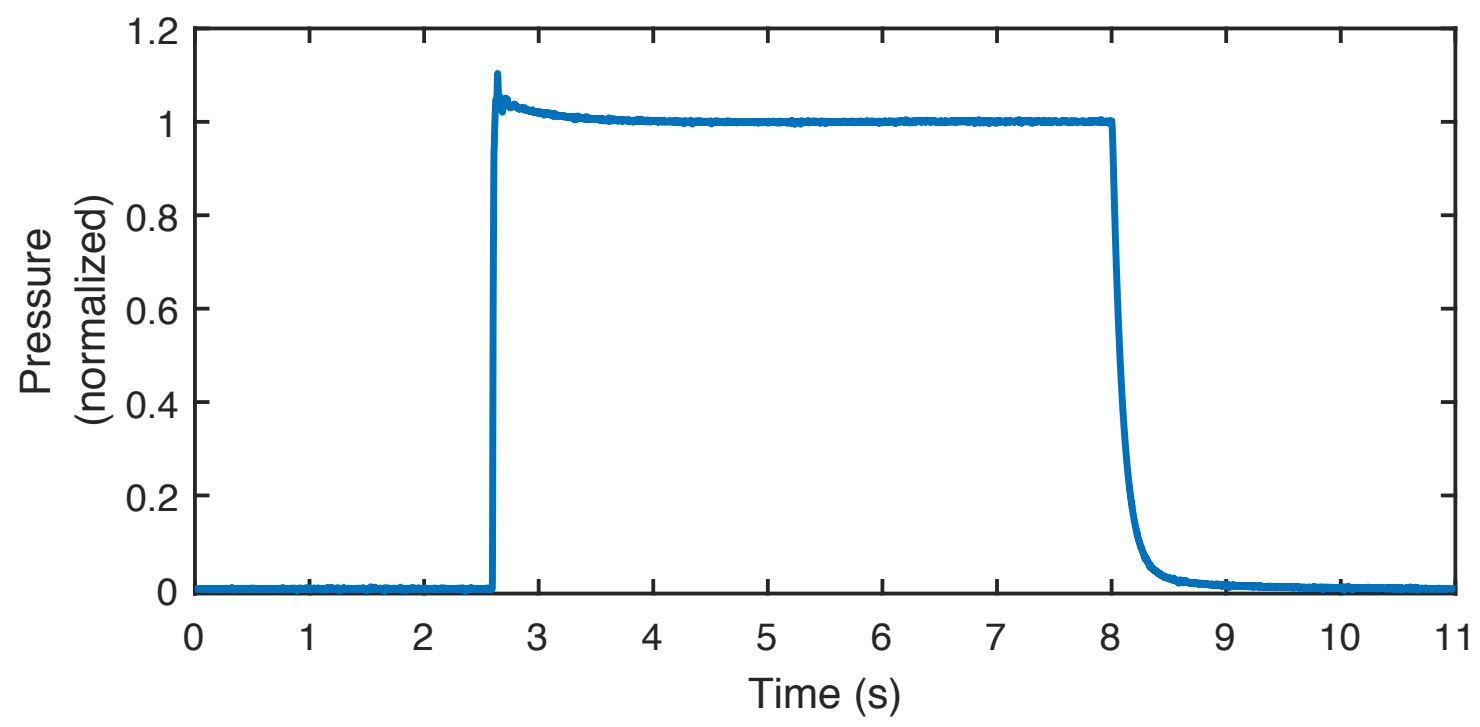

Figure 2.13: Example pressure pulse. There is a slight spike on the front end of the pulse due to resonance. 


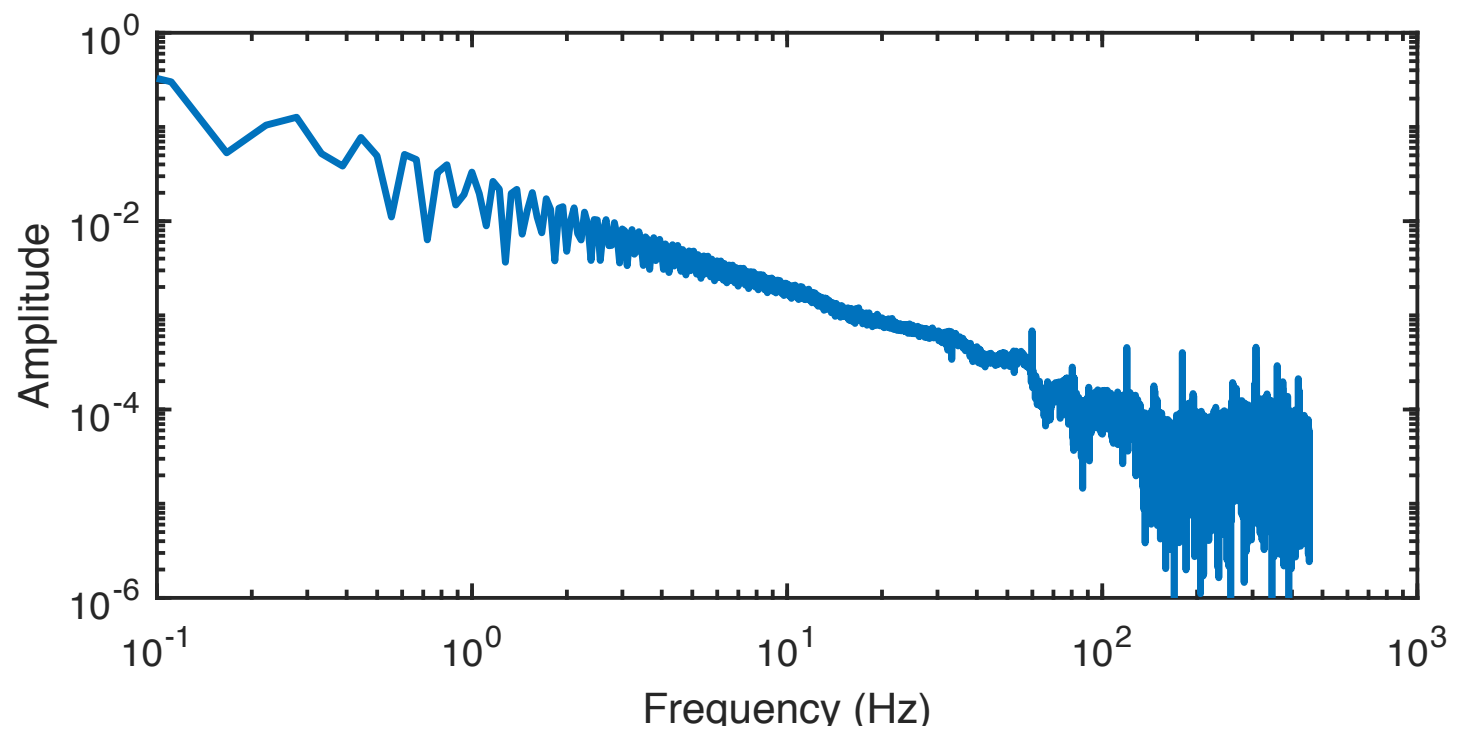

Figure 2.14: Fourier transform of example pressure pulse. The pulse generates reliable signals up to $50 \mathrm{~Hz}$, above which the signal-to-noise ratio becomes too small.

corrections are applied) is converted into a non-dimensional pressure coefficient, $c_{p}$ :

$$
c_{p}=\frac{p-p_{\infty}}{0.5 \rho V^{2}}
$$

The trailing edge of the airfoil is extremely thin, precluding the installation of a pressure tap there. The furthest aft ports are located at $70 \%$ chord on the upper surface, and $85 \%$ chord on the lower surface. In lieu of a trailing-edge tap, the coefficient of pressure for the trailing edge is assumed to be the average of the two aft ports. An example $c_{p}$ distribution is shown in Figure 2.15. From this distribution, assuming small angle of attack, the lift coefficient is found by integrating:

$$
c_{l}=\frac{1}{c} \int_{0}^{c}\left(c_{p, \text { bottom }}-c_{p, \text { top }}\right) d x
$$




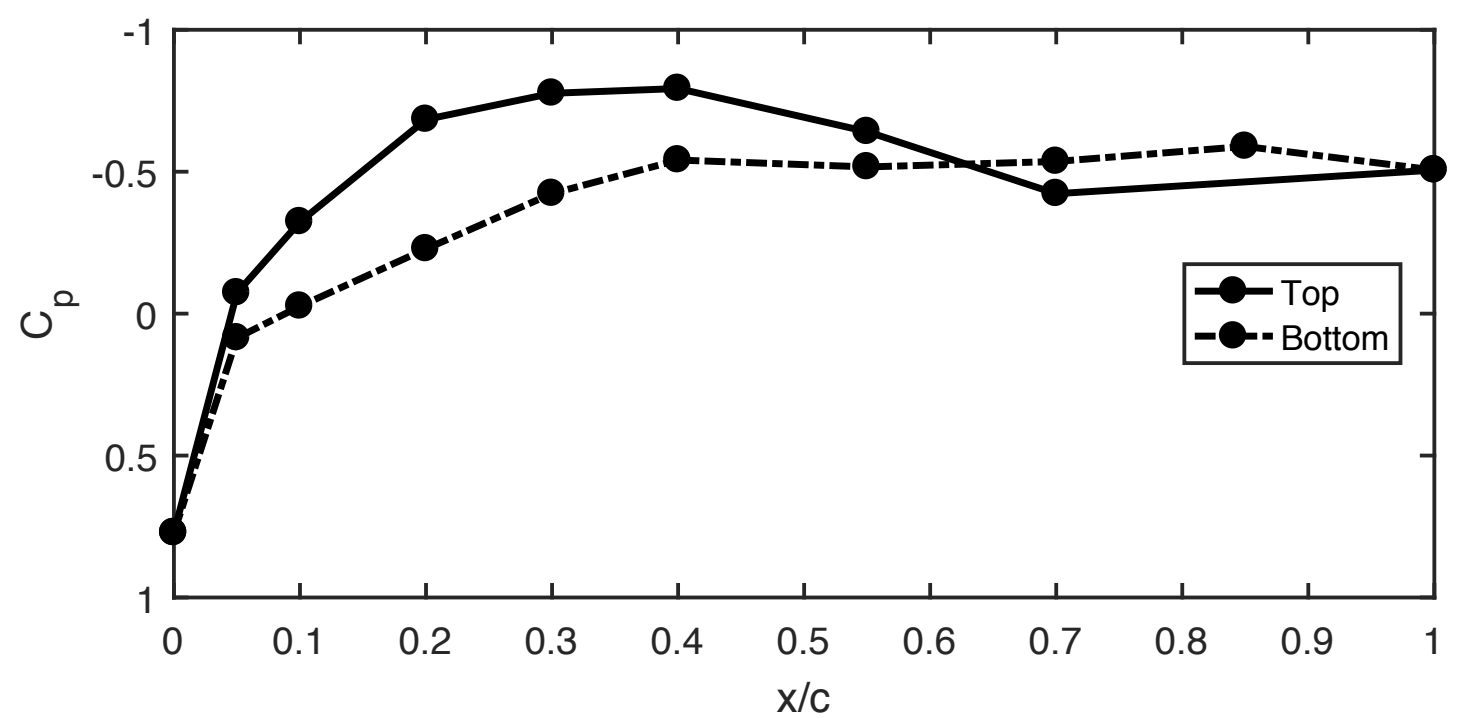

Figure 2.15: An example distribution of pressure over the NACA $63_{3}-418$ at $0.5^{\circ}$ angle of attack.

where $c$ is the chord, $x$ is the distance from the leading edge, $c_{p}$, top and $c_{p}$, bottom are the pressures of the top and bottom surfaces respectively. The taps at the other spanwise locations, with just one port on the upper and lower surface at each location, are far too coarse to properly integrate to find the lift coefficient, however, subtracting the pressure coefficient of the top port from the bottom port should give a rough idea of the lift at each location.

Integrating lift along the blade length yields the out-of-plane flap moment:

$$
M_{\text {aero }}=\int_{r_{0}}^{r_{1}} 0.5 \rho V^{2} r c_{l}(r) d r
$$

where $r$ is the distance of a blade section from the pivot point of the base, $r_{0}$ and $r_{1}$ are the values of $r$ at the root and tip, respectively, of the blade.

The total integrated flap moment can also be measured using the strain gauges. However, unlike static wind-tunnel tests, the model is not in equilibrium, and the forces applied 
at the base are not equal to the aerodynamic load. The method outlined by Babbar [8] is used to measure the aerodynamic loads. The equation of motion for either of the two degrees of freedom is written as

$$
I \ddot{\phi}=M_{\text {aero }}-M_{\text {base }}
$$

where $I$ is the moment of inertia, $\phi$ is the deflection angle, $M_{\text {aero }}$ is the total integrated aerodynamic load, and $M_{b a s e}$ is the load measured by the strain gauges in the flexible base. For static measurements, it's clear that $M_{\text {aero }}=M_{b a s e}$, but for dynamic motion,

$$
M_{\text {aero }}=K \phi+c \dot{\phi}+I \ddot{\phi}
$$

where $K$ is the spring constant at the base and $c$ is the damping coefficient. To measure these loads, it is necessary to estimate the blade motion and all its derivatives. Using accelerometers and rate gyroscopes allows for direct measurement of these derivatives.

\subsection{Data Acquisition}

Figure 2.16 shows the system used to acquire synchronized signals from the accelerometer, pressure taps, and strain gauges. The analog signals from the strain gauges and the pressure scanner were read using an NI-USB 6211 analog to digital converter.

The MPU-6000 is a digital sensor, and cannot be integrated into traditional analogto-digital data acquisition systems. This sensor was chosen for its low cost and high number of features compared to that of traditional accelerometers used at the LSWT. A Microchip ${ }^{\circledR}$ PIC18 microcontroller was used to configure and acquire data from the MPU6000 using an Inter-Integrated Circuit $\left(\mathrm{I}^{2} \mathrm{C}\right)$ serial bus. The MPU-6000 features a digital trigger output for synchronizing data between itself, the pressure scanner, and the strain gauges. When the MPU-6000 has a new sample ready to read from the data buffer, it sends 
a short pulse on the trigger pin. Upon seeing a rising edge on the trigger pin, the PIC18 reads the 12 bytes of accelerometer and gyroscope data and sends it through a serial-toUSB converter to the PC. The MPU-6000 has a nominal sample frequency of $1 \mathrm{kHz}$, but the actual sample frequency for this particular chip was $0.91 \mathrm{kHz}$.

An ESP-32HD was used to measure the pressure from the 28 pressure taps. Five digital inputs control a multiplexer which determines which of transducers is connected to the single analog output of the scanner. Only a single channel can be read at one time, but the multiplexer has a scan rate of $50 \mathrm{kHz}$. A second PIC18 is used to control the pressure scanner. Upon seeing a rising edge on the trigger pin from the MPU-6000, the PIC18 sweeps through the channels and sends 28 trigger pulses, synchronized to the channel changes, to the NI-USB. The NI-USB is configured to take a sample on a rising edge of the trigger output from the second PIC18. The delay between the first and last channel is about $0.6 \mathrm{~ms}$, which is sufficiently short to be considered simultaneous in this test.

Data is collected in batches of 10,000 points (11 seconds). The MPU-6000 is placed

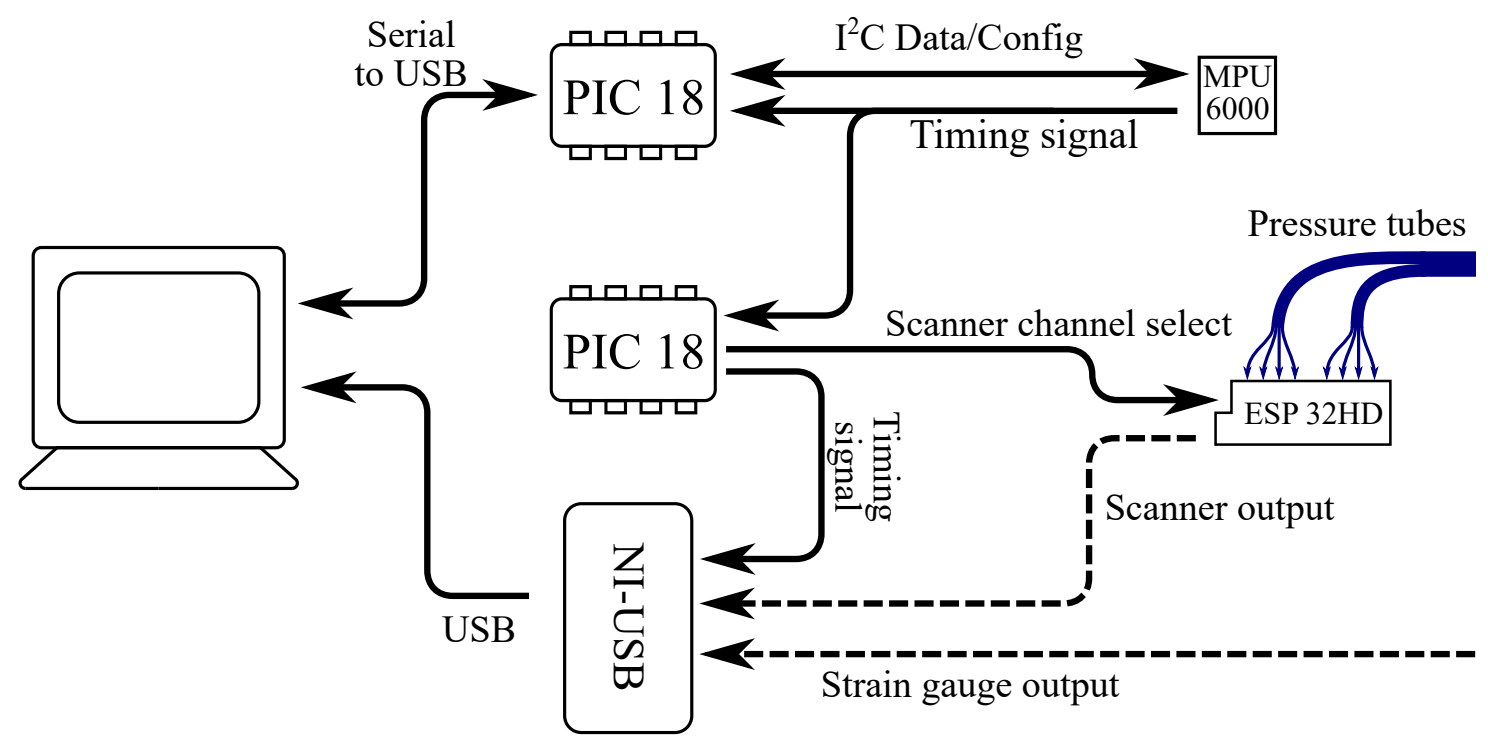

Figure 2.16: Diagram of electronics used for data acquisition. 
in sleep mode between batches. A command is sent from the PC to the first PIC18 to start data collection. Once this is received, the PIC18 places the MPU-6000 in operate mode and waits for each trigger pulse. At the same time, a LabVIEW ${ }^{\circledR}$ script is started to collect data from the scanner and strain gauges. For each sample taken from the MPU-6000, 28 samples are read by the NI-USB. For the scanner input, each of these samples represents a different channel; for the two strain gauge inputs, each set of 28 samples is averaged to create one point per accelerometer reading. 


\section{PREDICTIVE MODELS}

One objective of this work is to develop a time-domain aeroelastic model for a finitespan cantilevered blade. Frequency domain models are popular for flutter prediction, as well as simulation of naturally periodic systems, such as flapping wing flight. However, turbine designers are not primarily concerned with flutter, and not all disturbances experienced by the blades are periodic. Transient gust responses are of great importance. Additionally, although wind turbine blades have a high aspect ratio, the most important aerodynamic region is near the tip of the blade, where 3D end effects cannot be neglected. This section presents the development of an aeroelastic based on Wagner's aerodynamics and lifting line theory.

\subsection{Unsteady 2D Thin Airfoils}

Before a 3D model can be constructed, the underlying 2D aerodynamics of the airfoil sections must be modeled. The $2 \mathrm{D}$ unsteady model has the following assumptions and requirements: inviscid, incompressible, thin airfoil, calculate circulatory lift, and timedomain linear ordinary differential equation (ODE). The first two requirements are acceptable for high Reynolds number and Mach numbers below 0.3 , both of which are satisfied on a full-scale turbine. For structural reasons, the airfoil sections near the root are quite thick, but this work focuses on the outboard end of the blade, so the thin airfoil assumption is acceptable. The fourth item states that the total circulation of the airfoil must be modeled, not just the total lift force. In steady flow, these are linearly related, but unsteady flow introduces extra terms that are independent of circulation. The circulatory component of lift will be necessary when extending to lifting-line theory as it determines the influence of neighboring airfoil sections. The final requirement allows the aerodynamics to be written as a state-space system, which will be useful when extending to 3D aerodynamics. 


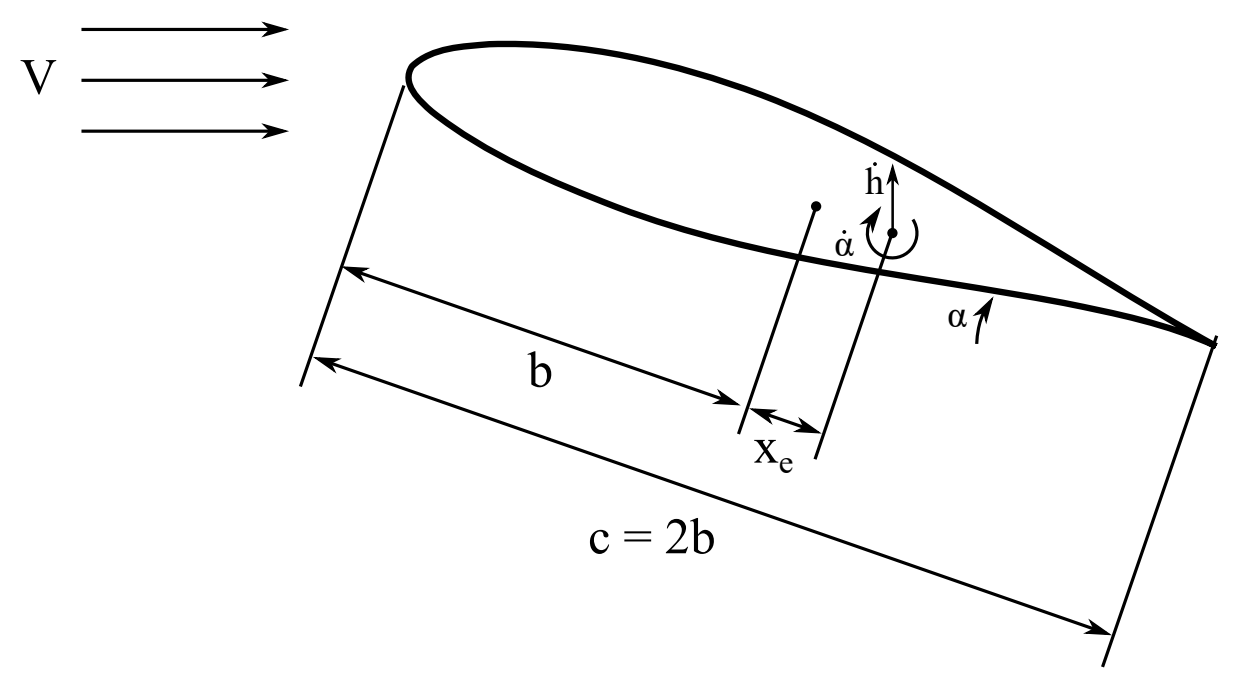

Figure 3.1: Standard nomenclature for 2D unsteady airfoil theory.

Figure 3.1 shows the standard nomenclature used for a thin airfoil undergoing pitching and plunging motion. $b$ is the semi-chord length, $\alpha$ is the angle of attack, and $h$ is the plunge motion of the airfoil. The plunge motion is measured at the point located at $x_{e}$, also known as the elastic axis.

Quasi-steady aerodynamics work well when the terms $b \dot{\alpha}$ and $\dot{h}$ are small compared to the freestream velocity $V$. It is assumed that the airfoil sees a steady angle of $\alpha+\dot{h} / V$ and the zero-penetration condition is modified for a constant $\dot{\alpha}$. This results in the lift coefficient

$$
c_{l_{\text {q.s. }}}=2 \pi\left[\alpha+\frac{\dot{h}}{V}+\left(\frac{c}{4}-x_{e}\right) \frac{\dot{\alpha}}{V}\right]
$$

The terms in the brackets above are commonly referred to as the quasi-steady angle-ofattack.

Theodorsen developed a frequency-domain unsteady aerodynamics model that is frequently used in flutter stability analysis [13]. The pitch, $\alpha$, and plunge, $h$, are represented as sinusoids of a constant frequency with magnitudes and phase angles defined by the 
complex variables $\bar{\alpha}$ and $\bar{h}$.

$$
\alpha=\bar{\alpha} e^{i k \frac{V}{b} t}
$$

where the imaginary part of $\alpha$ is ignored. The reduced frequency of the oscillation is denoted by $k=\omega b / V$. Theodorsen's equation for lift is

$$
c_{l_{\text {theo }}}=\underbrace{\frac{\pi b}{V^{2}}\left(\ddot{h}-x_{e} \ddot{\alpha}+V \dot{\alpha}\right)}_{\text {added-mass terms }}+\underbrace{C(k) \bar{C}_{l_{q . s .}} e^{i k \frac{V}{b} t}}_{\text {circulatory term }}
$$

$C(k)$ is known as the Theodorsen function; it is a complex function which defines an attenuation and phase shift of the quasi-steady lift given a reduced frequency $k$. The ultimate goal is to arrive at a time-domain model. As seen in equation 3.3, the inertial aerodynamic forces, or "added-mass" terms, are already in the form of a time-domain, linear ODE. A time-domain solution must be found for the circulatory lift only. For this, Wagner's aerodynamics are used.

Wagner developed a model for the lift response of an impulsively started thin airfoil [14]. The solution is based on thin airfoil theory in the presence of shed vorticity, which starts at the trailing edge, and moves downstream at the freestream velocity. For a thin airfoil impulsively accelerated from rest to a constant velocity $V$, the lift is given by

$$
c_{l}=2 \pi \alpha \Phi\left(\frac{V t}{b}\right)
$$

where $\Phi(\tau)$ is known as Wagner's function, which can be calculated by an integral equation

$$
\Phi(\tau)=\frac{2}{\pi} \int_{0}^{\infty} \frac{F(k) \sin (k \tau)}{k} d k
$$

where $F(k)$ is the real part of the Theodorsen function. This integral can be evaluated 
numerically, but an exponential approximation is commonly used [15].

$$
\Phi(\tau)=1-0.165 e^{-0.0455 \tau}-0.335 e^{-0.3 \tau}
$$

This approximation is accurate within $1 \%$ for all values of $\tau$.

The exponential approximation is used to transform the step response of the airfoil into a system of ordinary differential equations. First, $\Phi$ is rewritten as

$$
\Phi=\Psi_{0}+\Psi_{1}\left(1-e^{-\epsilon_{1} \frac{V t}{b}}\right)+\Psi_{2}\left(1-e^{-\epsilon_{2} \frac{V t}{b}}\right)
$$

where $\Psi_{0}=0.5, \Psi_{1}=0.165, \Psi_{2}=0.335, \epsilon_{1}=0.0455$, and $\epsilon_{2}=0.3$. The exponential parts of the above function are recognized as the step response of a first-order linear differential equation. Using this, the instantaneous section lift is given by a system of three linear ODEs.

$$
\begin{aligned}
& c_{l}=2 \pi\left(z_{1}+z_{2}+\Psi_{0} \alpha\right) \\
& \dot{z}_{1}=-\epsilon_{1} \frac{V}{b}\left(z_{1}-\Psi_{1} \alpha\right) \\
& \dot{z}_{2}=-\epsilon_{2} \frac{V}{b}\left(z_{2}-\Psi_{2} \alpha\right)
\end{aligned}
$$

The initial conditions are $z_{1}(0)=0$ and $z_{2}(0)=0$.

Though not used in this work, Küssner developed a similar step response function for an airfoil traveling through a sharp gust. An approximation to Küssner's function exists in the same form as equation 3.7, but with $\Psi_{0}=0, \Psi_{1}=0.5, \Psi_{2}=0.5, \epsilon_{1}=0.13$, and $\epsilon_{2}=1.0$ [15]. In future work involving gust generators, equation 3.8 could be reformulated to simulate the gust response. 


\subsection{Unsteady Lifting Line Theory}

The 3D extension of Wagner's aerodynamics, based on the methods of Boutet and Dimitriadis [16] are presented here, with some improvements to computational efficiency.

A Fourier series is used to represent the spanwise distribution of the time-varying circulation on the blade.

$$
\Gamma(t, \theta)=\frac{c_{0} U}{2} \sum_{n=1}^{j} a_{n}(t) \sin (n \theta)
$$

Where $c_{0}$ is the chord at the base of the blade, $j$ is the number of terms in the truncated series. The linear spanwise coordinate, $y$ is tranformed into an angular coordinate $\theta$ such that $y=s(1-\cos \theta)$, where $\theta$ varies from 0 to $\pi$ from left to right. The blade is cantilevered, but the effect of the tunnel floor is modeled as a mirror image to create a symmetric wing with a span of $2 s$. Because the simulated wing is symmetric, only the odd values of $n$ are needed.

For a 2D airfoil section with a lumped vortex of $\Gamma$ at the quarter-chord, the sectional lift coefficient is given by

$$
c_{l}=\frac{2 \Gamma}{U c}+\frac{2 \dot{\Gamma}}{U^{2}}=\sum_{n=1}^{j}\left(\frac{c_{0}}{c} a_{n}+\frac{c_{0}}{U} \dot{a}_{n}\right) \sin (n \theta)
$$

The downwash induced by a differential vortex element located at $y_{0}$ onto an element located at $y$ is

$$
\begin{aligned}
d w & =\frac{\left.d \Gamma\right|_{y_{0}}}{4 \pi\left(y-y_{0}\right)} \\
& =\frac{c_{0} U}{8 \pi} \sum_{n=1}^{j} n a_{n} \frac{\cos \left(n \theta_{0}\right)}{s \cos \left(\theta_{0}\right)-s \cos (\theta)} d \theta_{0}
\end{aligned}
$$


which is integrated to find the downwash distribution

$$
w=\frac{c_{0} U}{8 \pi s} \sum_{n=1}^{j} n a_{n} \int_{0}^{\pi} \frac{\cos \left(n \theta_{0}\right)}{\cos \left(\theta_{0}\right)-\cos (\theta)} d \theta_{0}
$$

The integral in the above equation is known as Glauert's integral, and has a known solution

$$
\begin{gathered}
\int_{0}^{\pi} \frac{\cos \left(n \theta_{0}\right)}{\cos \left(\theta_{0}\right)-\cos (\theta)} d \theta_{0}=\frac{\pi \sin (n \theta)}{\sin (\theta)} \\
w(t, \theta)=\frac{c_{0} U}{8 s} \sum_{n=1}^{j} \frac{n a_{n}(t) \sin (n \theta)}{\sin (\theta)}
\end{gathered}
$$

Given a rigid blade capable of angular motion in the flap and twist directions, the total induced angle of attack at a location $\theta$ is given by

$$
\alpha^{\mathrm{tot}}(t, \theta)=\phi_{t}(t)+\frac{\frac{1}{2} b(\theta)-x_{e}(\theta)}{U} \dot{\phi}_{t}(t)-\frac{R+|s \cos (\theta)|}{U} \dot{\phi}_{f}(t)-\frac{w(t, \theta)}{U}
$$

where $\phi_{f}$ and $\phi_{t}$ are the flap and twist angles of the blade, respectively. $R$ is the distance of the base of the blade from the axis of rotation for the flap degree of freedom.

The circulatory lift of the Fourier series is equated to that found in the previous section for Wagner's aerodynamics.

$$
c_{l}(t, \theta)=c_{0} \sum_{n=1}^{j}\left(\frac{a_{n}}{c}+\frac{\dot{a}_{n}}{U}\right) \sin (n \theta)=z_{1}(t, \theta)+z_{2}(t, \theta)+0.5 C_{l_{\alpha}} \alpha^{\mathrm{tot}}(t, \theta)
$$

This equation can be evaluated at $j$ locations of the span to generate ordinary differential 
matrix equations (represented in Einstein summation notation)

$$
\begin{aligned}
M_{i n} \dot{a}_{n} & =K_{i n} a_{n}+2 \pi\left(z_{1_{i}}+z_{2_{i}}+\Psi_{0} G_{i k} u_{k}\right) \\
\dot{z_{1_{i}}} & =-\epsilon_{1} \frac{U}{b_{i}}\left(\Psi_{1} W_{i n}-z_{1_{i}}+\Psi_{1} G_{i k} u_{k}\right) \\
\dot{z_{2_{i}}} & =-\epsilon_{2} \frac{U}{b_{i}}\left(\Psi_{2} W_{i n}-z_{2_{i}}+\Psi_{2} G_{i k} u_{k}\right)
\end{aligned}
$$

where

$$
\begin{gathered}
M_{i n}=\frac{c_{0}}{U} \sin \left(n \theta_{i}\right) \\
K_{i n}=-\frac{c 0}{c_{i}} \sin \left(n \theta_{i}\right)-2 \pi \Psi_{0} W_{i n} \\
W_{i n}=c_{0} \frac{n}{8 s} \frac{\sin \left(n \theta_{i}\right)}{\sin \left(\theta_{i}\right)} \\
G_{i k}=\left[\begin{array}{ll}
1, & \left.\frac{b_{i}-2 x_{e_{i}}}{2 U}, \quad-\frac{R+s \cos \left(\theta_{i}\right)}{U}\right]_{k} \\
u_{k}=\left[\begin{array}{l}
\phi_{t} \\
\dot{\phi}_{t} \\
\dot{\phi}_{f}
\end{array}\right]_{k}
\end{array}\right.
\end{gathered}
$$

The circulatory lift is now expressed as a first-order linear ordinary differential equation, and can be numerically simulated given known inputs $\phi_{f}(t)$ and $\phi_{t}(t)$. Once $a_{n}$ and $\dot{a}_{n}$ are calculated, the added-mass terms from Theodorsen's must be added to the circulatory lift to get total lift. That is, the lift coefficient is given by

$$
c_{l}=\sum_{n}\left[\left(\frac{c_{0}}{c} a_{n}+\frac{c_{0}}{U} \dot{a}_{n}\right) \sin (n \theta)\right]+\pi \frac{b}{U^{2}}\left(-r \ddot{\phi}_{f}-x_{e} \ddot{\phi}_{t}+U \dot{\phi}_{t}\right)
$$

Figure 3.2 shows how the lift coefficient in the above equation evolves for a step change in twist angle. The sectional lift distribution over the span at various times is plotted as well as the total integrated lift as a function of time. The geometry of the blade described 
in section 2.3 is used in this simulation. The elastic axis location, $x_{e}$, varies linearly from $-0.04 \mathrm{~m}$ at the root, to $-0.005 \mathrm{~m}$ at the tip.

\subsection{Simulation of Motion}

The previous section assumes the flap and twist motions are known. This section will take one more step and model the flap response, as well as the loads, given a known input of the twisting motion.

First, equation 3.17 is rewritten in state-space form, with the integrated flap moment, $M_{\text {flap }}$ as the output.

$$
\begin{aligned}
& \dot{x}=A x+B u \\
& y=C x+D u
\end{aligned}
$$

where

$$
\begin{aligned}
& x=\left[\begin{array}{lll}
a_{n} & z_{1_{i}} & z_{2_{i}}
\end{array}\right]^{\top} \\
& u=\left[\begin{array}{lllll}
\phi_{t} & \dot{\phi}_{t} & \ddot{\phi}_{t} & \dot{\phi}_{f} & \ddot{\phi}_{f}
\end{array}\right]^{\top} \\
& y=M_{\text {flap }}
\end{aligned}
$$

The $A$ and $B$ matrices can be easily found using equation 3.17. $M_{\text {flap }}$ is found by integrating equation 3.23 multiplied by the distance from the axis of rotation.

$$
\begin{array}{r}
M_{\text {flap }}=\int_{0}^{s} \rho U^{2} b(R+y)\left\{\sum_{n}\left[\left(\frac{c_{0}}{c} a_{n}+\frac{c_{0}}{U} \dot{a}_{n}\right) \sin (n \theta)\right]+\right. \\
\left.\pi \frac{b}{U^{2}}\left(-r \ddot{\phi}_{f}-x_{e} \ddot{\phi}_{t}+U \dot{\phi}_{t}\right)\right\} d y
\end{array}
$$

where $R$ is the distance of the root of the blade from the axis of rotation. From this $C$ and 

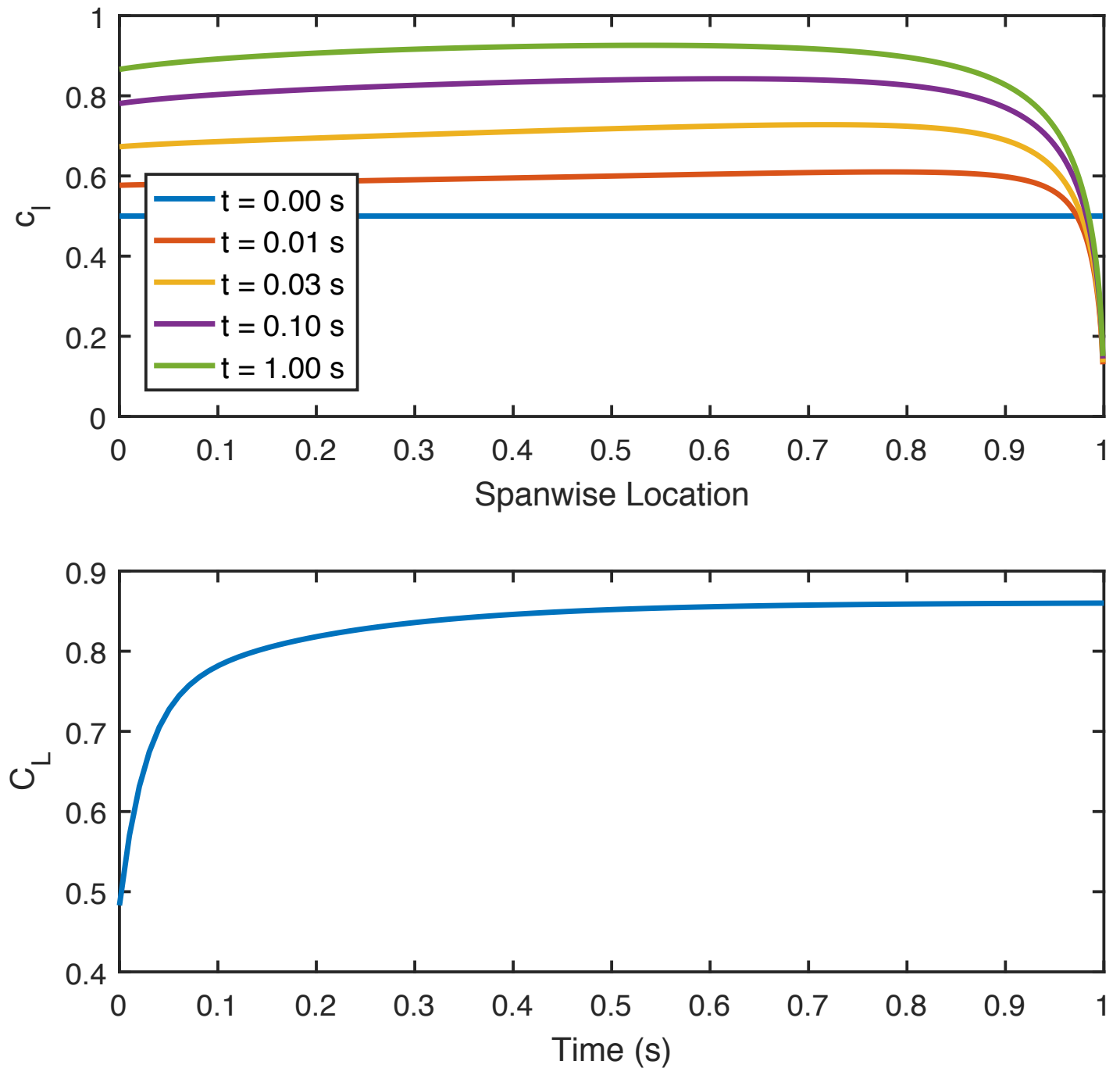

Figure 3.2: Top: lift coefficient distribution along the span at various times for a step change in twist angle. Bottom: integrated lift coefficient over whole blade versus time. 
$D$, which are single-row matrices, can be written

$$
\begin{aligned}
C_{n} & =\rho U^{2} b_{0}\left(E_{n}+F_{k} K_{k n}\right) \\
D_{n} & =\rho U^{2} b_{0}\left(F_{k} B_{k n}+H_{n}\right) \\
E_{n} & =\int_{0}^{s}(R+y) \sin (n \theta) d y \\
F_{n} & =\frac{2}{U} \int_{0}^{s} b(R+y) \sin (n \theta) d y \\
H & =\frac{\pi}{b_{0} U} \int_{0}^{s} b^{2}(R+y)\left[\begin{array}{lllll}
0 & 1 & -\frac{x_{e}}{U} & 0 & -\frac{R+y}{U}
\end{array}\right] d y
\end{aligned}
$$

Next, $\phi_{f}$ and $\dot{\phi}_{f}$ are added to the state vector and a new state-space system is derived.

$$
\begin{gathered}
\dot{x}^{*}=A^{*} x^{*}+B^{*} u^{*} \\
y=C^{*} x^{*}+D^{*} u^{*} \\
x^{*}=\left[\begin{array}{lllll}
a_{n} & z_{1_{i}} & z_{2_{i}} & \phi_{f} & \dot{\phi}_{f}
\end{array}\right]^{\top} \\
u^{*}=\left[\begin{array}{lll}
\phi_{t} & \dot{\phi}_{t} & \ddot{\phi}_{t}
\end{array}\right]^{\top} \\
A^{*}=\left[\begin{array}{ccc}
A & 0 & B_{n 4} \\
0 & 0 & 1 \\
\frac{C}{I_{\text {flap }}-D_{5}} & -\frac{K_{\text {flap }}}{I_{\text {flap }}-D_{5}} & \frac{D_{4}-2 \xi_{\text {flap }} \sqrt{K_{\text {flap }} I_{\text {flap }}}}{I_{\text {flap }}-D_{5}}
\end{array}\right] \\
B^{*}=\left[\begin{array}{ccc}
B_{n 1} & B_{n 2} & B_{n 3} \\
0 & 0 & 0 \\
\frac{D_{1}}{I_{\text {flap }}-D_{5}} & \frac{D_{2}}{I_{\text {flap }}-D_{5}} & \frac{D_{3}}{I_{\text {flap }}-D_{5}}
\end{array}\right]
\end{gathered}
$$

Figure 3.3 shows the flap response for a step change of $1^{\circ}$ in twist. Different numbers of even modes used in the lifting line Fourier series, $N=(j+1) / 2$, are compared. This plot shows that 10 modes are sufficient for subsequent simulations. 


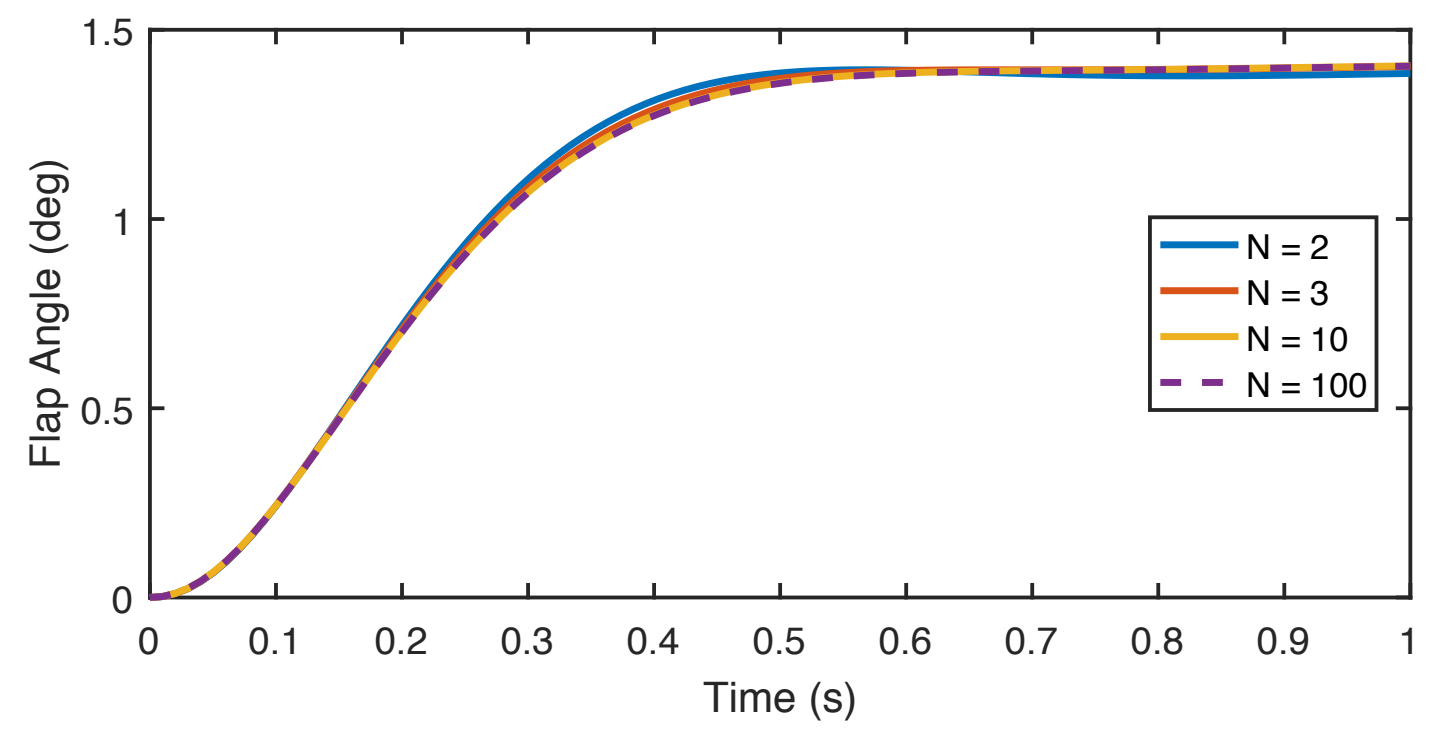

Figure 3.3: Flap motion for a step change in twist of $1^{\circ}$ for various numbers of even modes used in the simulation, $N$.

This same process could be performed for the twist degree of freedom, but the design of the elastic base precludes measurement of the integrated twisting moment. Figure 3.4 highlights the problem. With large flap deflections, the edgewise moment (caused by drag on the blade) begins to "bleed" into the twist degree of freedom. The drag for these experiments is not measured, so this effect cannot not be factored out from the data. Future research will need to rethink this aspect of the setup. 


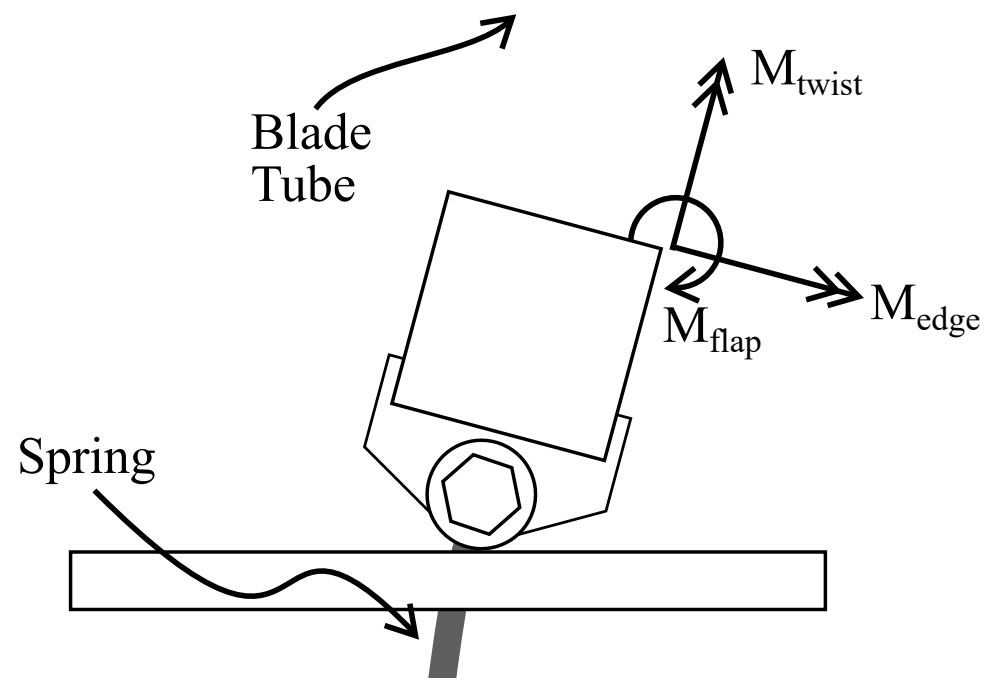

Figure 3.4: Diagram of the mounting bracket, viewed from downstream and looking upstream. This illustrates why integrated twisting moment cannot be measured in these experiments. The unmeasured $M_{e d g e}$ component twists the torsional spring at large angles of flap deflection. 


\section{RESULTS}

That chapter presents the results of the wind tunnel tests on the aeroelastic blade presented in chapter 2 compared to output from the aeroelastic simulation model. This chapter consists of three sections. First, system identification tests are performed to estimate important physical properties of the test article. Next, static pitch sweeps are performed to verify the installed angle of the blade. The main section of this chapter presents the measured and predicted loads and flap motion in response to disturbance of varying amplitudes and reduced frequencies provided by the pitch actuator. The model performance is also compared to the performance of two other predictive models: quasi-steady lifting line, and strip theory with Theodorsen aerodynamics.

\subsection{System Identification}

Before performing wind-on experiments, various system identification tests were performed to estimate properties of the wind-tunnel model mechanical system. First, spring constants for flap and twist motion were measured by laying the blade assembly on its side and placing known weights at various radial locations. A digital inclinometer was used to measure the deflection angle. Strain gauge voltages were measured as well, and used to calibrate them for displacement and torque. Figures 4.1-4.4 show the fit data for the various spring constants and strain gauge gains. Using linear regression the spring constants are found: $K_{\text {flap }}=74 \pm 2 \mathrm{~N} \cdot \mathrm{m}$ and $K_{\text {twist }}=7.6 \pm 0.1 \mathrm{~N} \cdot \mathrm{m}$.

To measure the moment of inertia of each degree of freedom, a series of impulse response tests were conducted with the blade installed in the test section. These responses were generated by installing the blade assembly in the wind tunnel test section and, with the wind off, lightly striking the model by hand to excite motion for the desired degree of freedom. These tests also allowed for measurement of damping coeffi- 


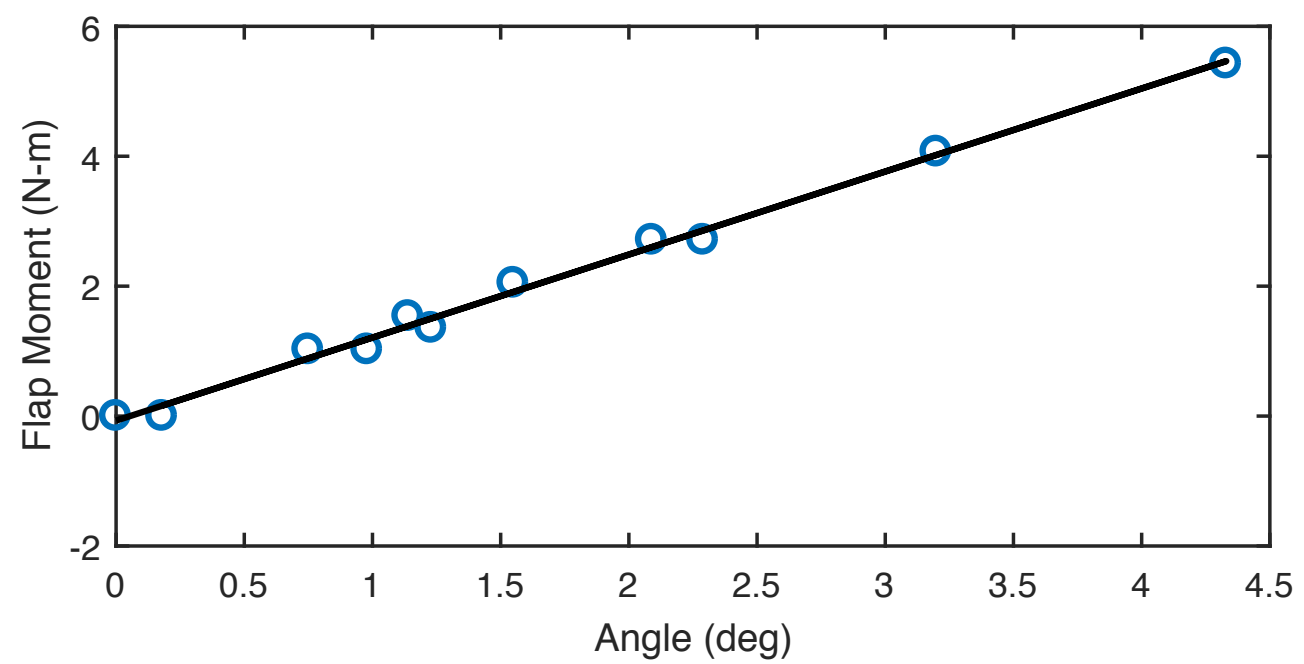

Figure 4.1: Measurement of spring constant for flap motion. Note the slight hysteresis caused by plastic deformation.

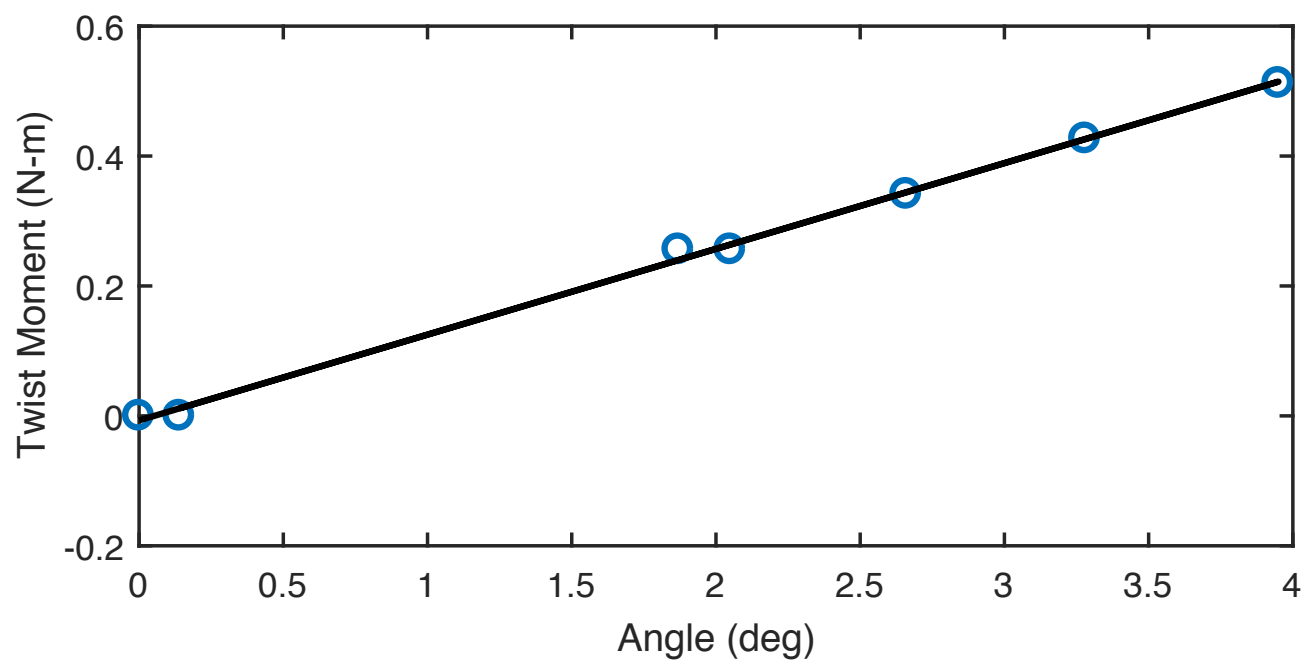

Figure 4.2: Measurement of spring constant for twist motion. Note the slight hysteresis caused by plastic deformation. 


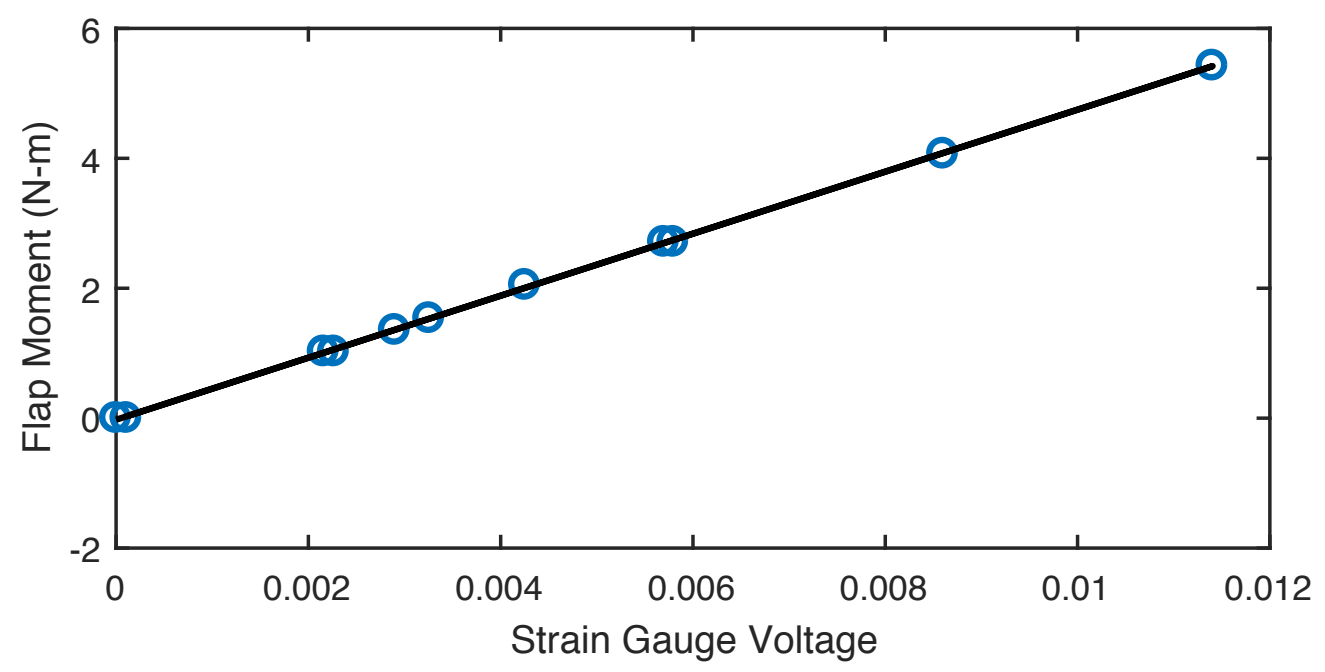

Figure 4.3: Measurement of strain gauge gain for flap moment.

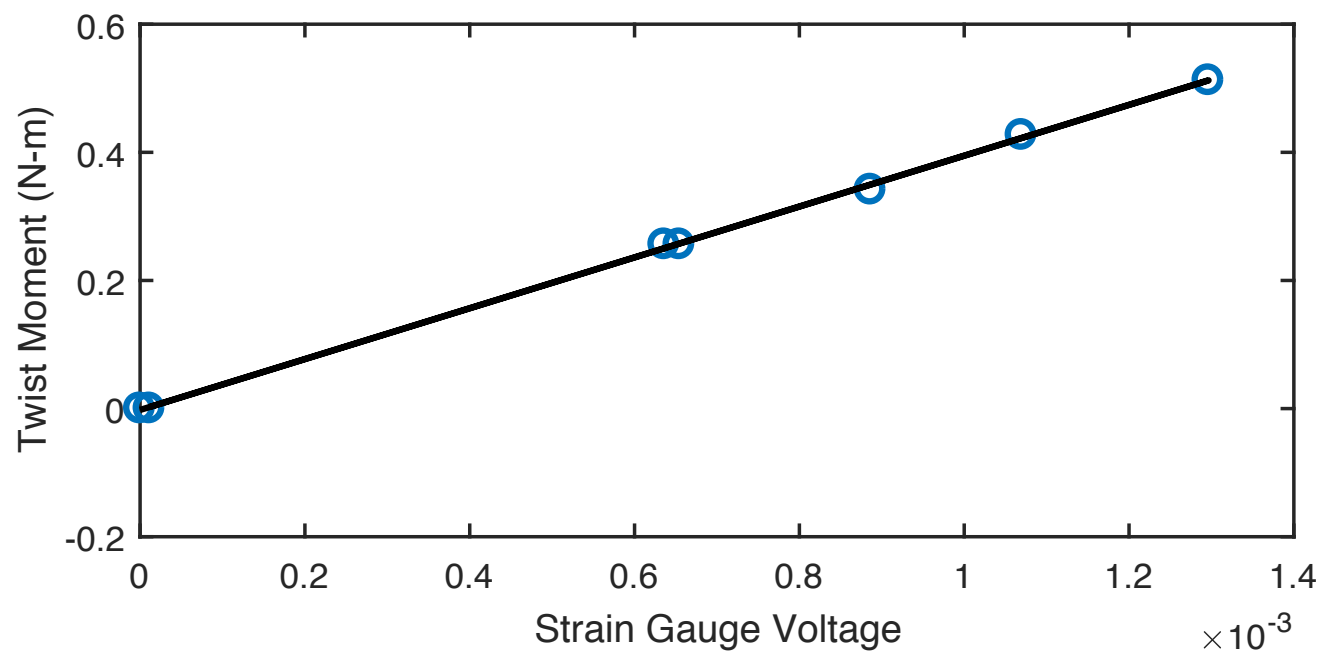

Figure 4.4: Measurement of strain gauge gain for twist moment. 


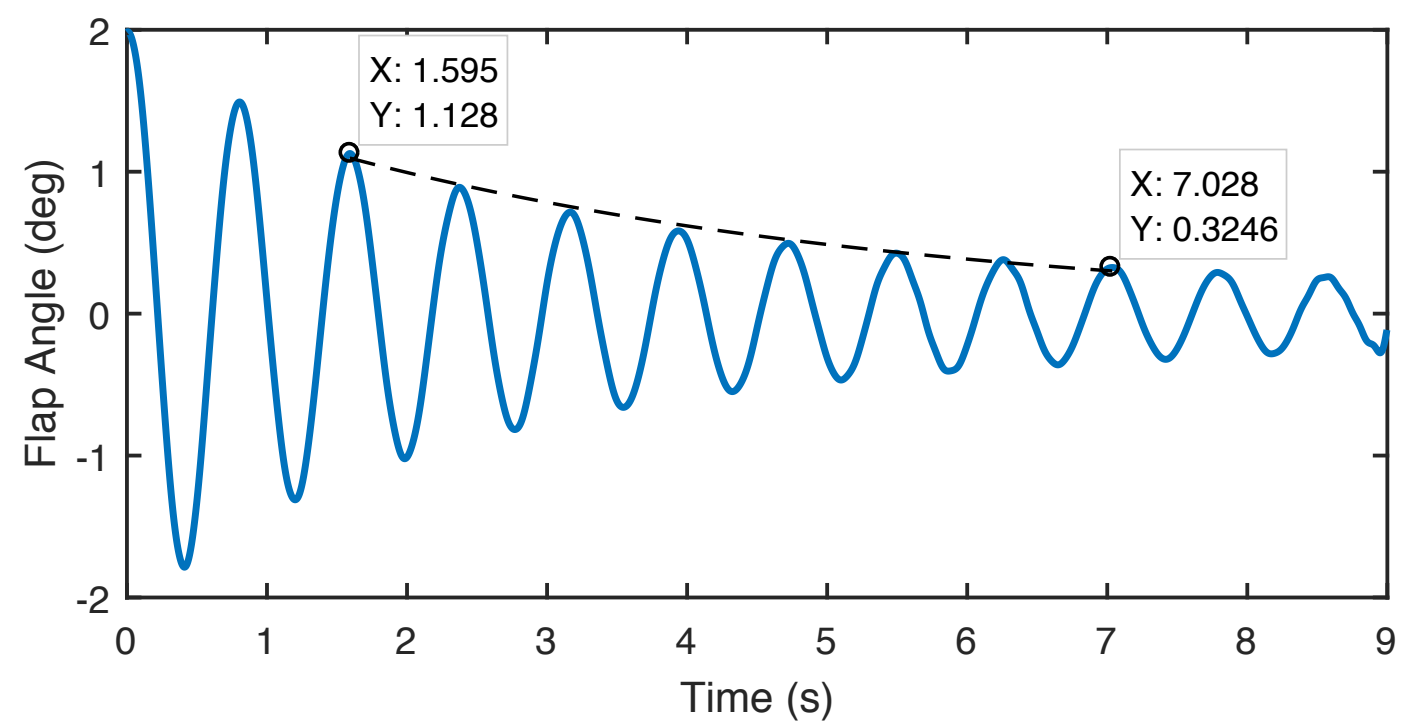

Figure 4.5: Impulse response of flap motion, as measured by the strain gauge. The dashed line shows the exponential decay from damping.

cient and rate gyro gains. The logarithm-decrement method was used to find the natural frequency and damping ratio, which was used in conjunction with the previously measured spring constant to calculate the moment of inertia. The natural frequencies were found to be $f_{n, \text { flap }}=1.29 \pm 0.01 \mathrm{~Hz}$ and $f_{n, t w i s t}=10 \pm 1 \mathrm{~Hz}$ and the damping coefficients, $\xi_{\text {flap }}=0.029 \pm 0.003$ and $\xi_{\text {twist }}=0.10 \pm 0.01$. From this, with the $K$ values from before, the moments of inertia can be calculated: $I_{\text {flap }}=1.13 \pm 0.03 \mathrm{~kg} \cdot \mathrm{m}^{2}$ and $I_{\text {twist }}=0.0020 \pm 0.0004 \mathrm{~kg} \cdot \mathrm{m}^{2}$.

Calibration of the pressure taps for unsteady pressure readings was performed using the method outlined in section 2.6. The model contains tubes of five different lengths of tubing: 24 in, 42 in, 60 in, 66 in, and 78 in. Pressure pulses were performed for each of the 28 pressure taps, but the tubing parameters proved similar enough for tubes of equal length that responses were averaged together each of the five groups. Figures 4.7-4.11 show the Bode plots for the transducer pressure divided by the reference pressure. For 


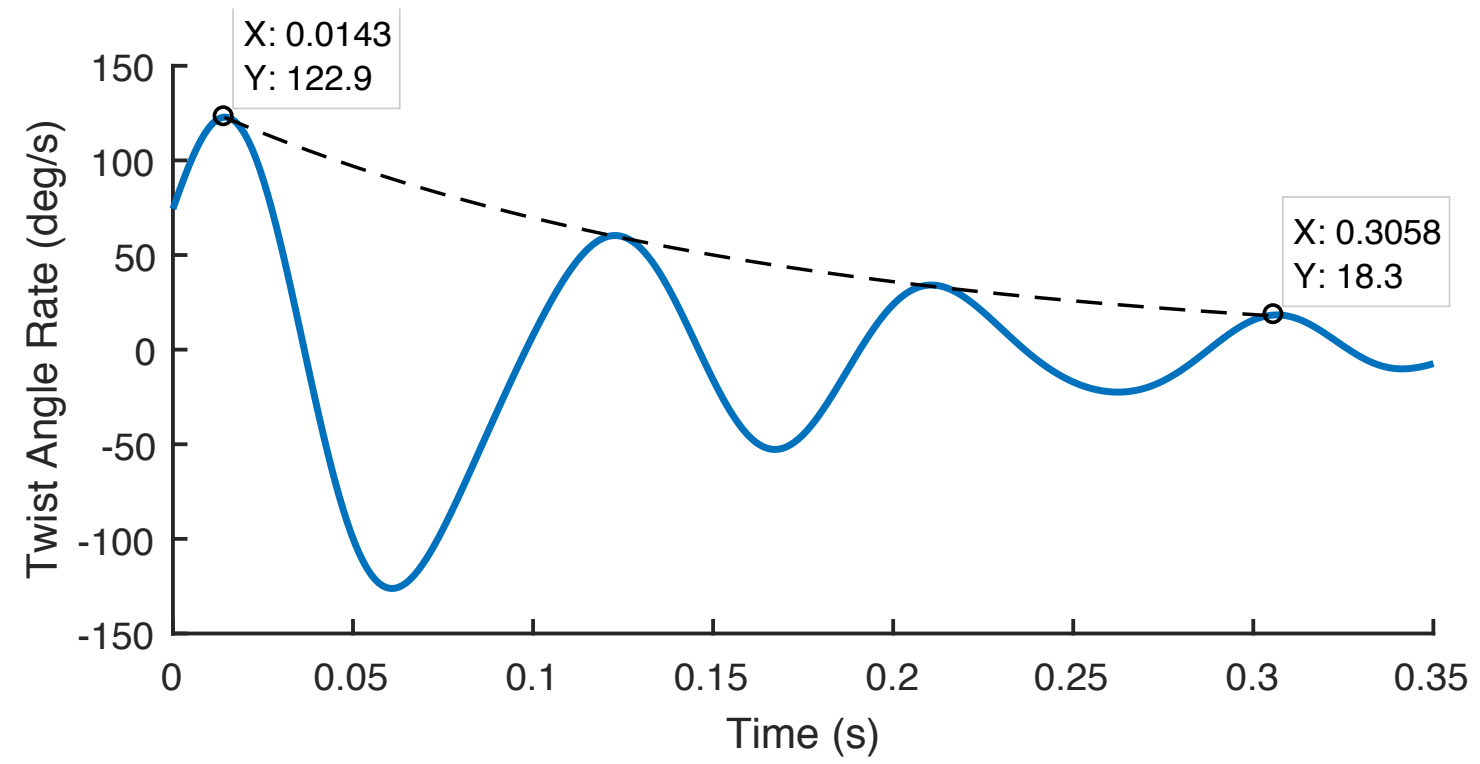

Figure 4.6: Impulse response of twist motion, as measured by the rate gyro. The dashed line shows the exponential decay from damping.

frequencies above $60 \mathrm{~Hz}$, the reference signal did not contain a sufficiently large signalto-noise ratio to be useful for a fit, so only frequencies below this were used to estimate tubing parameters; despite this, the transfer function appears to extrapolate beyond this frequency quite well. For the purposes of these experiments, validation up to $60 \mathrm{~Hz}$ is sufficient. Figures 4.12-4.16 compares the reference pressure to the pressure measured by the scanner and demonstrates the reconstruction of the reference pressure using the scanner measurement. All lengths of tube perform well at reconstructing, including the 78 inch tube despite a heavy time lag and attenuation.

\subsection{Static Pitch Sweep}

The first two wind-on runs consisted of static pitch sweeps to verify the installed angleof-attack of the model as it would have been impractical to attempt to install the model perfectly square to the mounting point of the turntable in the test section floor. The two runs were performed at $7 \mathrm{~m} / \mathrm{s}$ and $10 \mathrm{~m} / \mathrm{s}$. The pitch actuator motor was locked in position, 

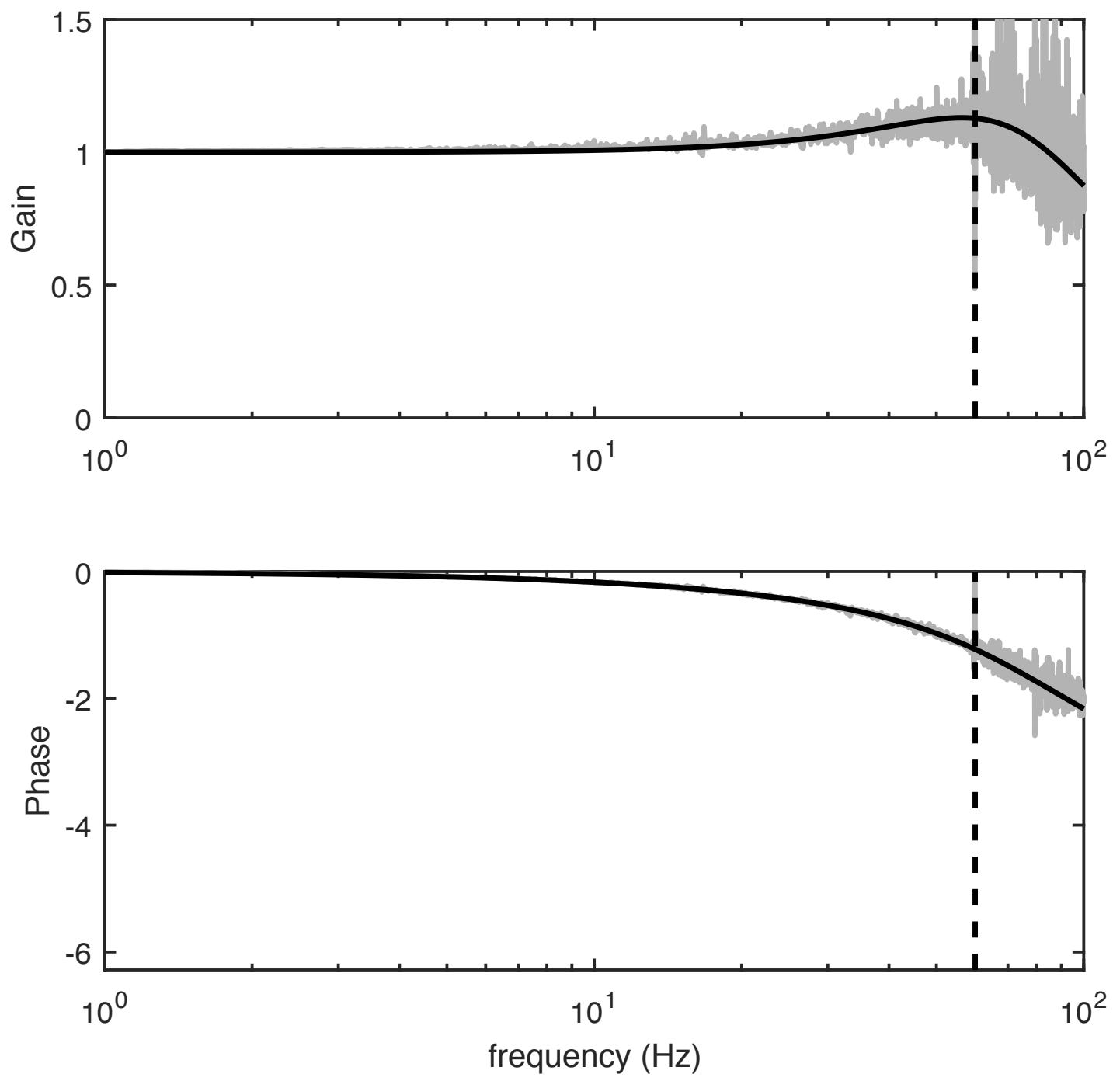

Figure 4.7: Bode plot for 24 inch tube. Below $60 \mathrm{~Hz}$, frequencies are actually amplified instead of attenuated. Transfer function was fit using only data left of the dashed line. 

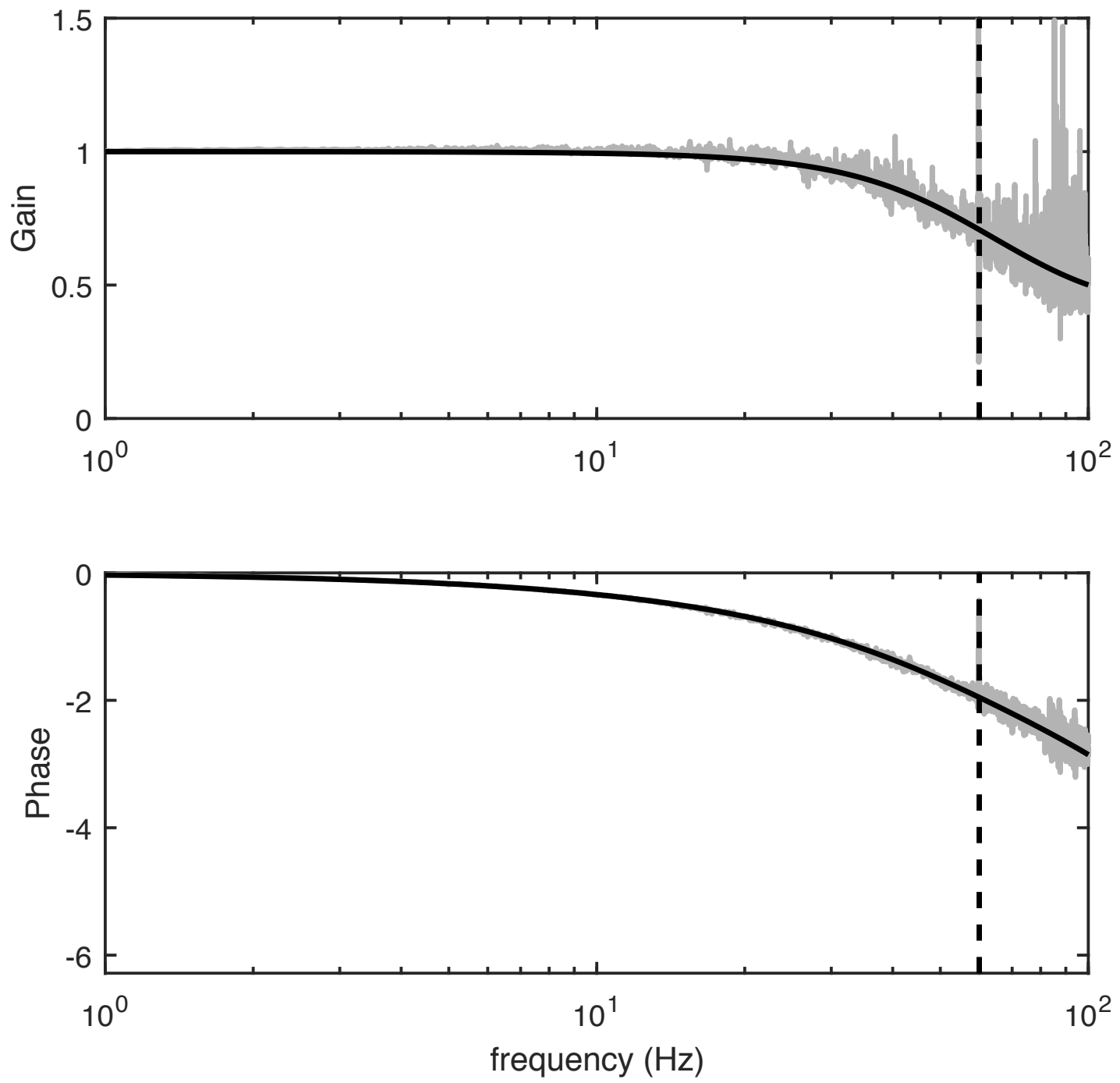

Figure 4.8: Bode plot for 42 inch tube. Transfer function was fit using only data left of the dashed line. 

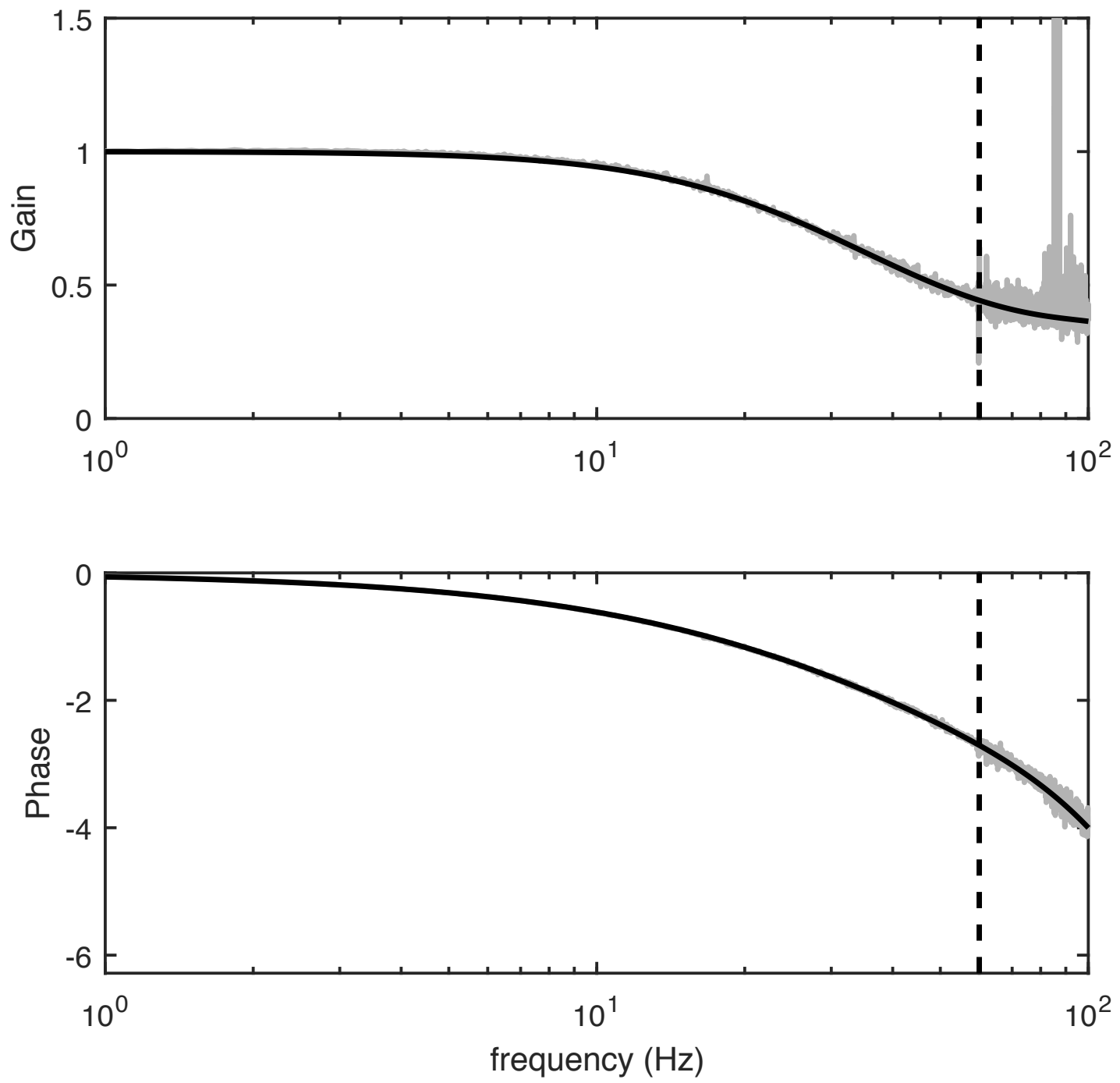

Figure 4.9: Bode plot for 60 inch tube. Transfer function was fit using only data left of the dashed line. 

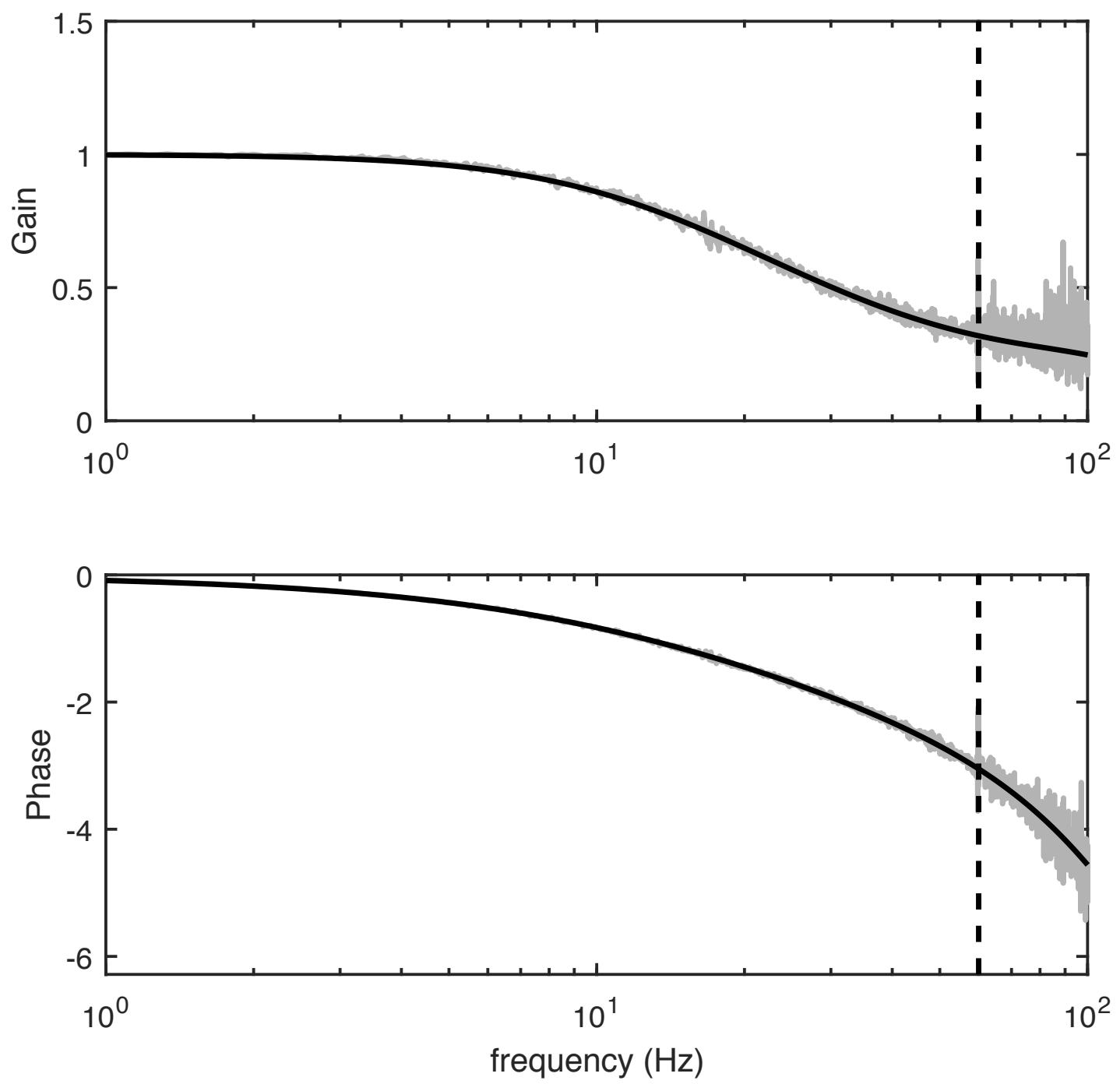

Figure 4.10: Bode plot for 66 inch tube. Transfer function was fit using only data left of the dashed line. 

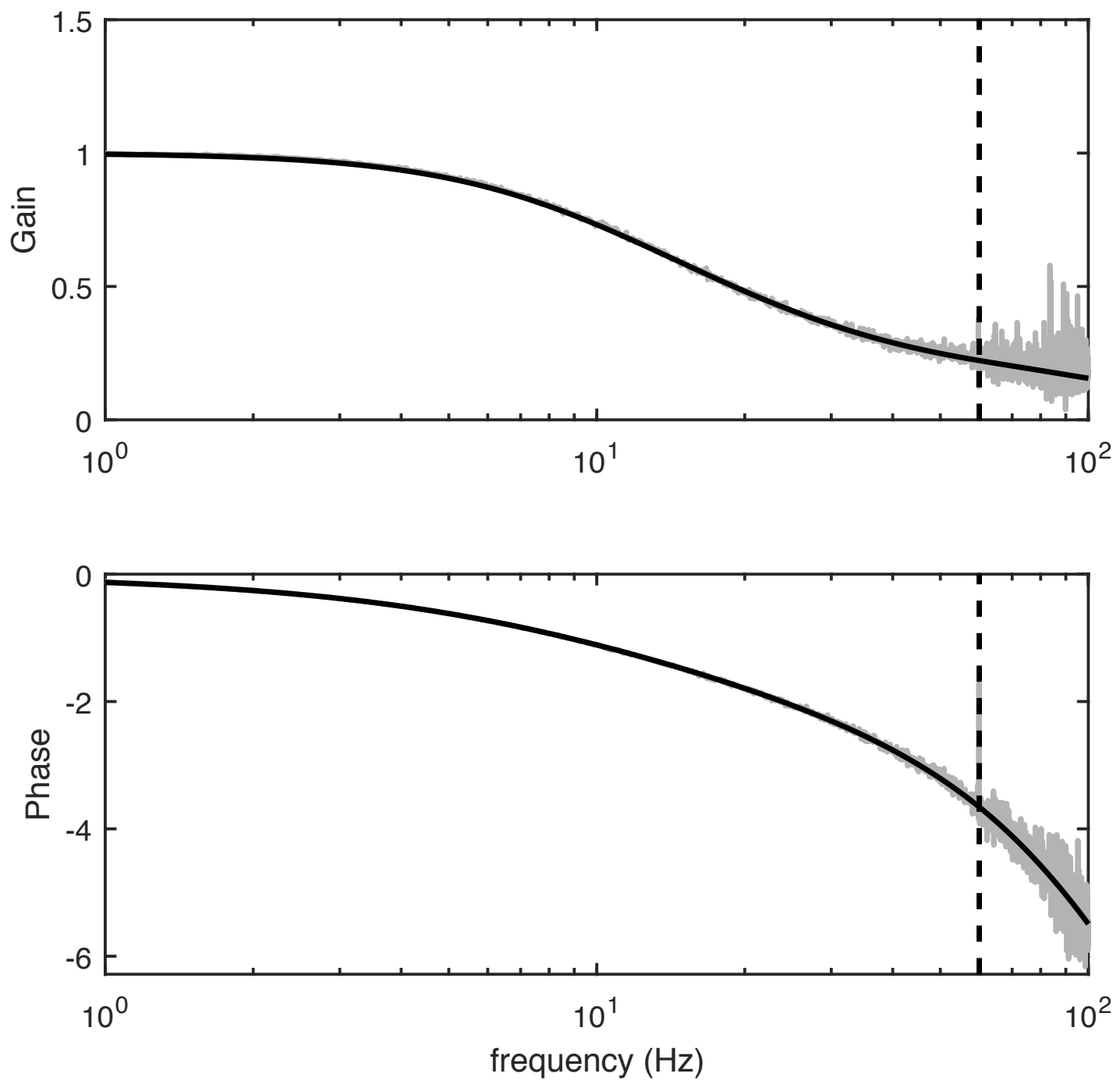

Figure 4.11: Bode plot for 78 inch tube. Transfer function was fit using only data left of the dashed line. 


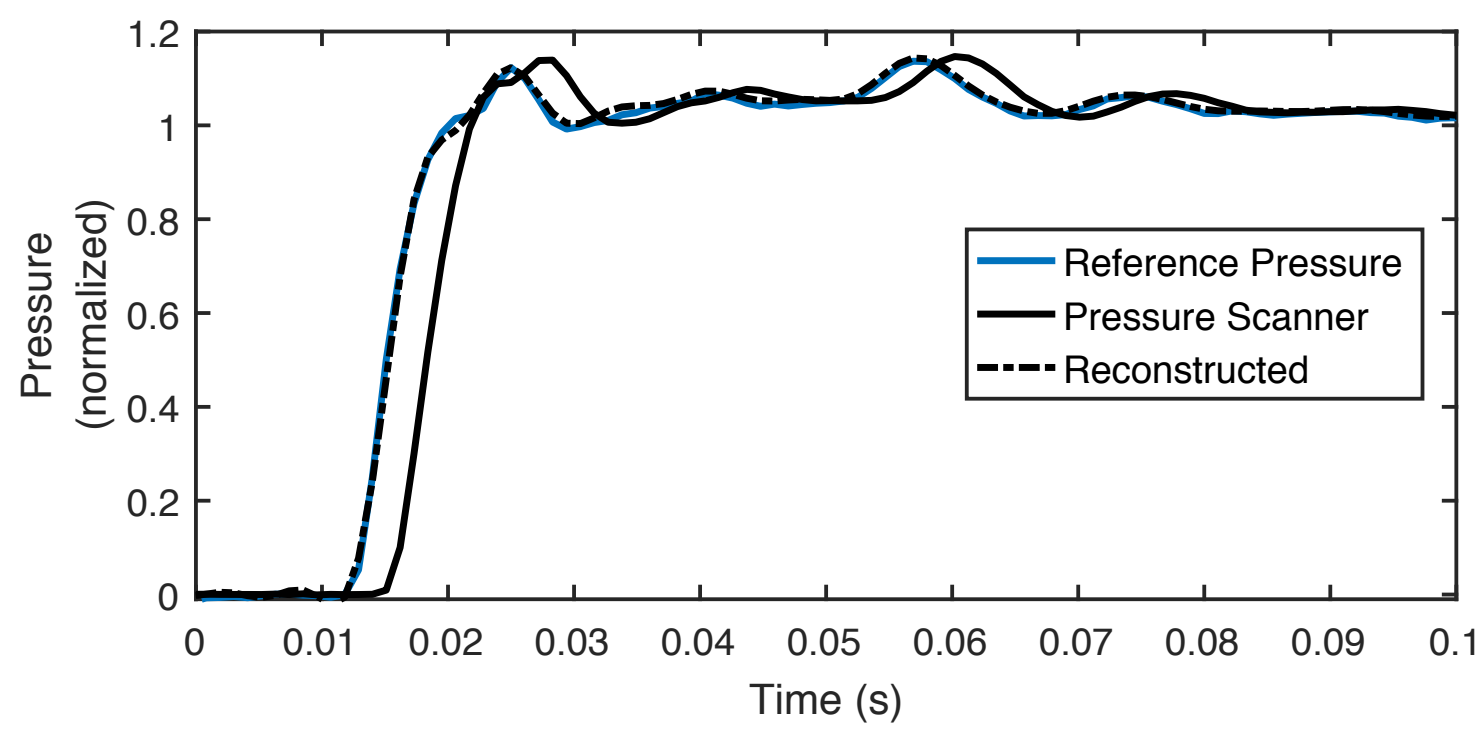

Figure 4.12: Reconstruction of reference signal for 24 inch tube.

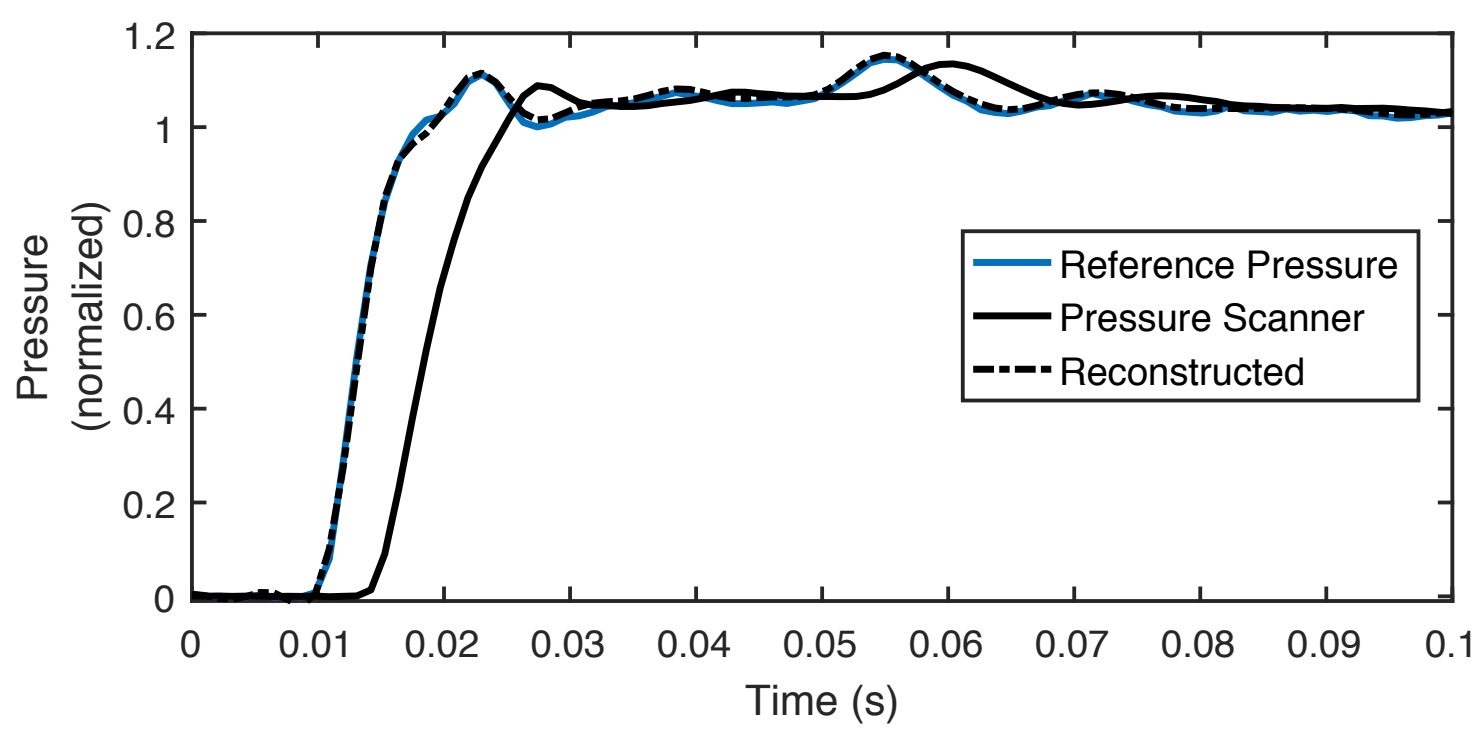

Figure 4.13: Reconstruction of reference signal for 42 inch tube. 


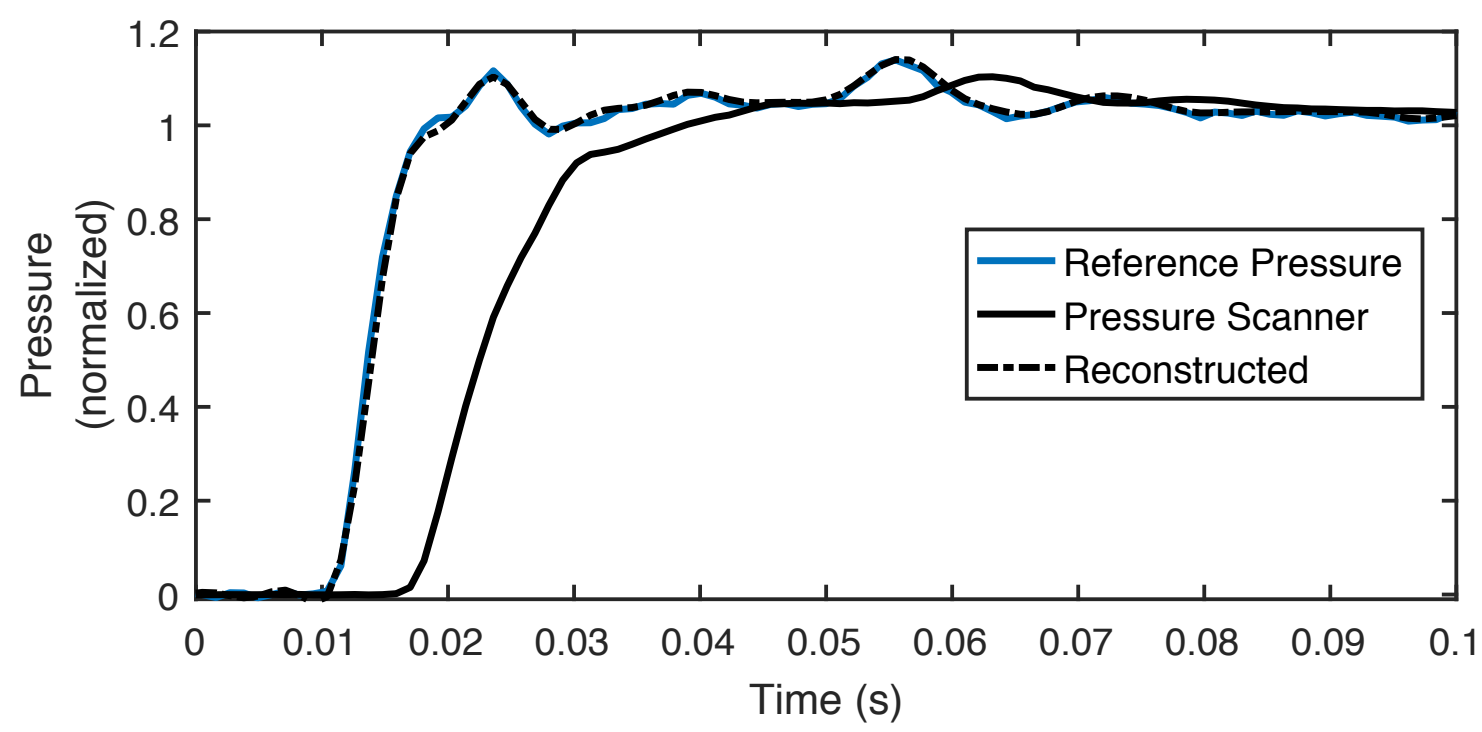

Figure 4.14: Reconstruction of reference signal for 60 inch tube.

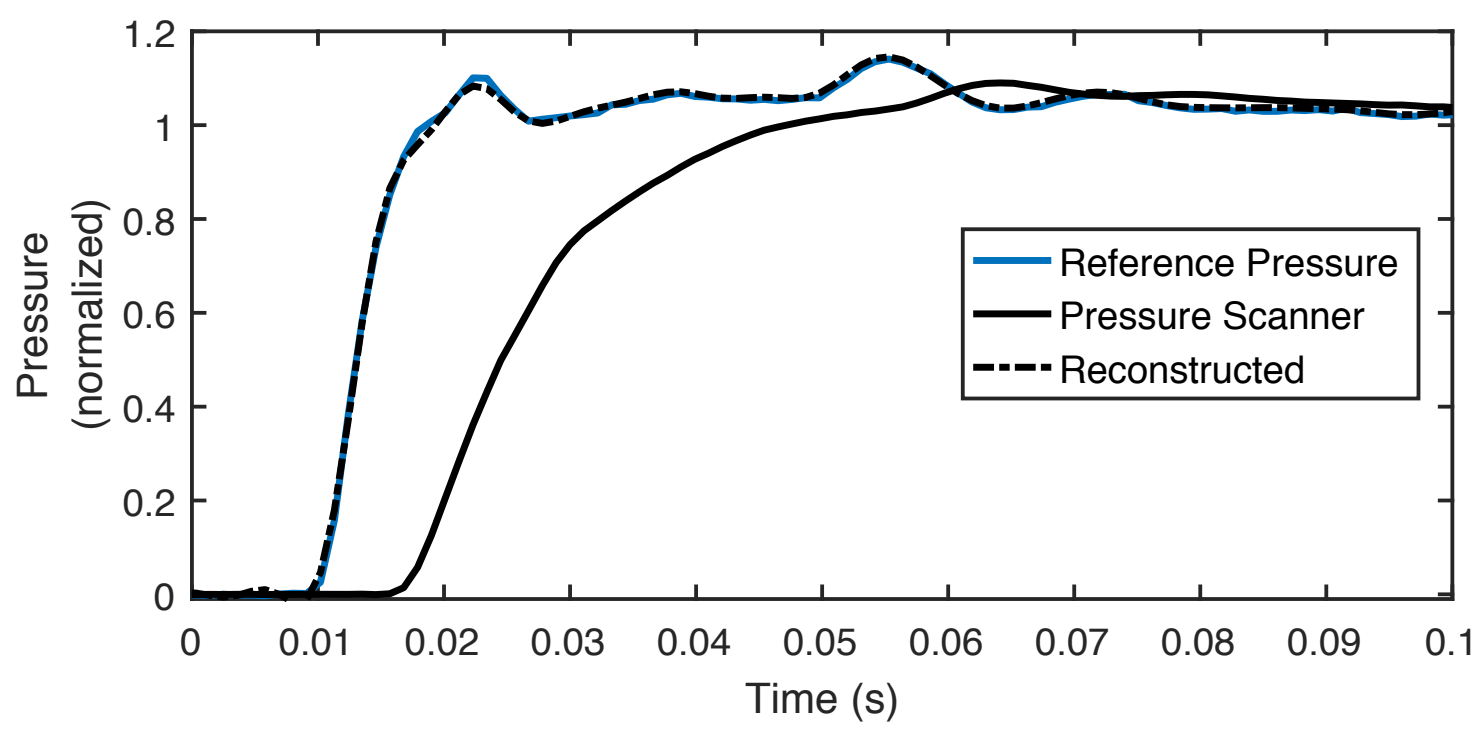

Figure 4.15: Reconstruction of reference signal for 66 inch tube. 


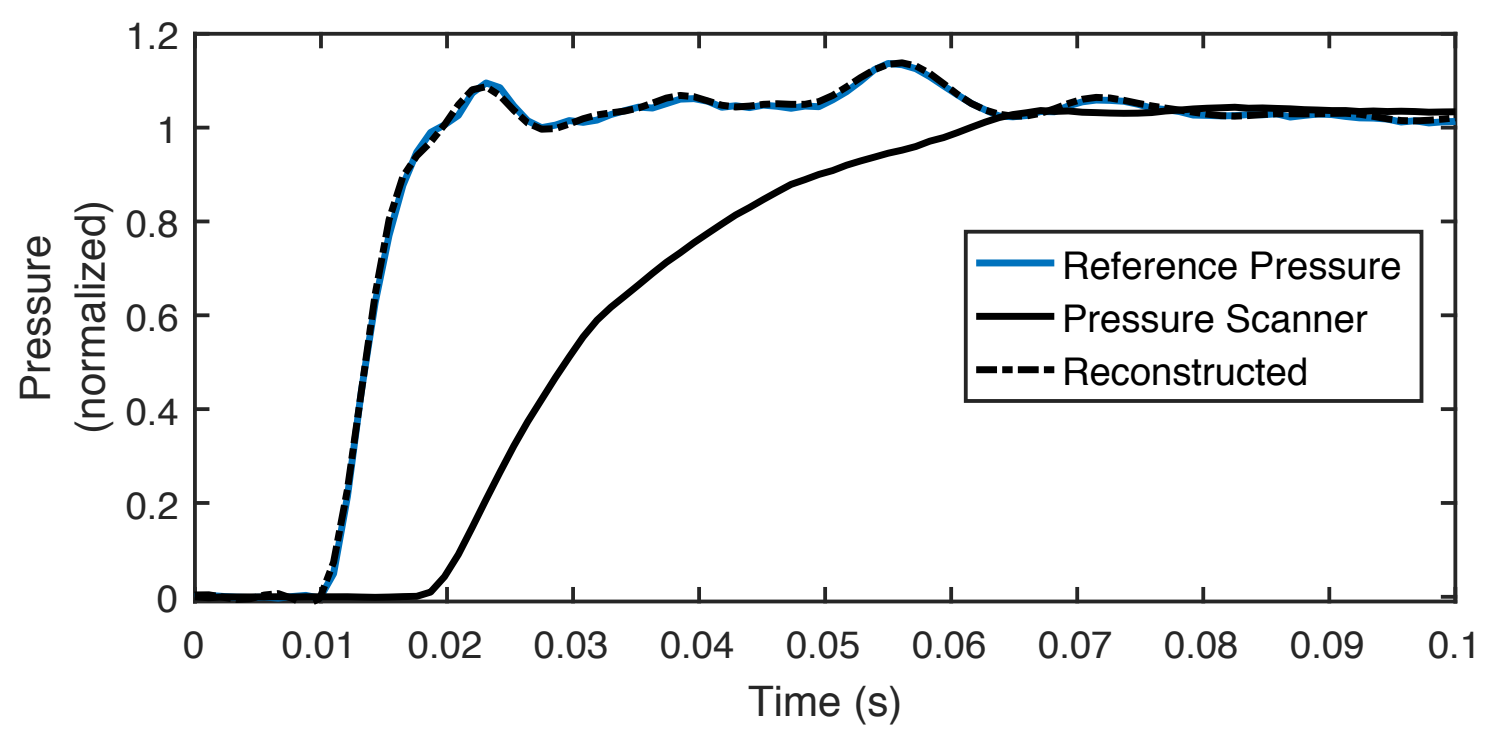

Figure 4.16: Reconstruction of reference signal for 78 inch tube.

and the turntable on the floor of the LSWT test section was rotated to set the pitch angle. The $7 \mathrm{~m} / \mathrm{s}$ run allowed for a much wider range of pitch angles as the $10 \mathrm{~m} / \mathrm{s}$ run caused dangerously excessive deflection when pitched above 4 degrees. Figures 4.17 and 4.18 show the load on the blade for tunnel velocities of $7 \mathrm{~m} / \mathrm{s}$ and $10 \mathrm{~m} / \mathrm{s}$ respectively. In these plots, the angle on the $\mathrm{x}$-axis is the turntable angle plus the elastic deflection of the twist degree of freedom measured using the strain gauge. The dashed line represents the lift predicted by steady lifting line theory. Note that the lift departs from the inviscid flow prediction at higher angles due to the low Reynolds number of these experiments. For $7 \mathrm{~m} / \mathrm{s}$, the chord Reynolds number, $R e_{c}$, ranges from 110,000 at the root, to 54,000 at the tip. For $10 \mathrm{~m} / \mathrm{s}, R e_{c}$ ranges from 150,000 at the root, to 77,000 at the tip.

This test was also used to verify the measurement of the sectional lift coefficient using the pressure taps. XFOIL was used to simulate the NACA $63_{3}-418$ with $R e_{c}=100,000$ and an $\mathrm{N}$-factor of 5. Figure 4.19 compares the simulation to the sectional lift at the center-span of the airfoil as measured by the pressure taps. In these plots, both sets of 


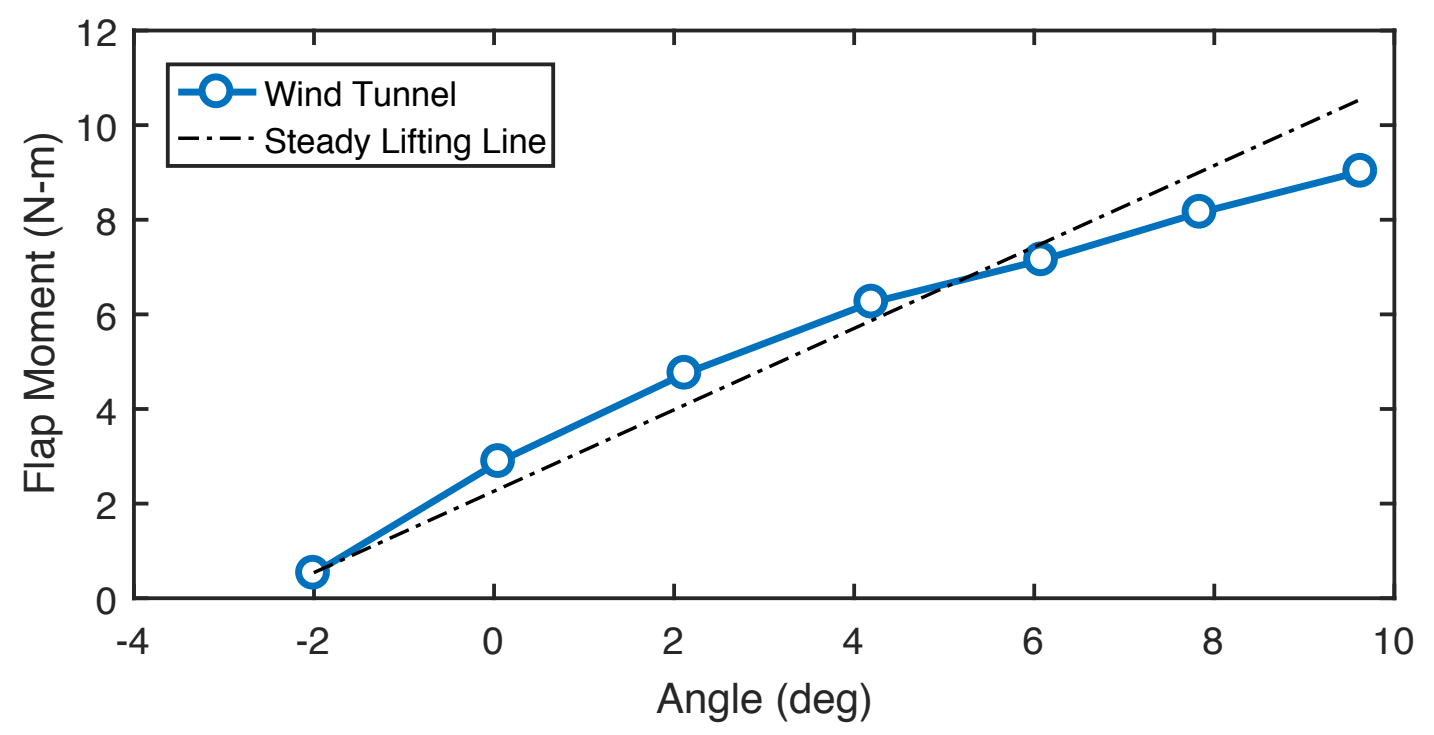

Figure 4.17: Static angle sweep at $7 \mathrm{~m} / \mathrm{s}$.

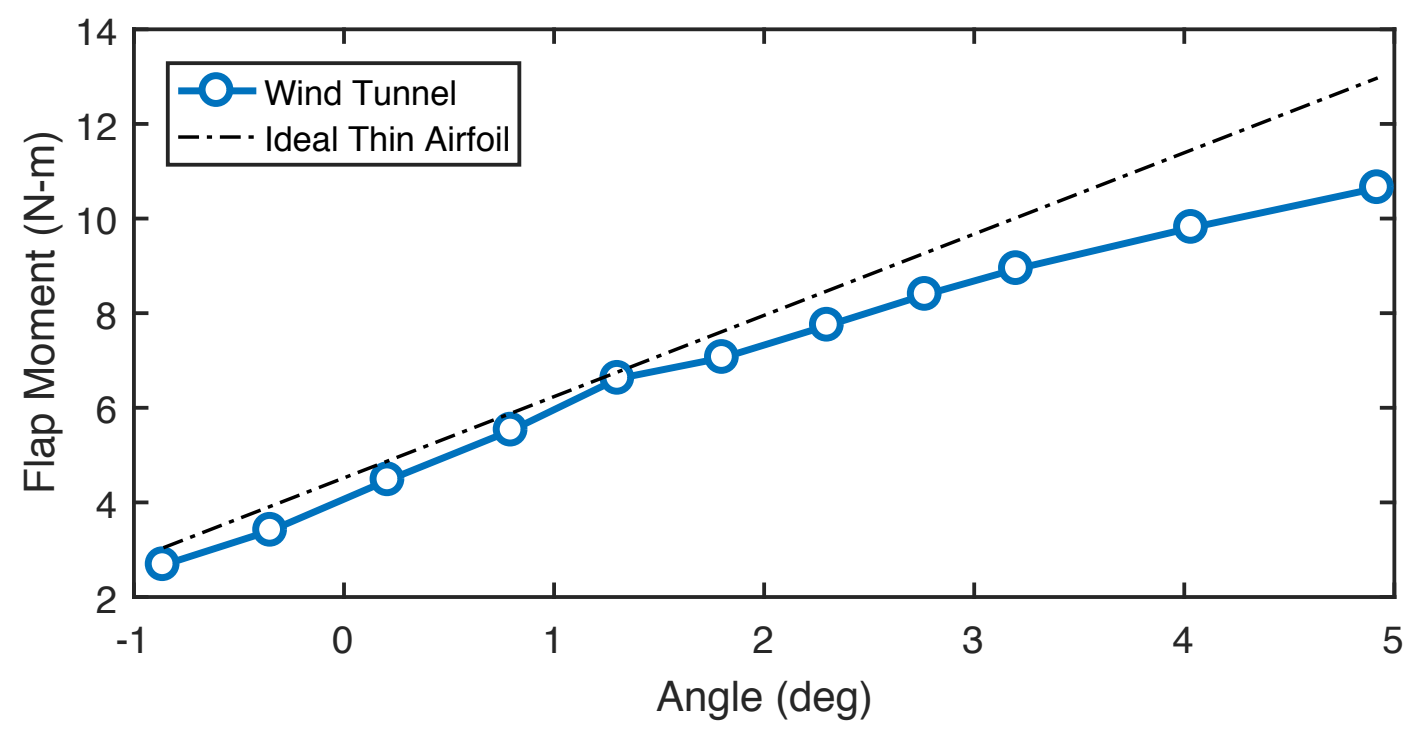

Figure 4.18: Static angle sweep at $10 \mathrm{~m} / \mathrm{s}$. 


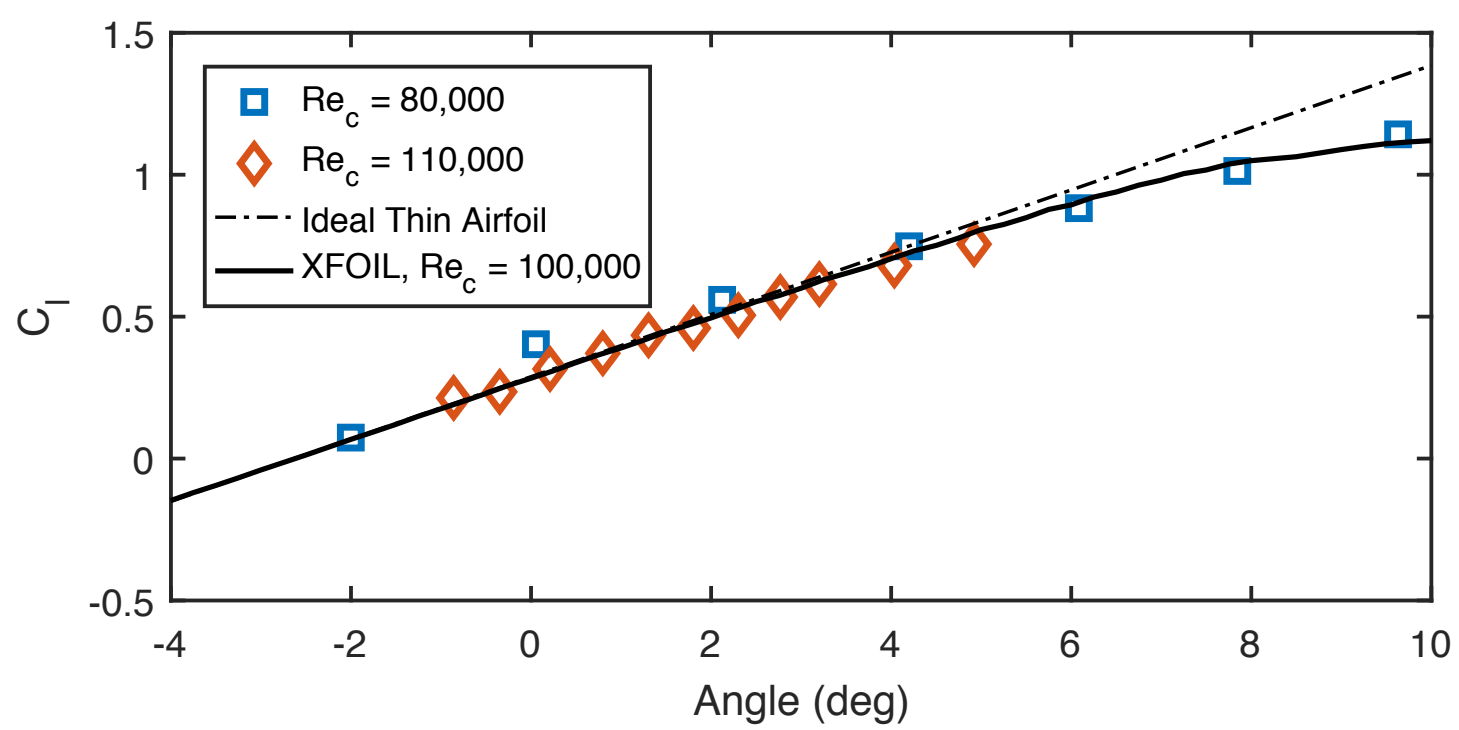

Figure 4.19: Static angle sweep.

experimental datapoints have been shifted up by a value of 0.19 . Due to the sparse distribution of pressure taps around the center of the blade, the numerical integration poorly measures the lift. However, this error appears to be a constant bias as the overall trend seems to match the simulation. Though the total value of the lift measured by the pressure taps cannot be trusted, relative changes can be accurately measured. This is particularly good for this study as it's primarily concerned with the unsteady component of lift.

\subsection{Pitch Oscillations Tests}

The primary goal of the wind tunnel experiments is to subject the blade to aerodynamic disturbances and measure the response loads and motion. These are compared to the output of the simulation model. The aerodynamic disturbances are applied by a pitch oscillation motor at the cantilevered end of the elastic base. The pitch motor is capable of driving oscillations from around $1 \mathrm{~Hz}$ to $7 \mathrm{~Hz}$. Frequency sweeps of the pitch motor were performed at $0^{\circ}$ mean angle of attack at $7 \mathrm{~m} / \mathrm{s}$ and $10 \mathrm{~m} / \mathrm{s}$ freestream velocity. Additionally, 
3 different oscillation amplitudes, $A_{\phi_{t}}$ were used: $\pm 1^{\circ}, \pm 2^{\circ}$, and $\pm 4^{\circ}$. A range of reduced frequencies based on the chord at mid-span, $k_{c}$, were tested from 0.13 to 0.56 . Because the reduced frequency is dependent on semi-chord, $b$, the reduced frequency for each test case is reported as $k_{c}$, the reduced frequency at the center-span. The actual reduced frequency varies linearly over the blade from $1.32 k_{c}$ at the root and $0.68 k_{c}$ at the tip. For reference, $k$ values less than 0.05 are typically assumed to be quasi-steady, and values above 0.2 are considered highly unsteady.

For each frequency, velocity, and amplitude, 11 seconds of data were recorded. The measurements were phase-lock averaged to obtain a smooth curve representing a single period of oscillation. Figure 4.20 shows raw data for the twisting motion of the blade, as measured by the rate gyro, for $k_{c}=0.098, V=10 \mathrm{~m} / \mathrm{s}$, and $A_{\phi_{t}}=2^{\circ}$. In addition to the phase-lock averaging, each signal is also smoothed using a Fourier transform. All harmonics with an amplitude less than 0.01 times the fundamental are eliminated.

\subsubsection{Baseline Case}

This section presents data in detail for the case of $k_{c}=0.098, V=10 \mathrm{~m} / \mathrm{s}$, and $A_{\phi_{t}}=2^{\circ}$. This case was chosen for its moderate oscillation amplitude and low frequency. This frequency is near the natural frequency of the blade, $k_{c}=0.066$, so large deflections can be measured.

Figure 4.22 shows the measured twist motion for this baseline case. The strain gauge and the rate gyro do not match, but this is expected. The strain gauge has no knowledge of the pitch actuator and only measures the elastic component of the twist, while the rate gyro reads the total angle. The commanded twist angle can be inferred by subtracting the elastic twist, measured by the strain gauge, from the total twist, measured by the rate gyro. In a simulation which predicts elastic twist response, this twist command would serve as input. However, for reasons mentioned in section 3.3, the simulation model used in this 


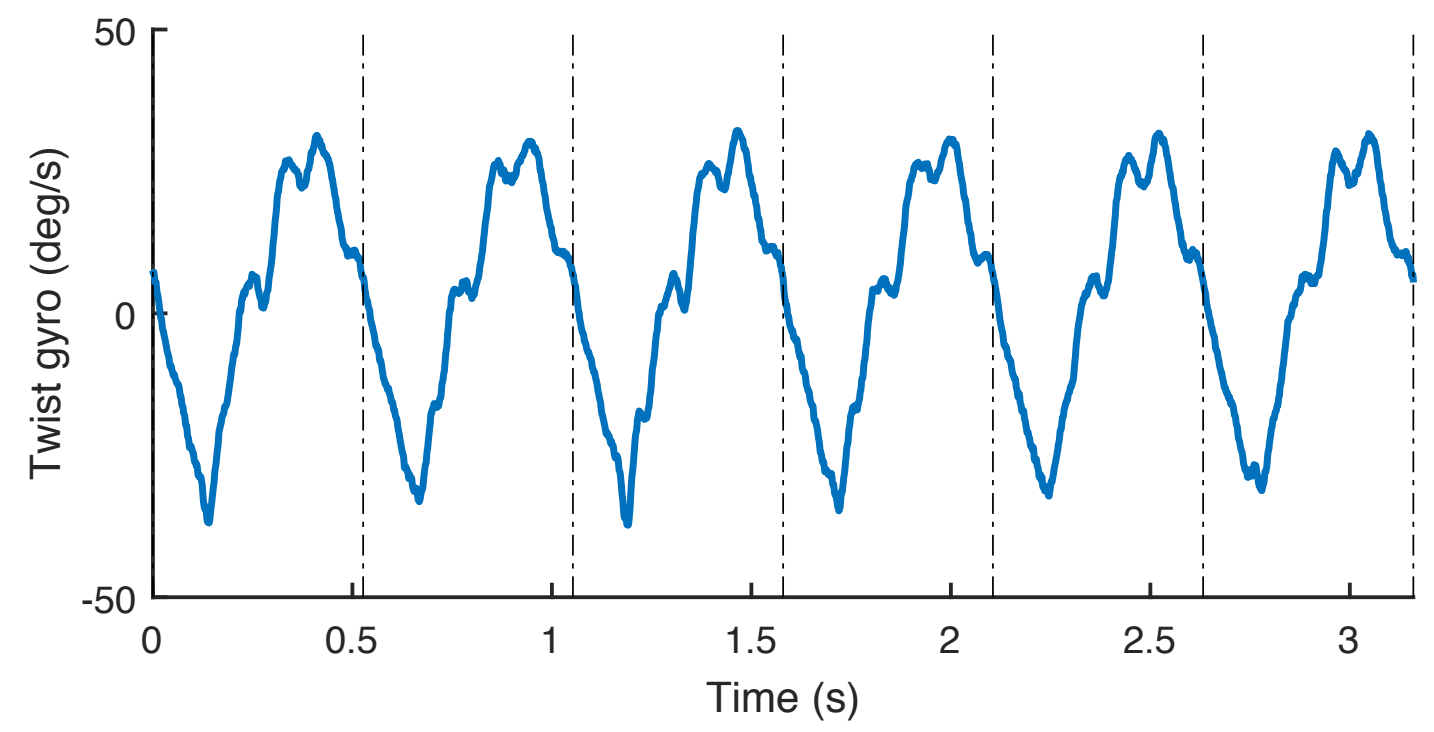

Figure 4.20: Rate gyroscope measurement of twisting motion for $k_{c}=0.098, V=10 \mathrm{~m} / \mathrm{s}$, and $A_{\phi_{t}}=2^{\circ}$. The dashed lines represent the windows of each period to be averaged. The first 6 of 20 windows are shown here.

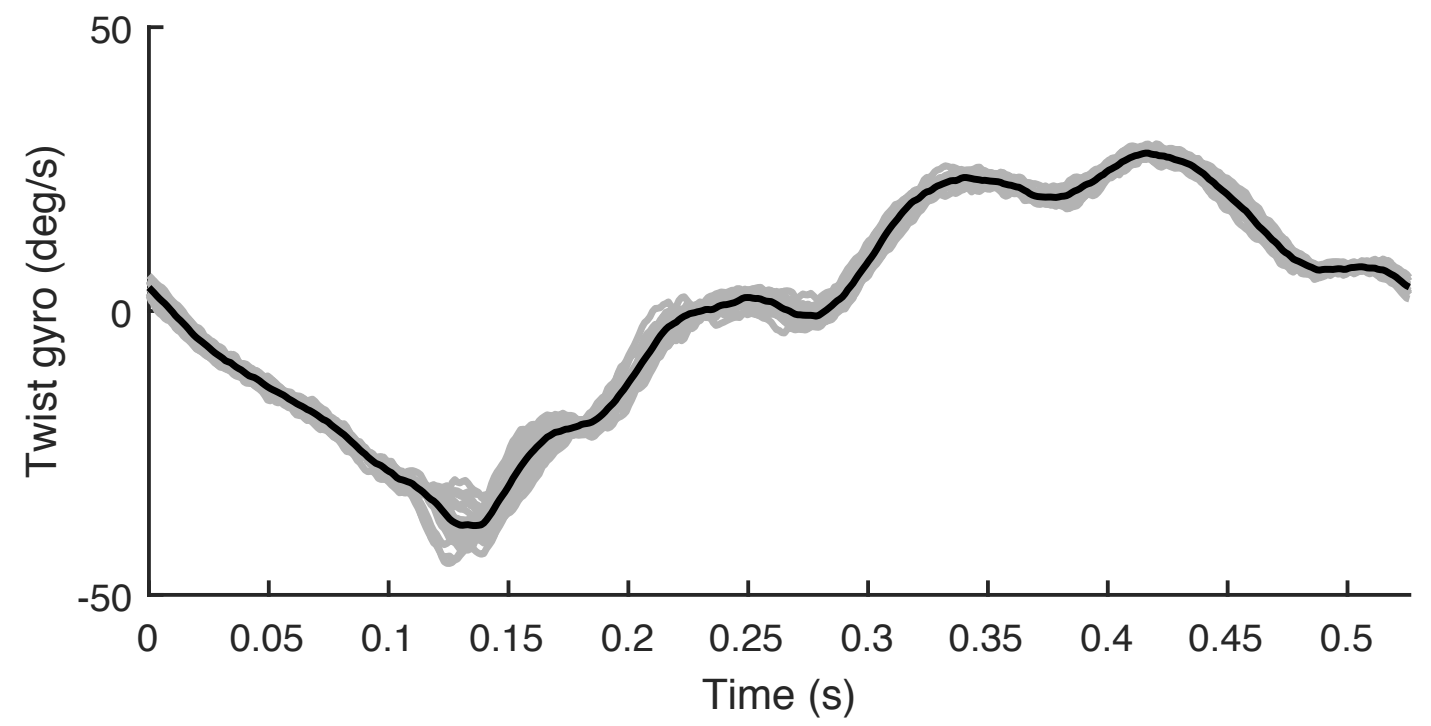

Figure 4.21: Each of the 20 windows from Figure 4.20, shown in grey, stacked on top of each other. The phase-lock average is shown in black. 


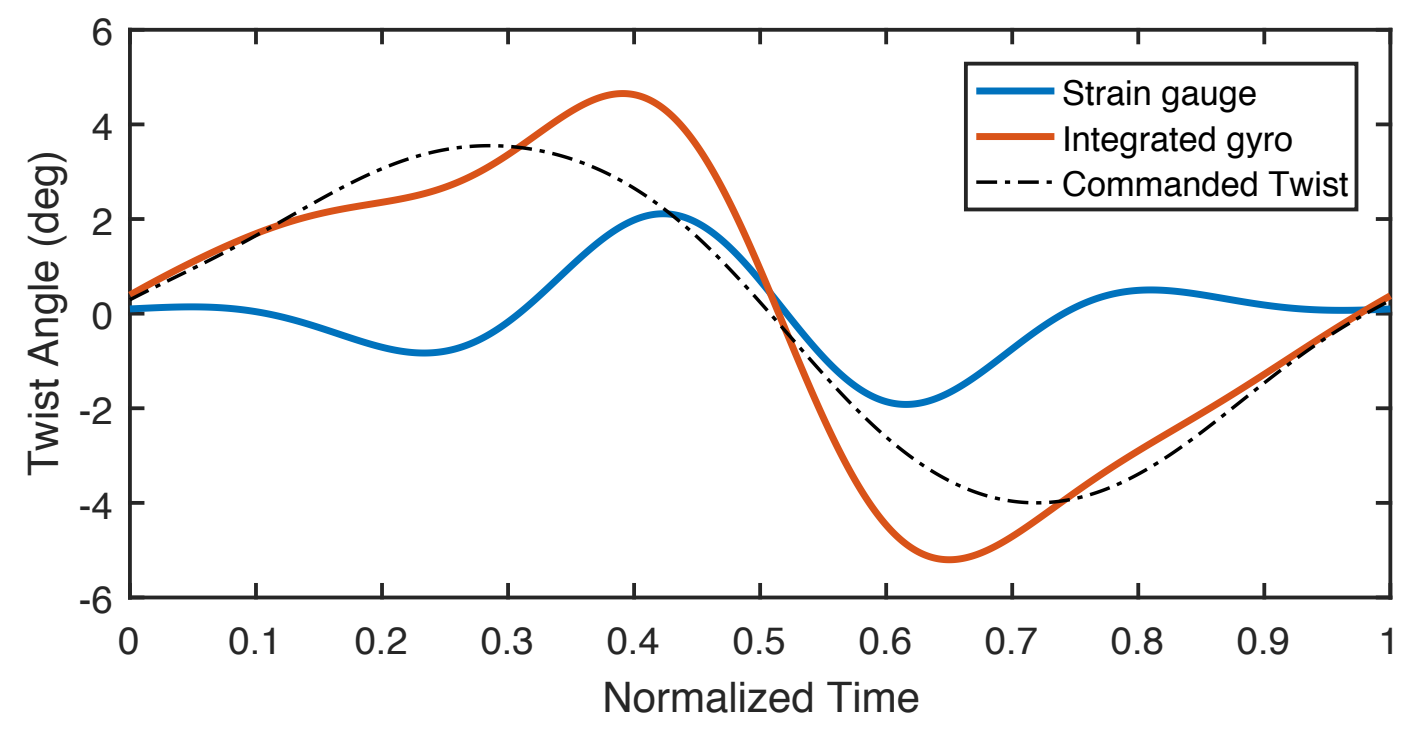

Figure 4.22: Twist motion measurements for the baseline case, $k_{c}=0.098, V=10 \mathrm{~m} / \mathrm{s}$, and $A_{\phi_{t}}=2^{\circ}$. The total twist is used as the input to the simulation model.

work only predicts flap response. The total twist motion, measure by the rate gyro, is used as input to the simulation model.

The flap response and moment for the baseline case are shown in Figure 4.23, with the total twist motion at the bottom. The deflection output would serve as input to fatigue prediction models, and is the most important output variable for the simulation. The flap response matches well, except for a slight phase lag.

Figure 4.24 shows the sectional lift at the center span as measured by the pressure taps and as predicted by the simulation. This matched very well. Surprisingly, the sectional lift measured at the additional spanwise locations, shown in Figure 4.25, also matches closely with the simulations, despite only using two pressure ports to calculate the lift at these locations. There is one exception; the lift measured by the root section of the blade underestimates the lift predicted by the simulation. The discrepancy likely results from the sizable gap between the root and the floor, as well as the uncovered hole where 

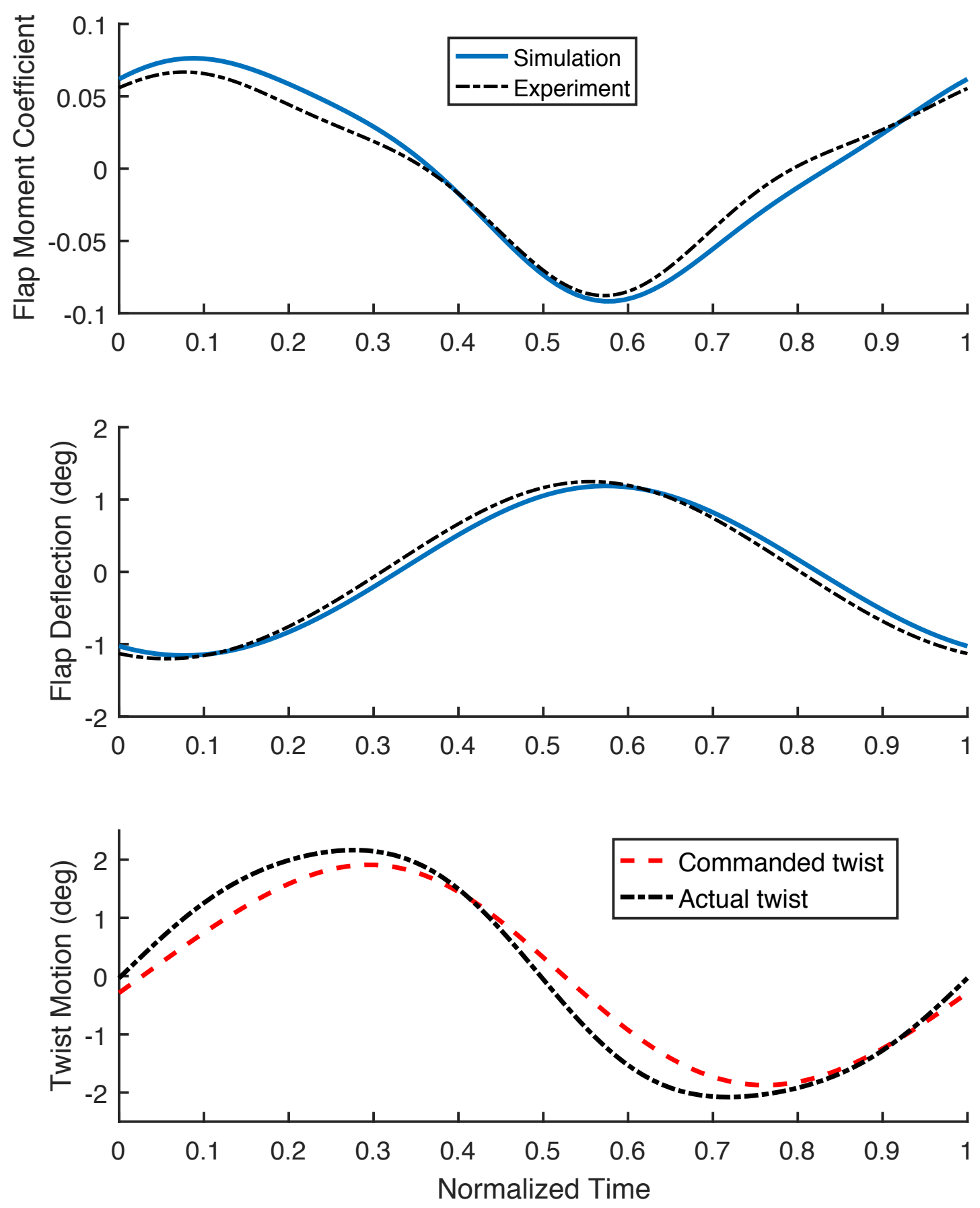

Figure 4.23: Simulated flap response and moment for the baseline case, $k_{c}=0.098$, $V=10 \mathrm{~m} / \mathrm{s}$, and $A_{\phi_{t}}=2^{\circ}$, compared to those measured by the experiment. The measured twist motion, used as input to the simulation model, is shown at the bottom. 


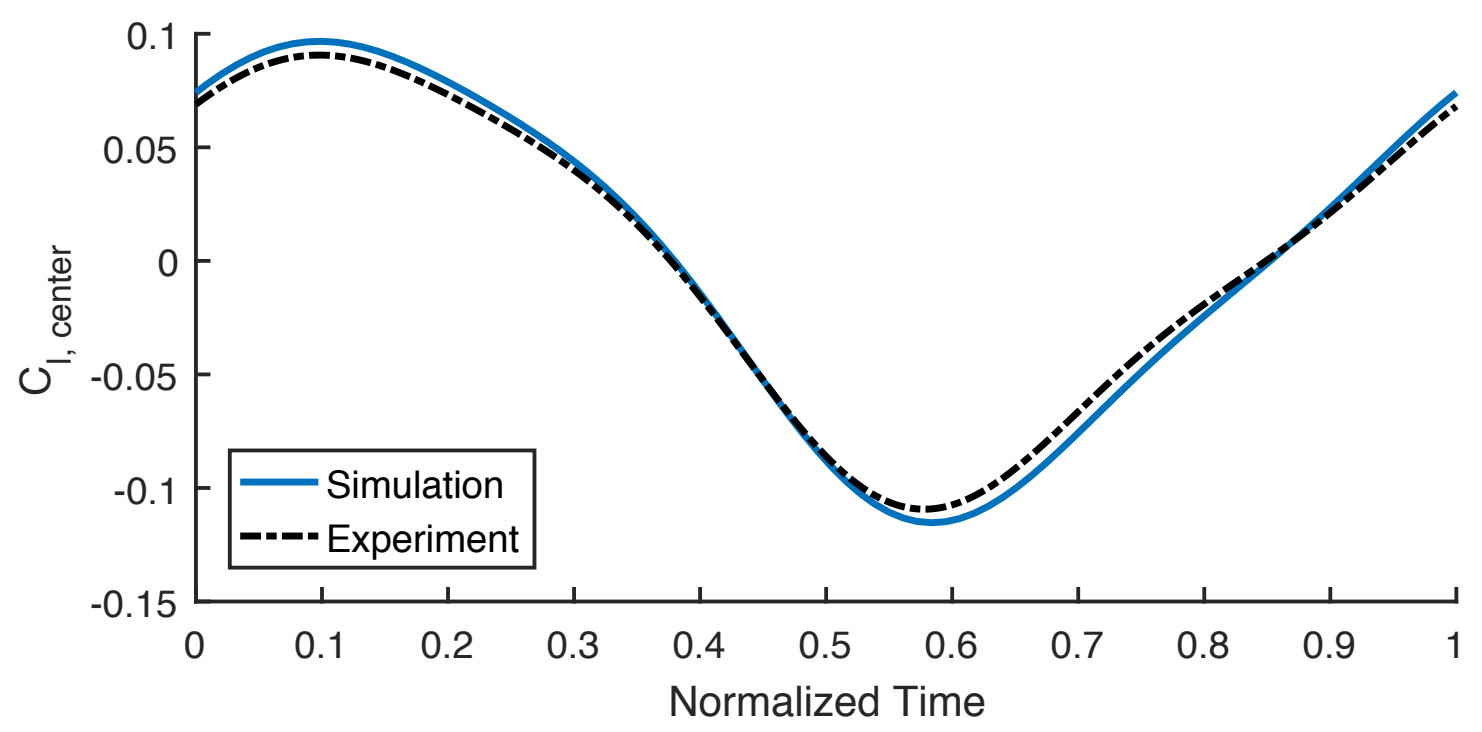

Figure 4.24: Center-span lift coefficient for the baseline case, $k_{c}=0.098, V=10 \mathrm{~m} / \mathrm{s}$, and $A_{\phi_{t}}=2^{\circ}$.

the supports enter the floor allowing air to flow around the root from the pressure side to suction side, and partially reducing the lift near the root.

The simulated twist moment is shown in Figure 4.26. This figure is included to demonstrate that the elastic twist response cannot be predicted. The design of the bearing blocks, for preventing edgewise motion, cause a much higher twisting moment at the elastic base than is predicted by the model. Besides this discrepancy and the $c_{l}$ discrepancy at $2 \%$ chord, both of which can be attributed to the design of the test platform, the match between the theoretical model developed in Chapter 3 and the data is very compelling.

\subsubsection{Effect of Reduced Frequency}

This section compares the relative performance of the simulation as reduced frequency is increased. All cases discussed in this section have a nominal twist amplitude, $A_{\phi_{t}}$, of $2^{\circ}$.

Figures 4.27-4.30 compare measurements of flap deflection and moment to the the- 

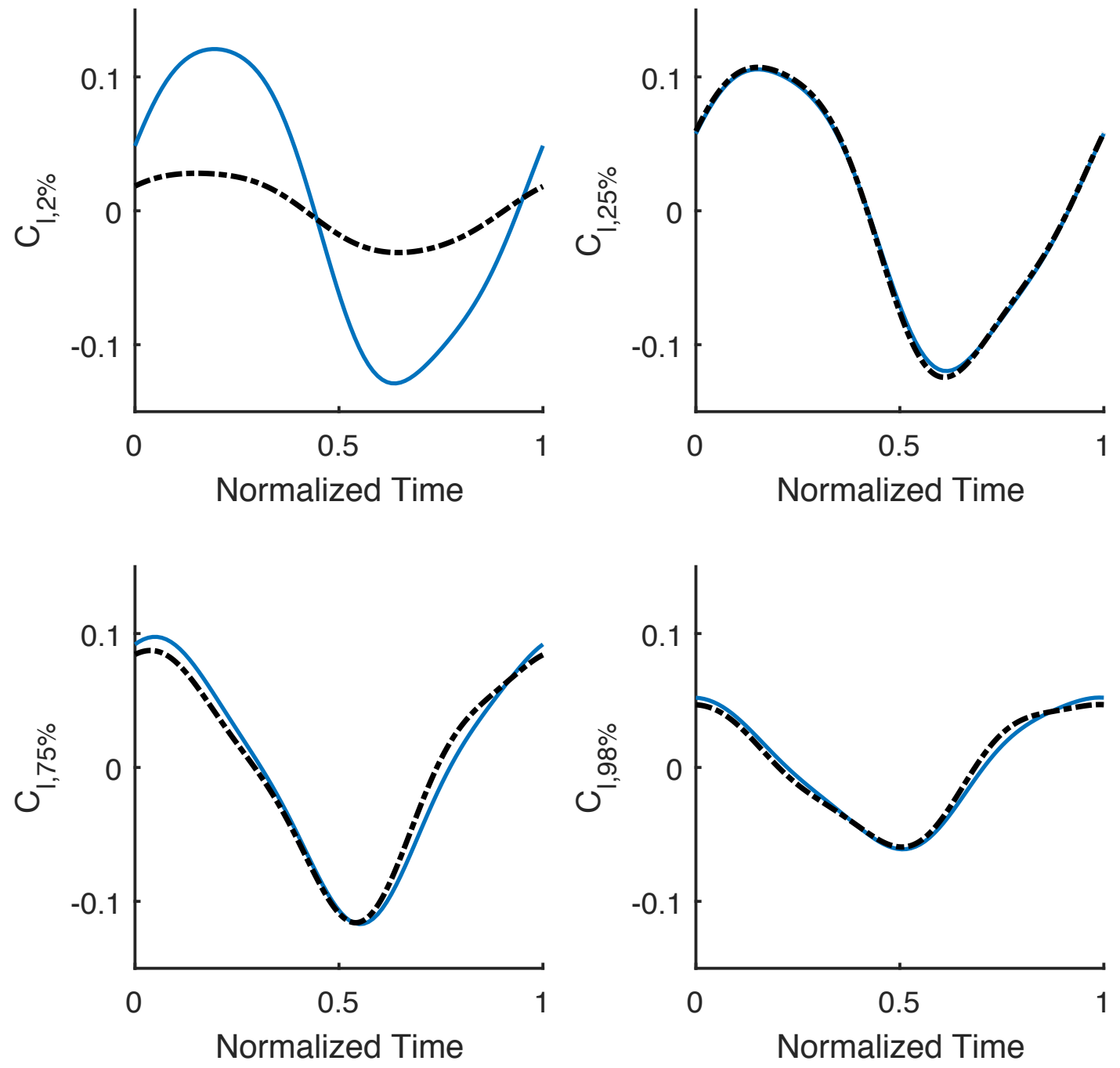

Figure 4.25: Simulated lift coefficients at various span locations for the baseline case, $k_{c}=0.098, V=10 \mathrm{~m} / \mathrm{s}$, and $A_{\phi_{t}}=2^{\circ}$. 


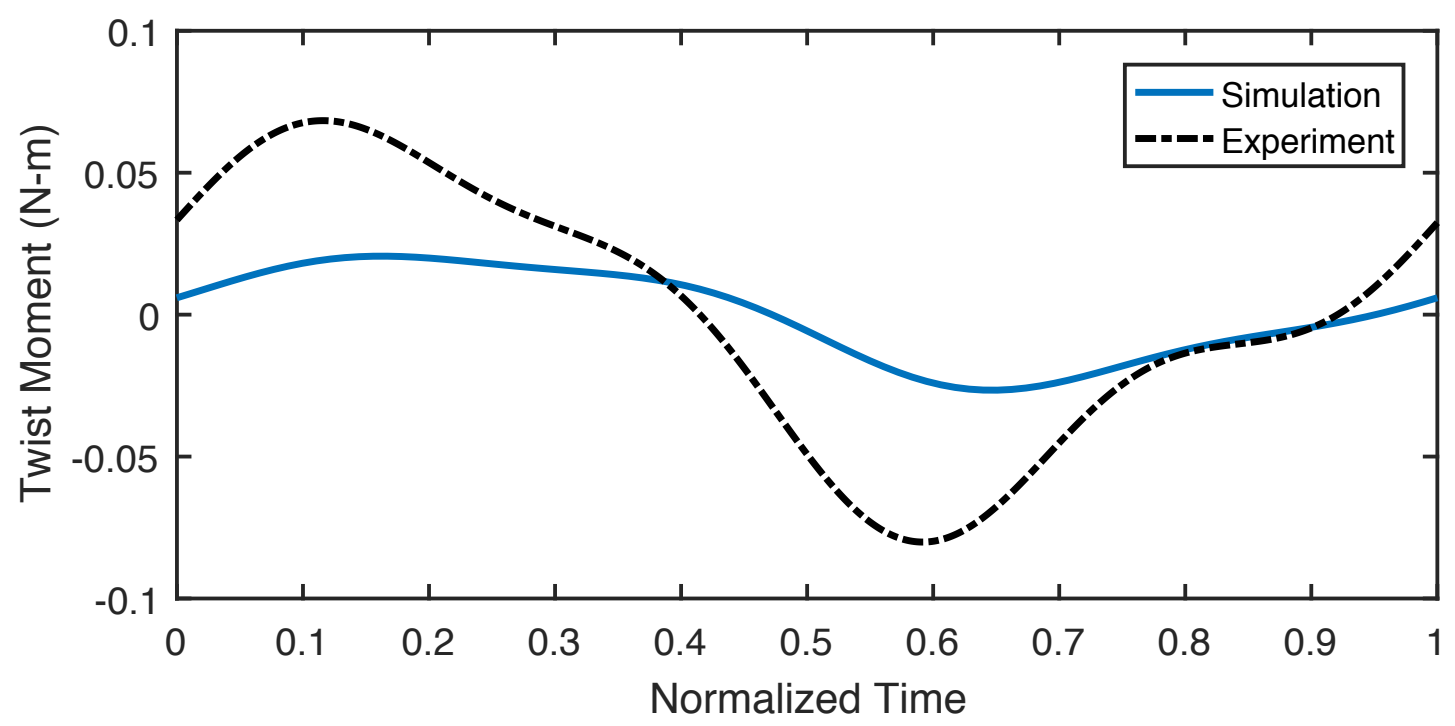

Figure 4.26: Simulated twist moment for the baseline case, $k_{c}=0.098, V=10 \mathrm{~m} / \mathrm{s}$, and $A_{\phi_{t}}=2^{\circ}$, compared to that measured by the experiment.

oretical model as $k_{c}$ increases. In Figure $4.27, k_{c}=0.13$, as with the baseline case, Figure $4.23, k_{c}=0.098$, there is a slight phase lag in the flap response. There is a some disagreement between the measured and simulated flap moment, but this does not appear to affect the quality of the flap deflection. In Figure 4.28, the simulated deflection is the correct shape and amplitude, but is phase shifted. It is unknown what is causing this error, but this likely would not negatively affect fatigue simulations. As the frequency grows larger, the resulting flap motion becomes smaller, and the accuracy of deflection prediction at these frequencies becomes less important. Additionally, as the flap deflection becomes smaller, the confidence in the experimental measurement of deflection decreases; errors such as loose fit between blade and base and bending of the assumed-rigid carbon-fiber tubes may explain the discrepancy. In Figures 4.29 and $4.30, k_{c}=0.31$ and 0.39 respectively, the phase shift of the simulated deflection becomes extreme, but with flap motion on the order of hundredths of a degree, the experimental measurement of deflection and load 
cannot be trusted. These frequencies are higher than the design range for the experimental apparatus and higher than is expected for large wind turbines.

Except for the $2 \%$ span location, the sectional lift coefficient from the pressure taps matched with the simulation for nearly all frequencies, amplitudes, freestream velocities, and frequencies, even up to $k_{c}=0.56$. Figures $4.31-4.34$ show the sectional lift at the center span as measured by the pressure taps and as predicted by the simulation. Figures 4.35-4.38 compare the measured lift coefficients to their simulations for the 4 additional spanwise locations. Figures 4.24 and 4.25 are the equivalent plots for the baseline case, $k_{c}=0.098$.

\subsubsection{Effect of Amplitude}

The tests were performed at different amplitudes to verify the linearity of the dynamics. Because the simulation uses linear assumptions, the best match between theory and experiments is expected at the lowest amplitudes. Results for $1^{\circ}$ and $4^{\circ}$ flap moment and deflection are shown in Figures 4.39 and 4.40. Results for lift coefficient are shown in Figures 4.41-4.44.

In general, while the $1^{\circ}$ amplitude runs should provide the best comparison with the linear theory, these runs have a larger relative error due to the forces and displacements being half as large, though the absolute error is similar for all cases. The $4^{\circ}$ runs tend to have higher error, both relative and absolute, compared to those of the smaller amplitudes. Example spanwise pressure data for $1^{\circ}$ and $4^{\circ}$ oscillations are shown in Figures 4.43 and 4.44 respectively, and represent the general effect of amplitude. The larger error in the $4^{\circ}$ runs suggests non-linear aerodynamics are playing a significant role in these tests. This non-linearity may be due to the low Reynolds number of this experiment or simply exceeding the range of the linear approximations in the underlying aerodynamic theory. 

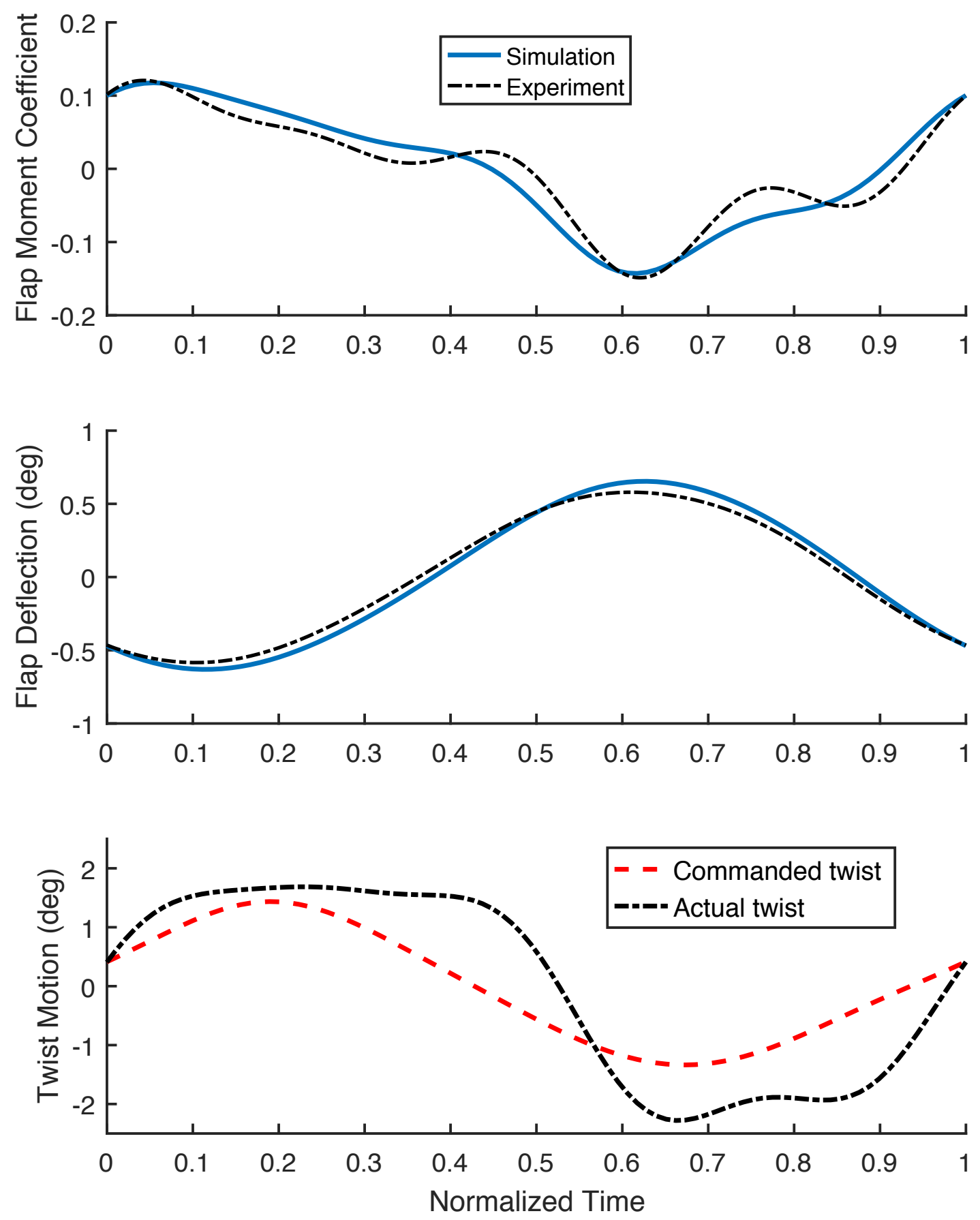

Figure 4.27: Simulated flap moment and motion for $k_{c}=0.13, V=10 \mathrm{~m} / \mathrm{s}$, and $A_{\phi_{t}}=2^{\circ}$. 

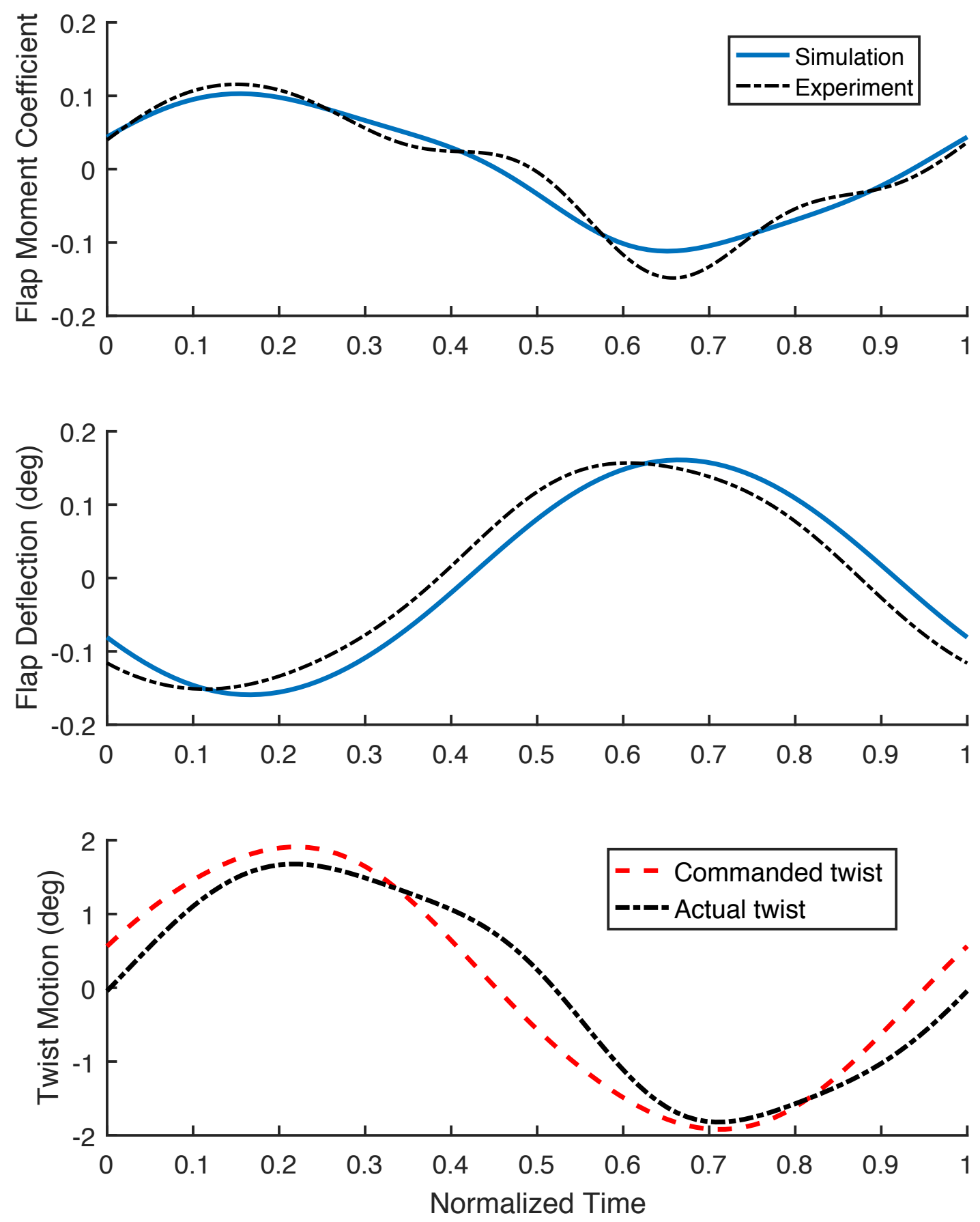

Figure 4.28: Simulated flap moment and motion for $k_{c}=0.22, V=10 \mathrm{~m} / \mathrm{s}$, and $A_{\phi_{t}}=2^{\circ}$. 

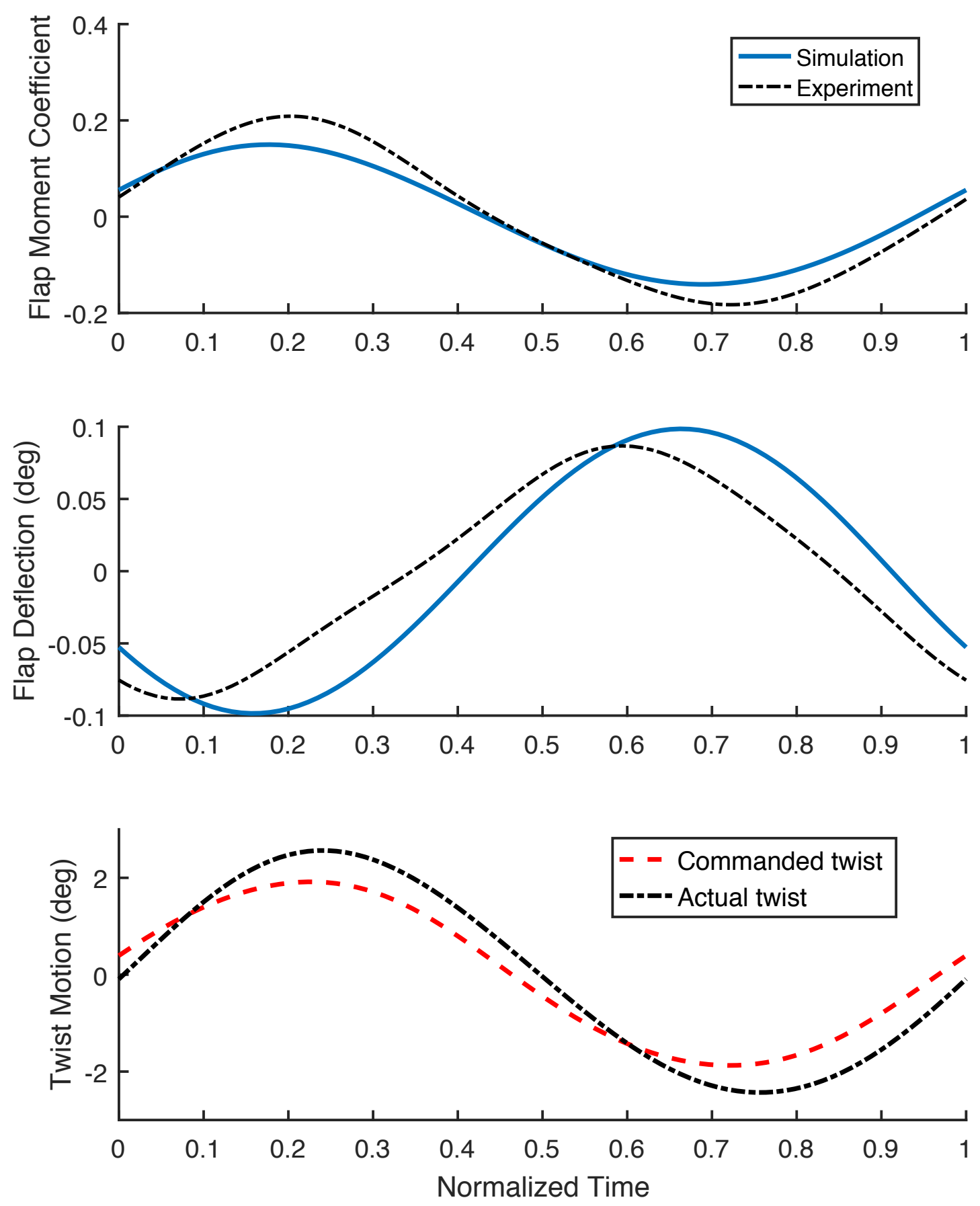

Figure 4.29: Simulated flap moment and motion for $k_{c}=0.31, V=10 \mathrm{~m} / \mathrm{s}$, and $A_{\phi_{t}}=2^{\circ}$. 

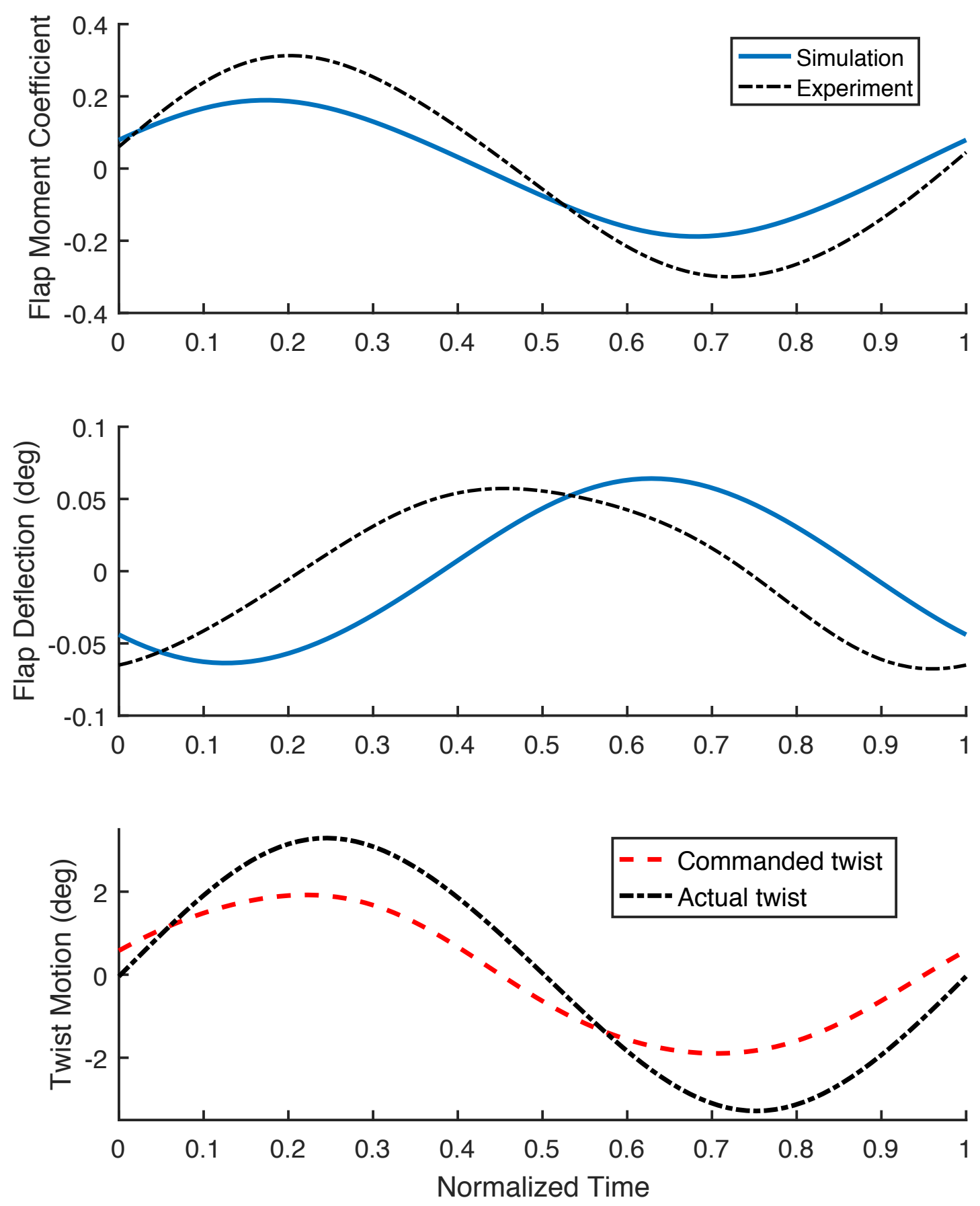

Figure 4.30: Simulated flap moment and motion for $k_{c}=0.39, V=10 \mathrm{~m} / \mathrm{s}$, and $A_{\phi_{t}}=2^{\circ}$. 


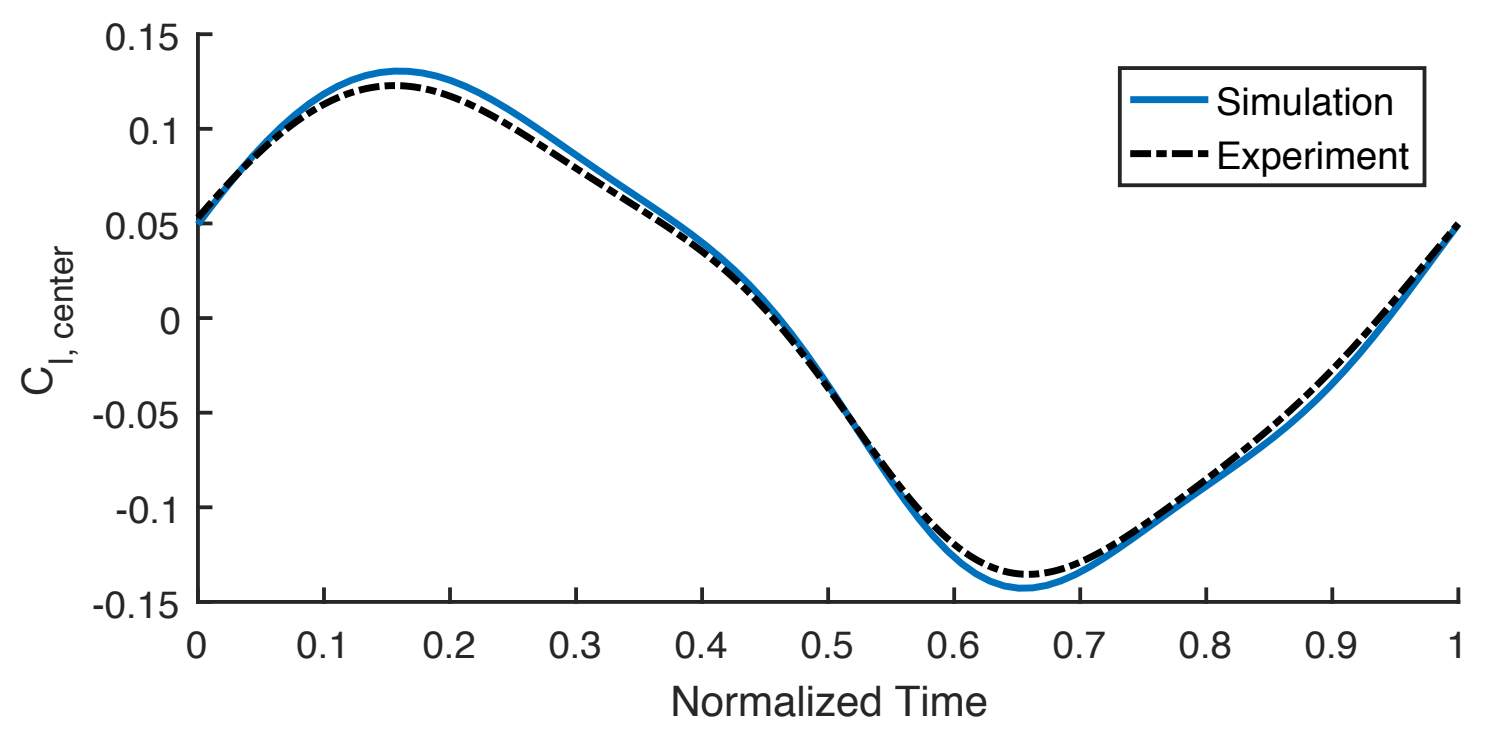

Figure 4.31: Center-span lift coefficient for $k_{c}=0.22, V=10 \mathrm{~m} / \mathrm{s}$, and $A_{\phi_{t}}=2^{\circ}$.

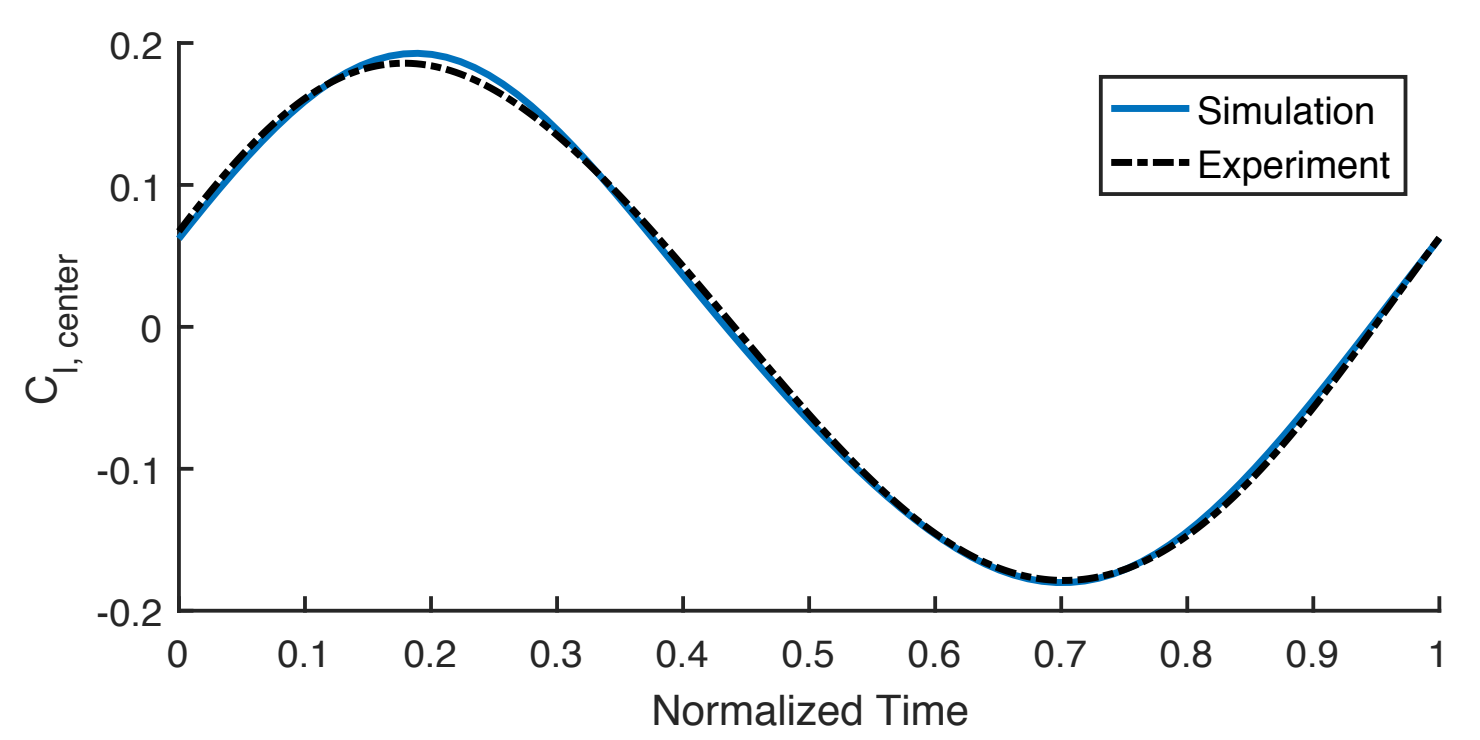

Figure 4.32: Center-span lift coefficient for $k_{c}=0.31, V=10 \mathrm{~m} / \mathrm{s}$, and $A_{\phi_{t}}=2^{\circ}$. 


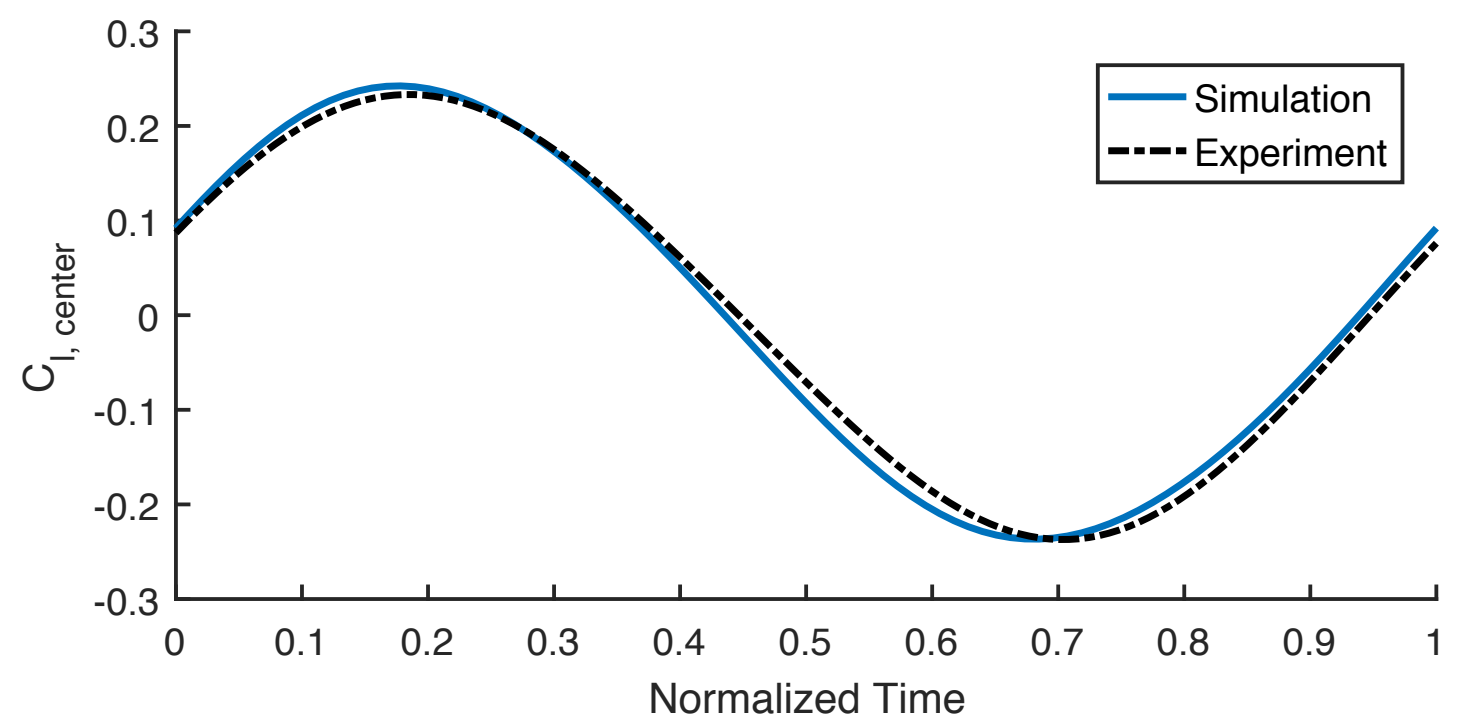

Figure 4.33: Center-span lift coefficient for $k_{c}=0.39, V=10 \mathrm{~m} / \mathrm{s}$, and $A_{\phi_{t}}=2^{\circ}$.

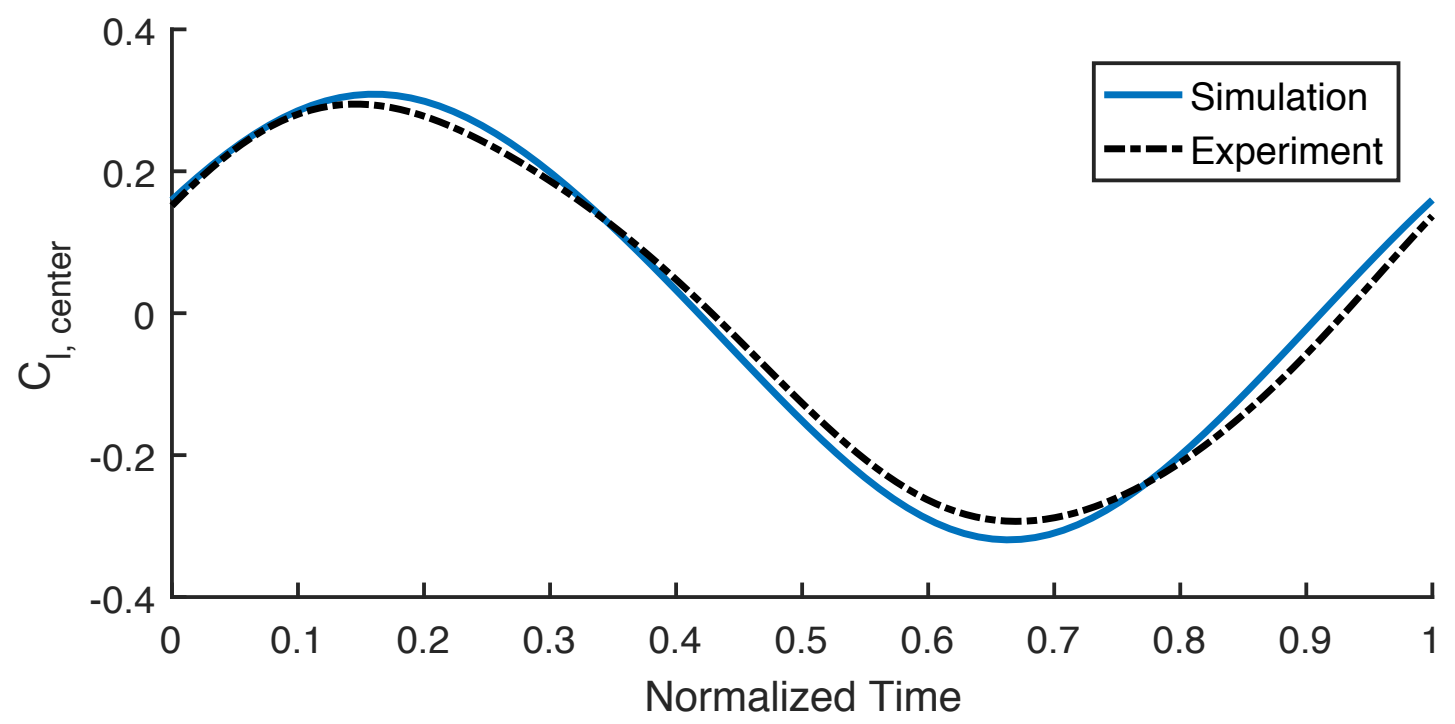

Figure 4.34: Center-span lift coefficient for $k_{c}=0.56, V=7 \mathrm{~m} / \mathrm{s}$, and $A_{\phi_{t}}=2^{\circ}$. The lower velocity is used to achieve a higher $k$ value at the maximum frequency of the motor. 

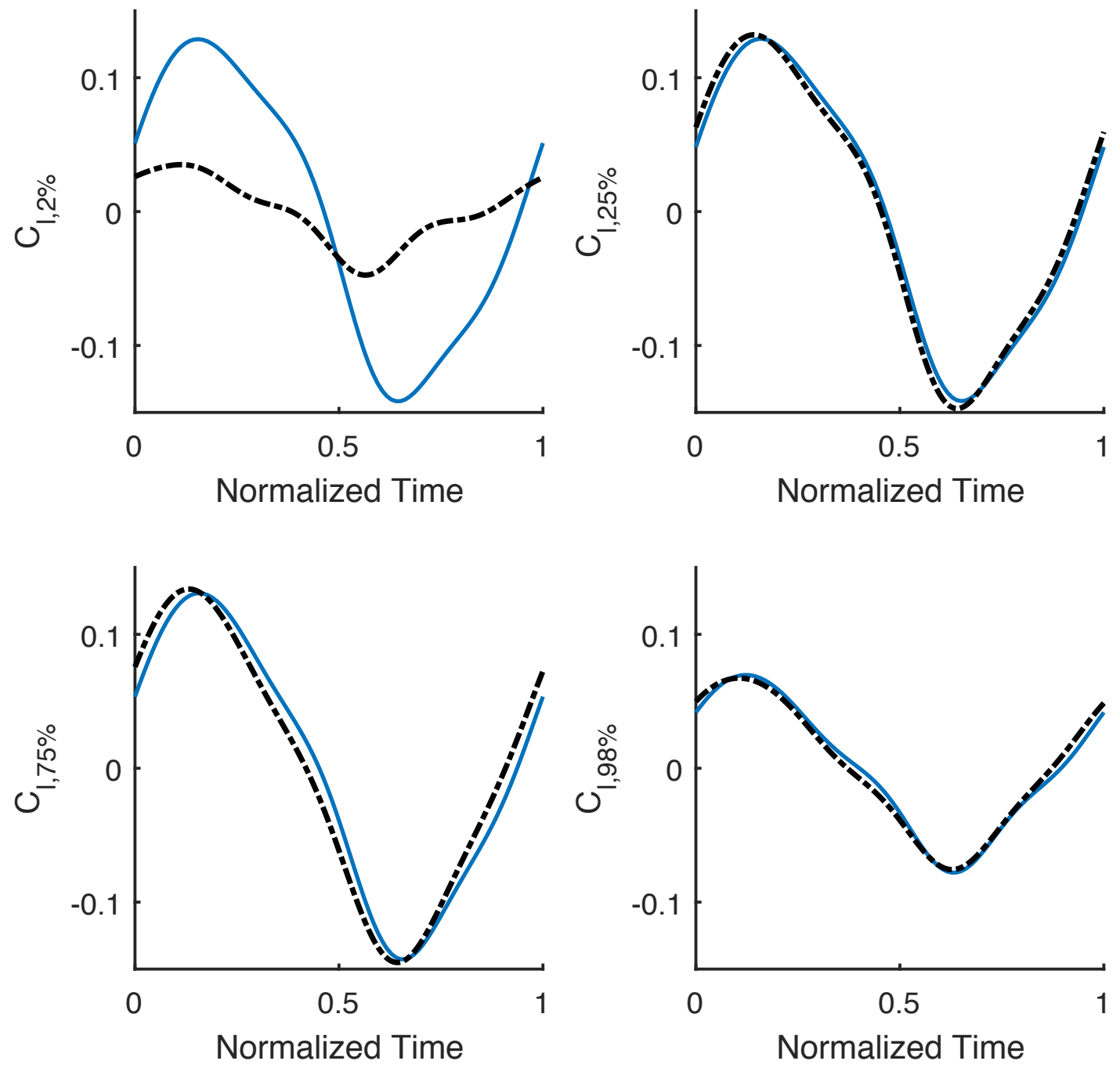

Figure 4.35: Simulated lift coefficients at various span locations for $k_{c}=0.22, V=$ $10 \mathrm{~m} / \mathrm{s}$, and $A_{\phi_{t}}=2^{\circ}$. 

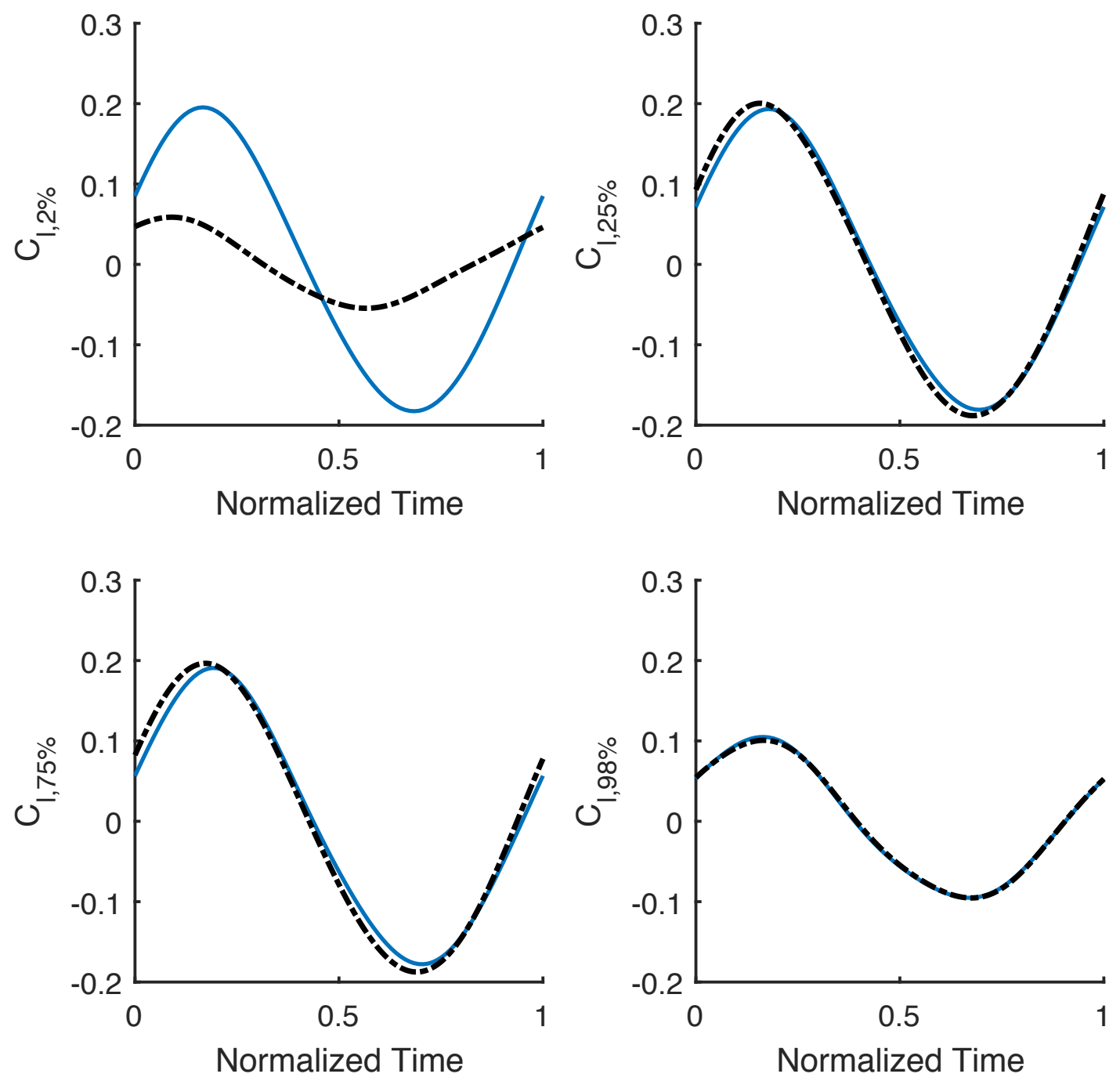

Figure 4.36: Simulated lift coefficients at various span locations for $k_{c}=0.31, V=$ $10 \mathrm{~m} / \mathrm{s}$, and $A_{\phi_{t}}=2^{\circ}$. 

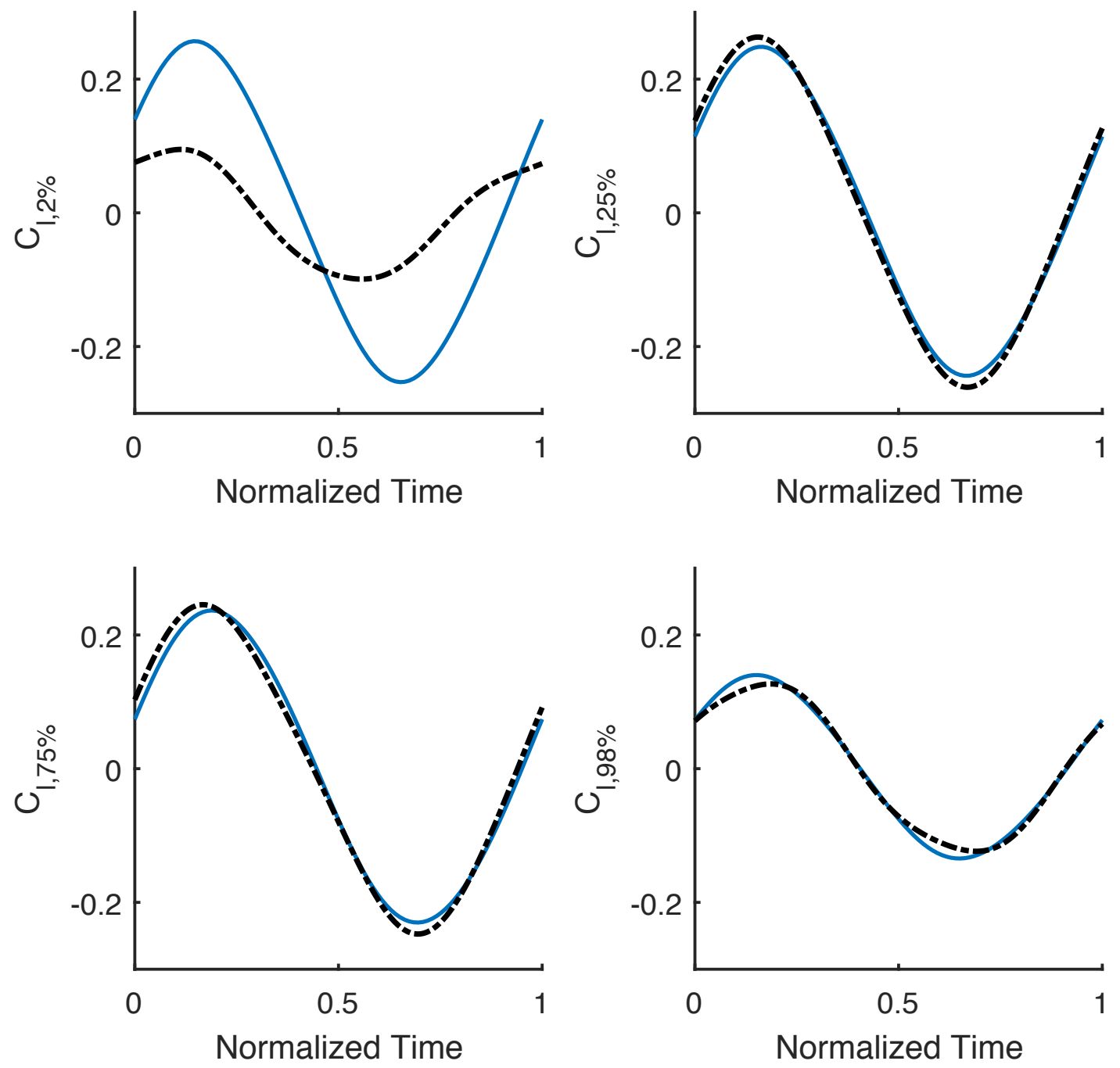

Figure 4.37: Simulated lift coefficients at various span locations for $k_{c}=0.39, V=$ $10 \mathrm{~m} / \mathrm{s}$, and $A_{\phi_{t}}=2^{\circ}$. 

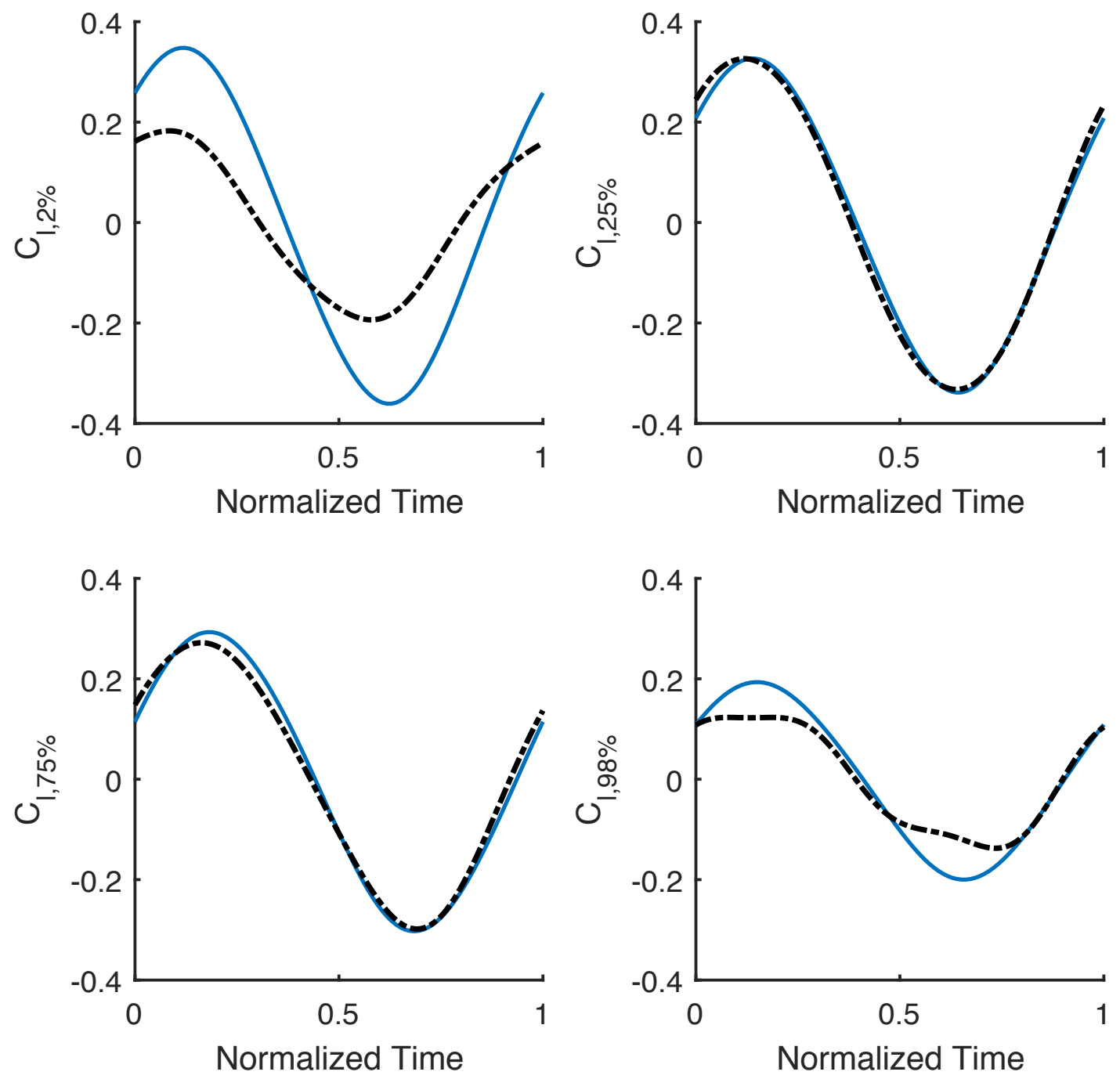

Figure 4.38: Simulated lift coefficients at various span locations for $k_{c}=0.56, V=7 \mathrm{~m} / \mathrm{s}$, and $A_{\phi_{t}}=2^{\circ}$. 

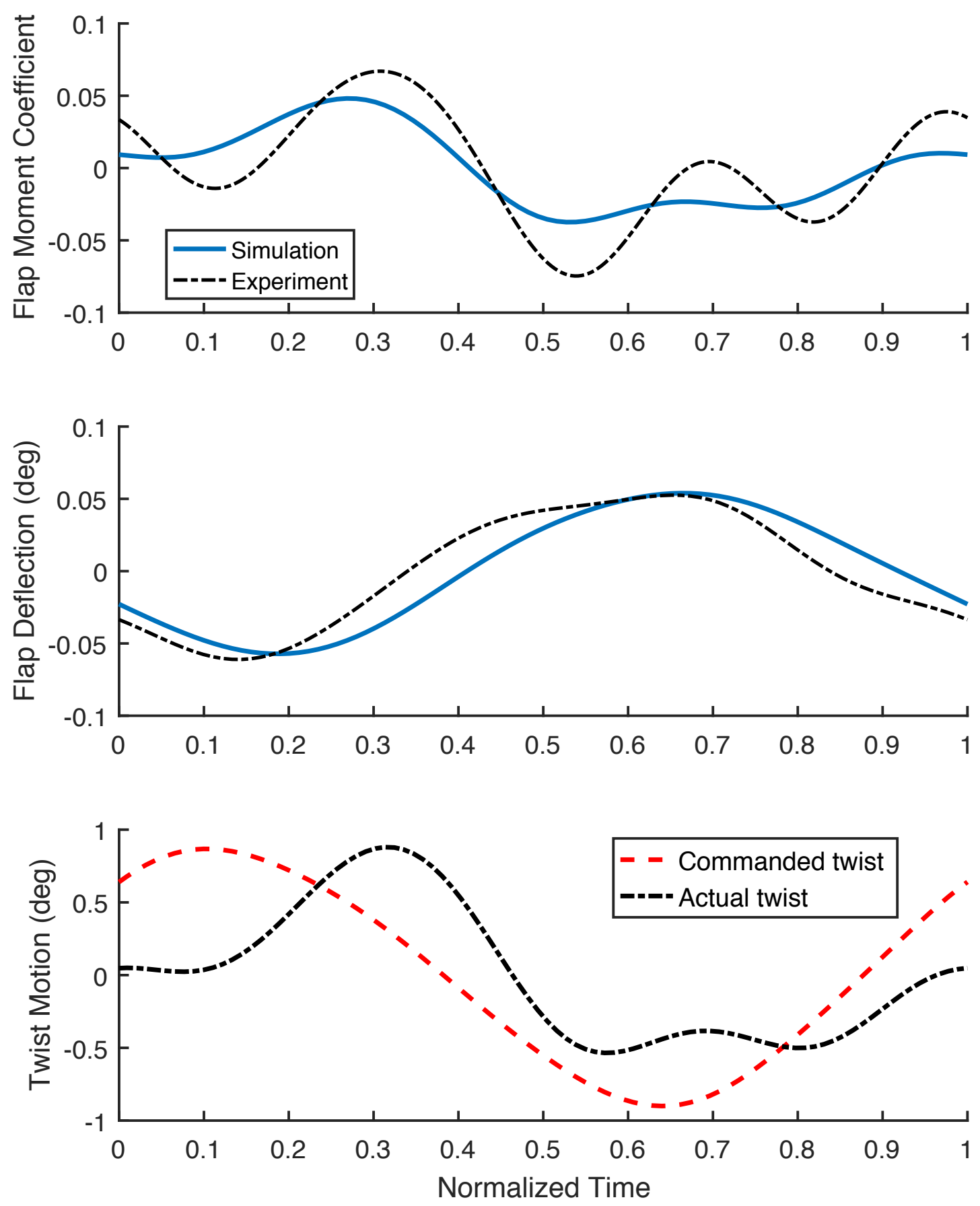

Figure 4.39: Simulated flap moment and motion for $k_{c}=0.22, V=10 \mathrm{~m} / \mathrm{s}$, and $A_{\phi_{t}}=1^{\circ}$. 

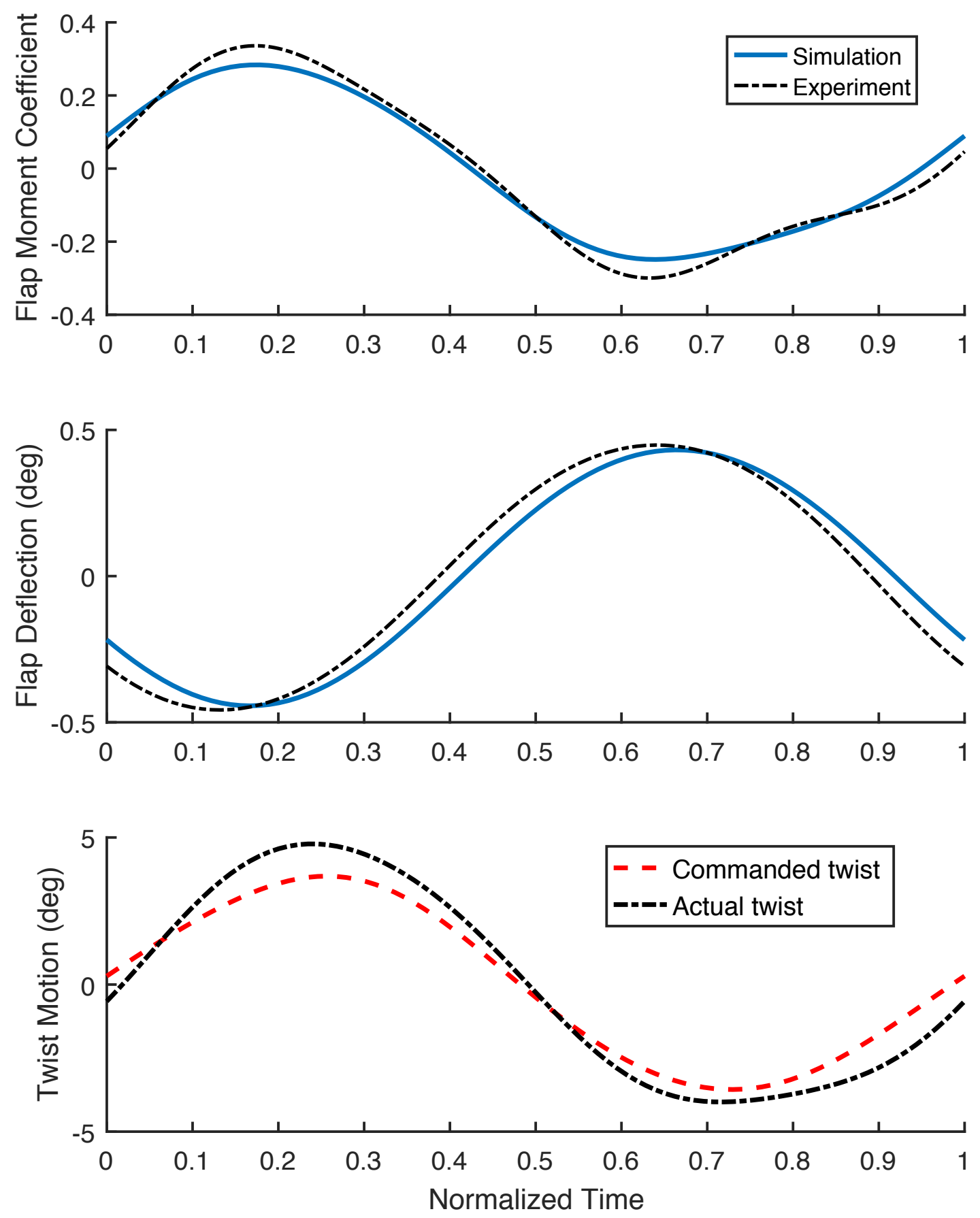

Figure 4.40: Simulated flap moment and motion for $k_{c}=0.22, V=10 \mathrm{~m} / \mathrm{s}$, and $A_{\phi_{t}}=4^{\circ}$ 


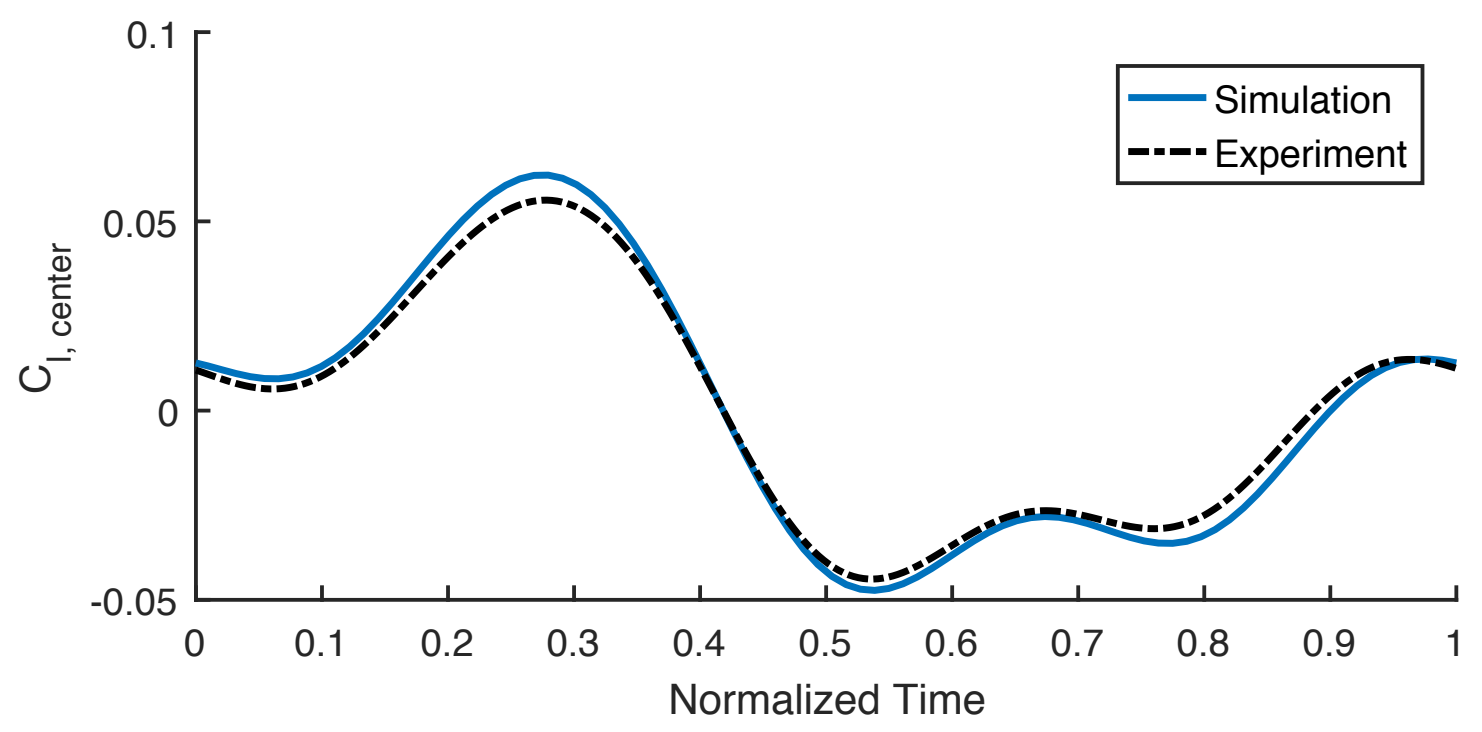

Figure 4.41: Simulated lift coefficients at the center-span $k_{c}=0.22, V=10 \mathrm{~m} / \mathrm{s}$, and $A_{\phi_{t}}=1^{\circ}$.

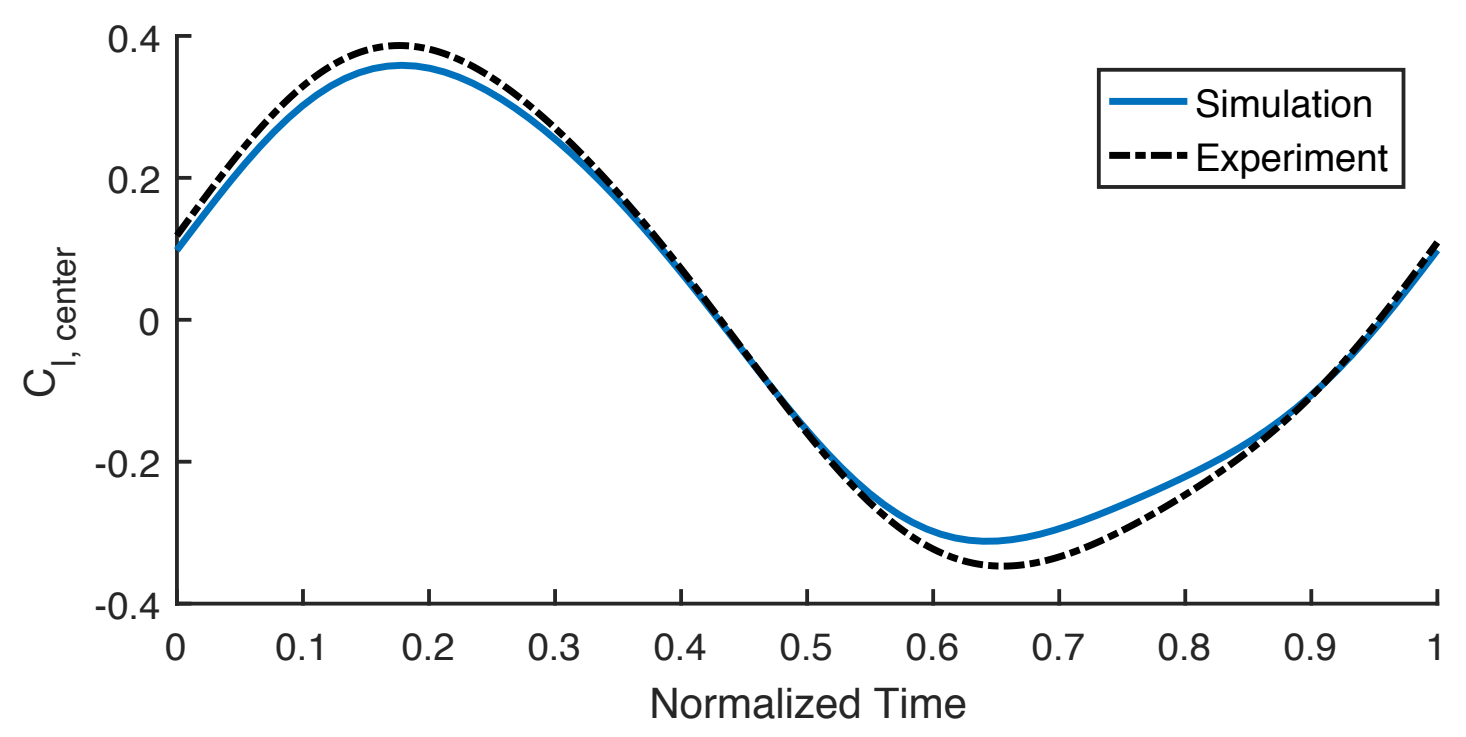

Figure 4.42: Simulated lift coefficients at the center-span $k_{c}=0.22, V=10 \mathrm{~m} / \mathrm{s}$, and $A_{\phi_{t}}=4^{\circ}$. 

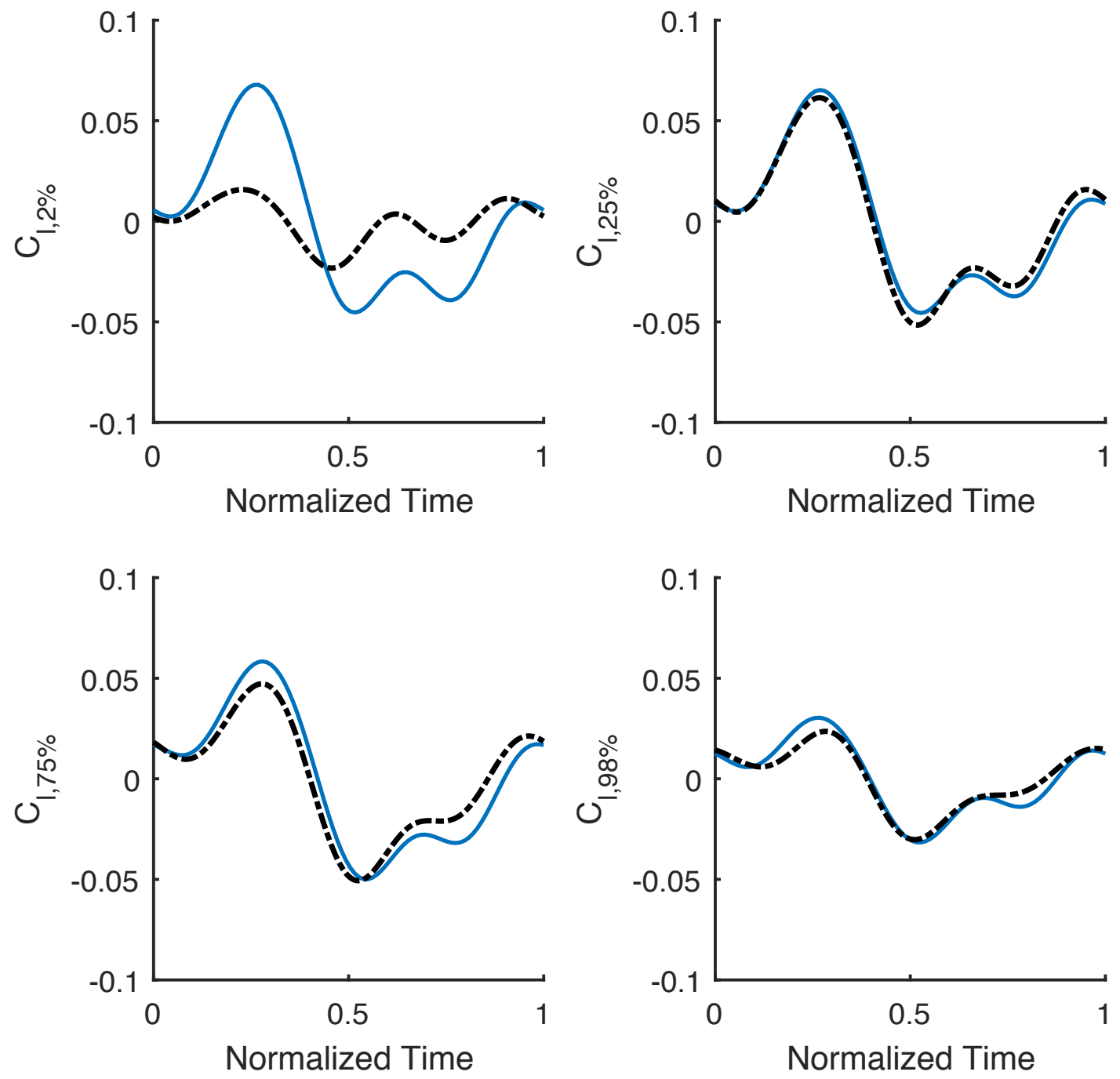

Figure 4.43: Simulated lift coefficients at various span locations for $k_{c}=0.22, V=$ $10 \mathrm{~m} / \mathrm{s}$, and $A_{\phi_{t}}=1^{\circ}$. 

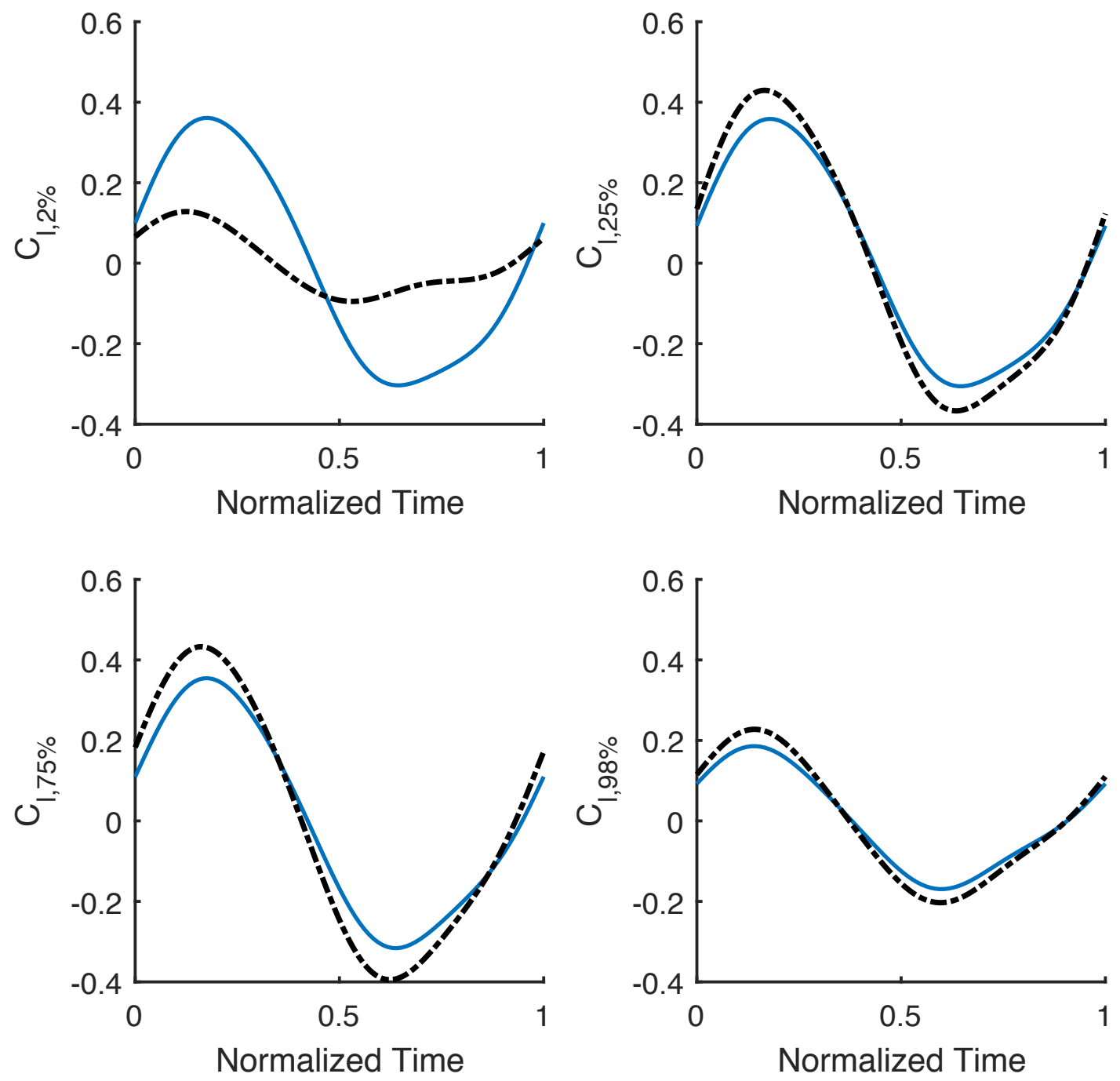

Figure 4.44: Simulated lift coefficients at various span locations for $k_{c}=0.22, V=$ $10 \mathrm{~m} / \mathrm{s}$, and $A_{\phi_{t}}=4^{\circ}$. 


\subsection{Comparison to Other Aerodynamic Models}

Results from the $k_{c}=0.098, k_{c}=0.22$, and $k_{c}=0.39$ cases are compared to two alternate aerodynamic models: strip theory using Theodorsen's aerodynamics and quasisteady lifting-line theory. Strip theory models the blade as a finite number of 2D airfoil "strips" whose lift is independent from one another (i.e., blade sections do not induce downwash on other sections). The model developed here, based on Wagner aerodynamics, is included as a comparison.

Figures 4.45-4.47 compare the predicted deflection and integrated moment for each of these models with the experiment. Theodorsen strip theory and the Wagner-based model give similar results, though strip theory tends to slightly overestimate the flap deflection at $k_{c}$ values near 0.22 , and would therefore overestimate fatigue. Part of the reason appears to be that strip theory significantly overestimates the sectional lift near the outboard end of the blade, as seen in Figures 4.51-4.53. This effect becomes more pronounced at low $k_{c}$, as the sectional lift becomes dominated by the circulatory component of lift. Despite this, strip theory does not significantly overestimate the flap deflection at the lowest $k_{c}$ value of 0.098. The cause of this is unknown.

Figures 4.48-4.50 compare the predicted sectional lift for each of these models with the experiment. As expected, the quasi-steady model performs progressively worse as $k$ increases. Theodorsen strip theory gives nearly identical results to the Wagner-based lifting line model for sectional lift at the center span. This is because aerodynamics at the center-span can be assumed to be mostly 2D. 

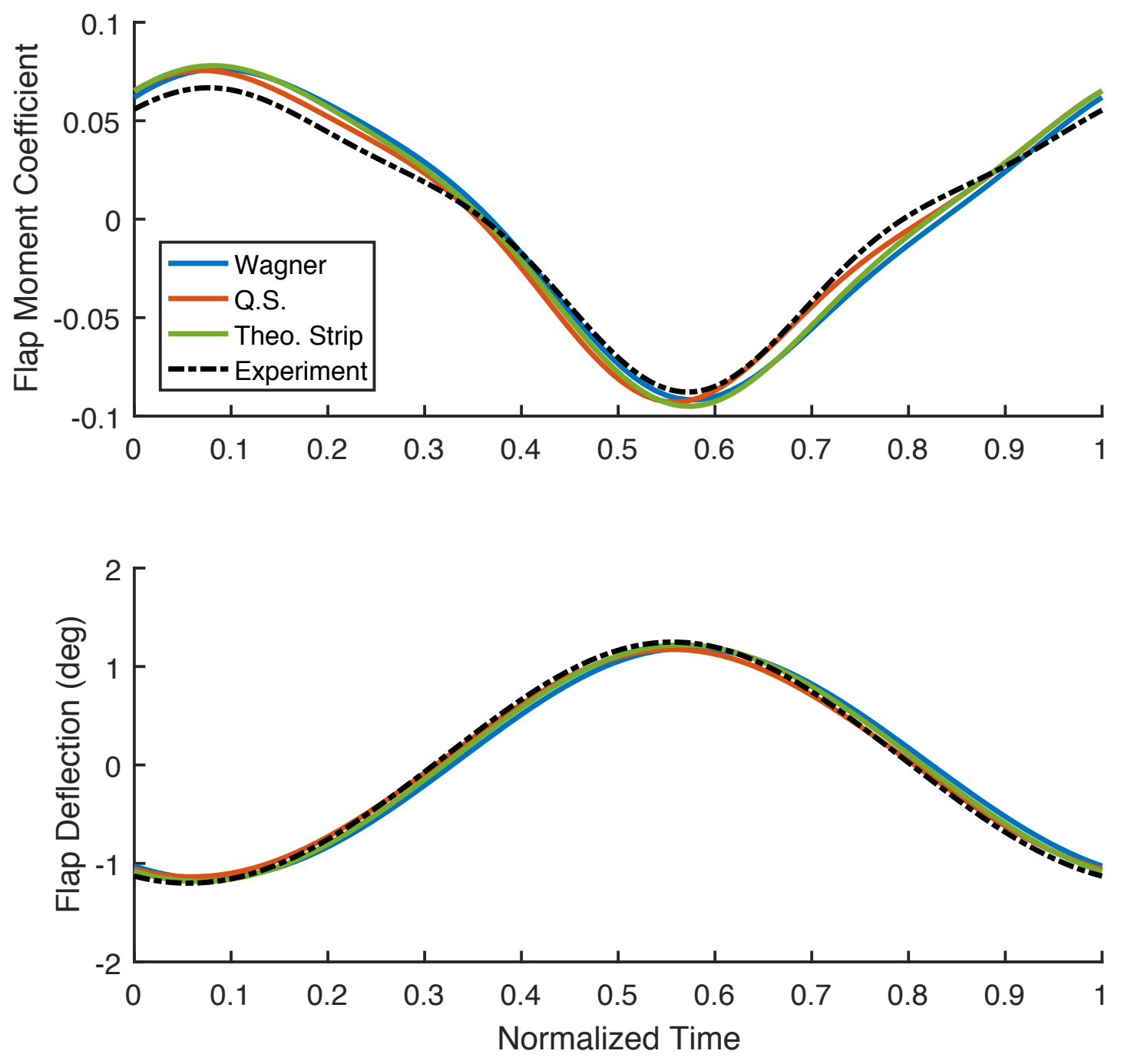

Figure 4.45: Moment and motion for $k_{c}=0.098, V=10 \mathrm{~m} / \mathrm{s}$, and $A_{\phi_{t}}=2^{\circ}$ using 3 different aerodynamic models. 

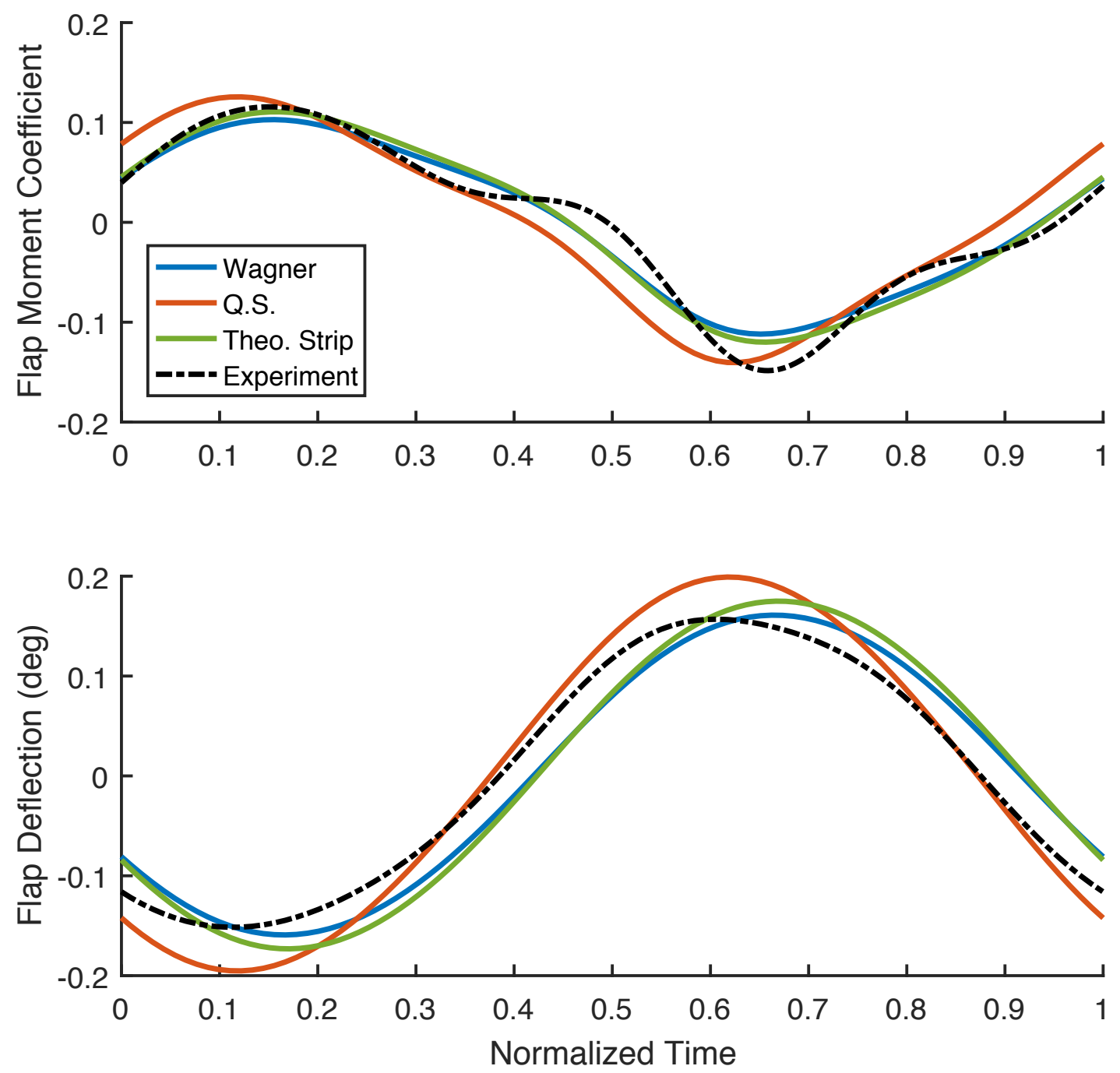

Figure 4.46: Moment and motion for $k_{c}=0.22, V=10 \mathrm{~m} / \mathrm{s}$, and $A_{\phi_{t}}=2^{\circ}$ using 3 different aerodynamic models. 

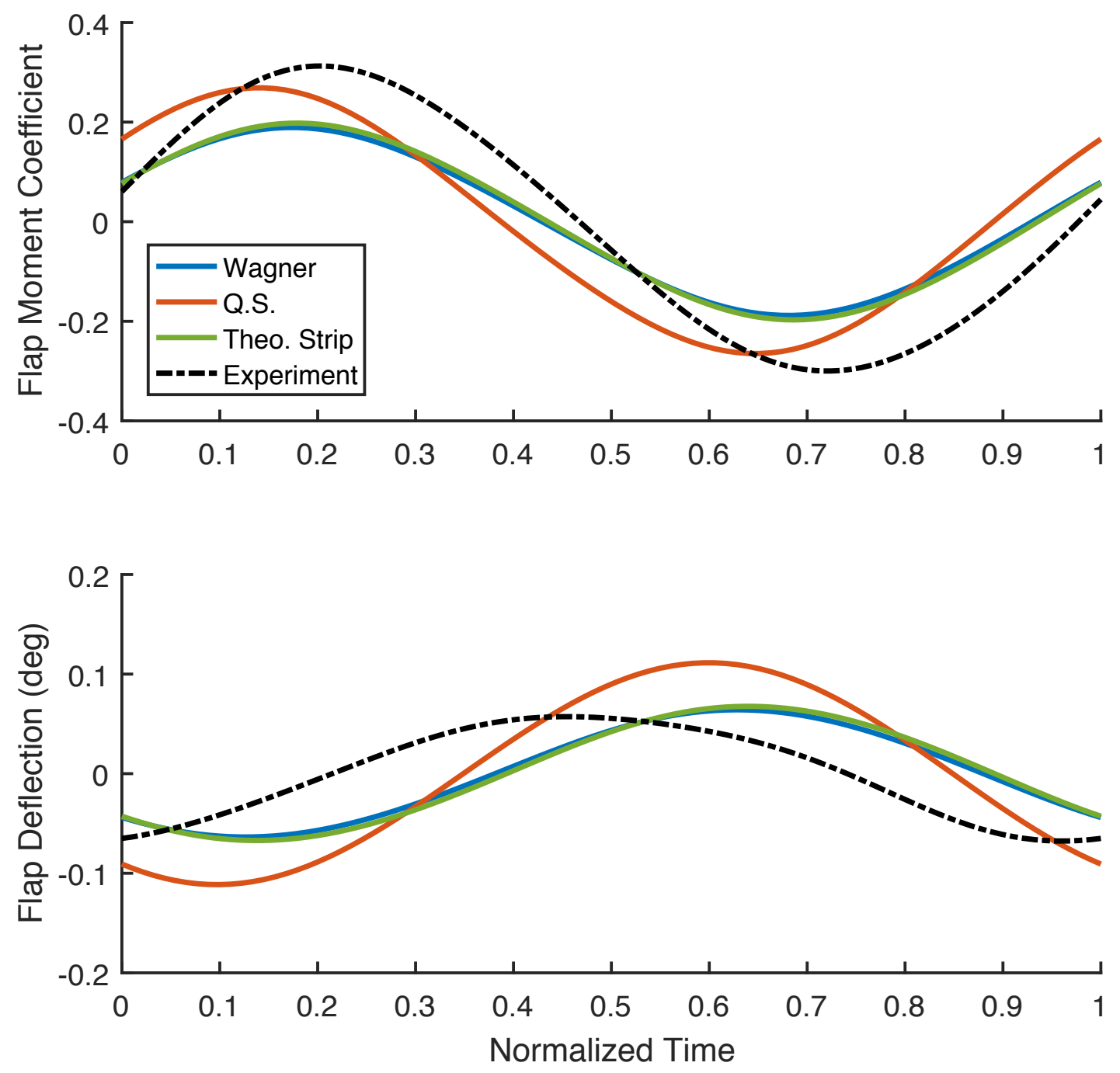

Figure 4.47: Moment and motion for $k_{c}=0.39, V=10 \mathrm{~m} / \mathrm{s}$, and $A_{\phi_{t}}=2^{\circ}$ using 3 different aerodynamic models. 


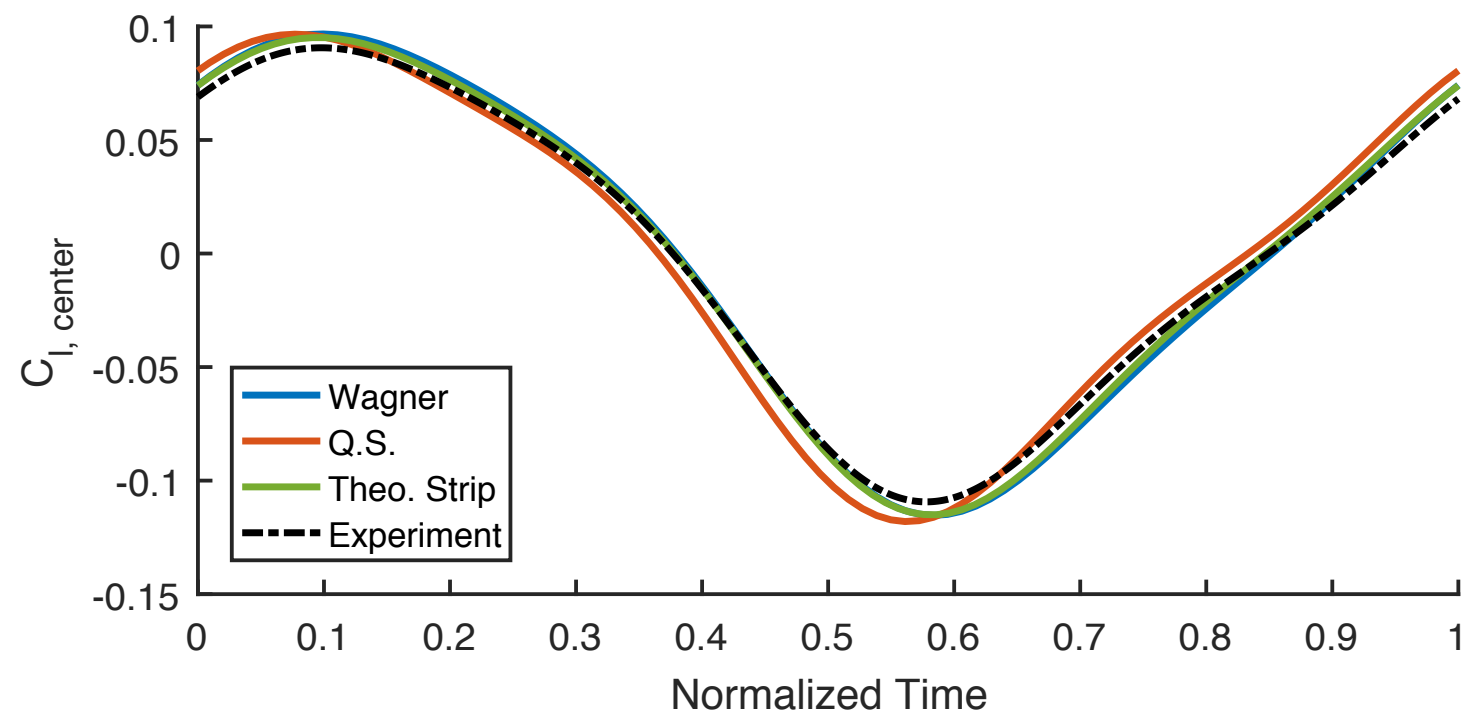

Figure 4.48: Center-span sectional lift for $k=0.098, V=10 \mathrm{~m} / \mathrm{s}$, and $A_{\phi_{t}}=2^{\circ}$ using 3 different aerodynamic models.

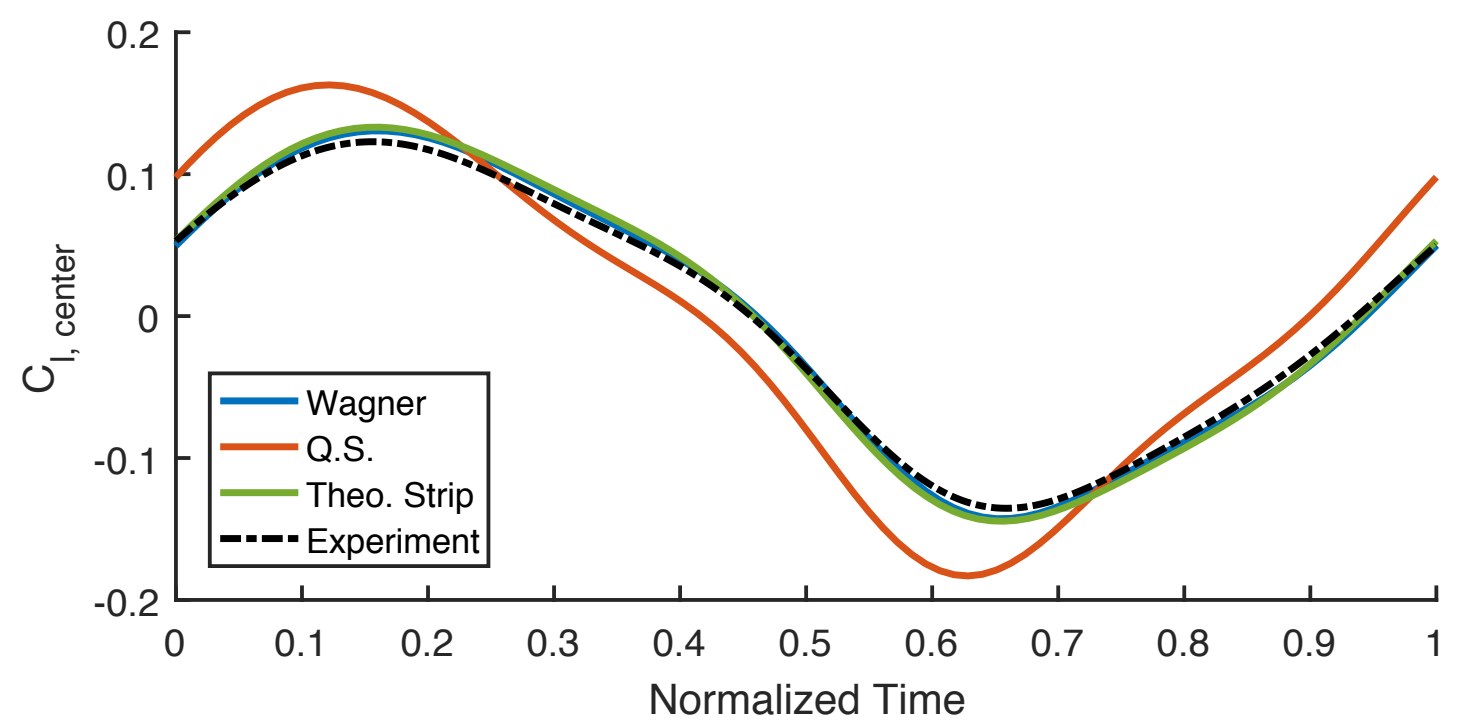

Figure 4.49: Center-span sectional lift for $k=0.22, V=10 \mathrm{~m} / \mathrm{s}$, and $A_{\phi_{t}}=2^{\circ}$ using 3 different aerodynamic models. 


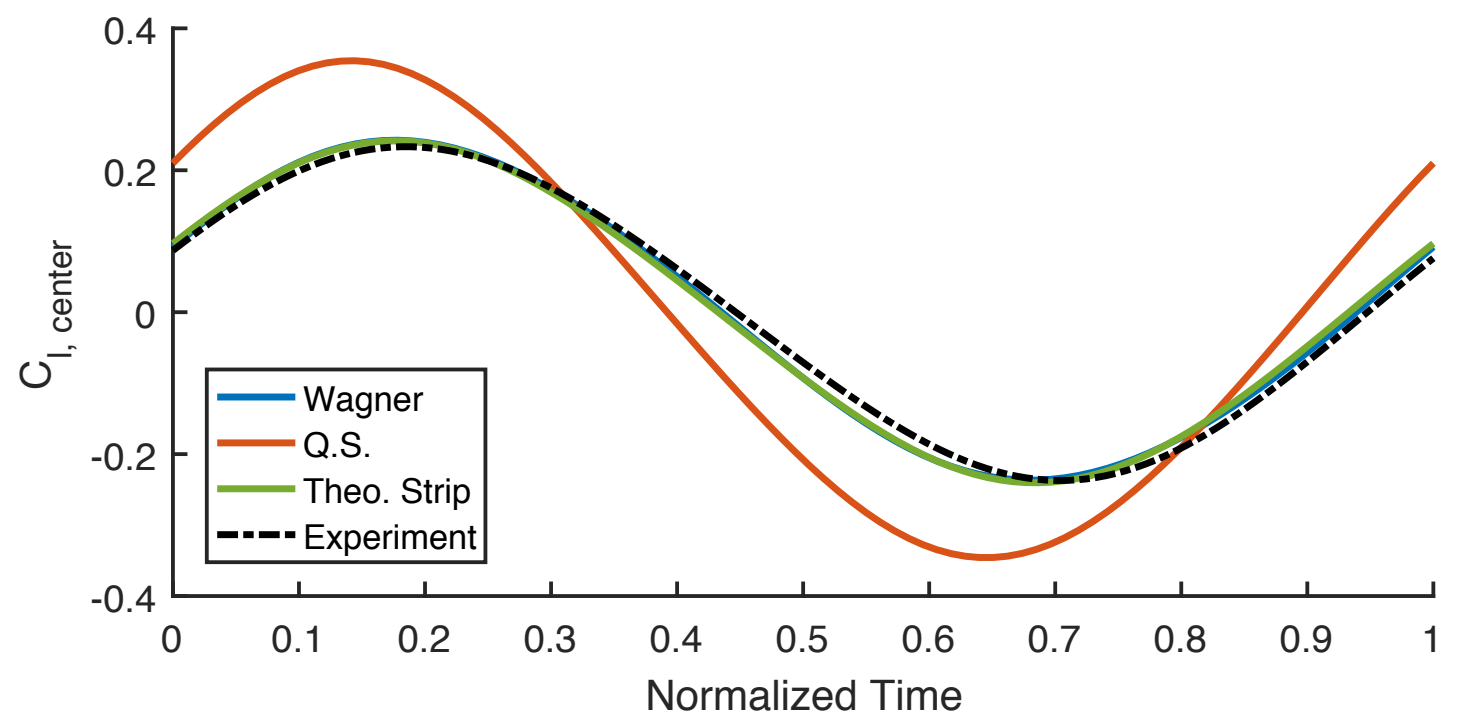

Figure 4.50: Center-span sectional lift for $k=0.39, V=10 \mathrm{~m} / \mathrm{s}$, and $A_{\phi_{t}}=2^{\circ}$ using 3 different aerodynamic models. 

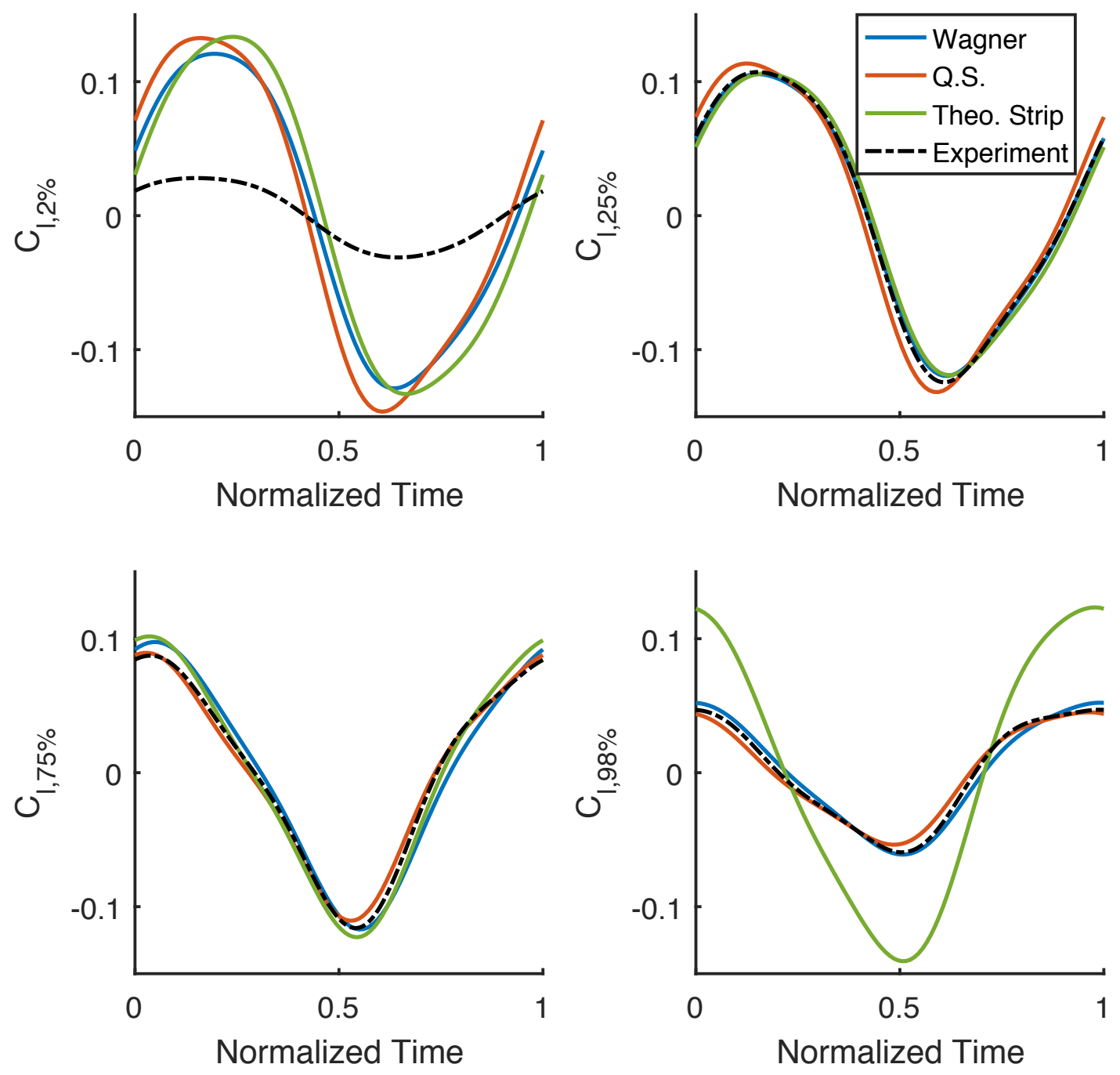

Figure 4.51: Sectional lift at various spanwise locations for $k_{c}=0.098, V=10 \mathrm{~m} / \mathrm{s}$, and $A_{\phi_{t}}=2^{\circ}$ using 3 different aerodynamic models. 

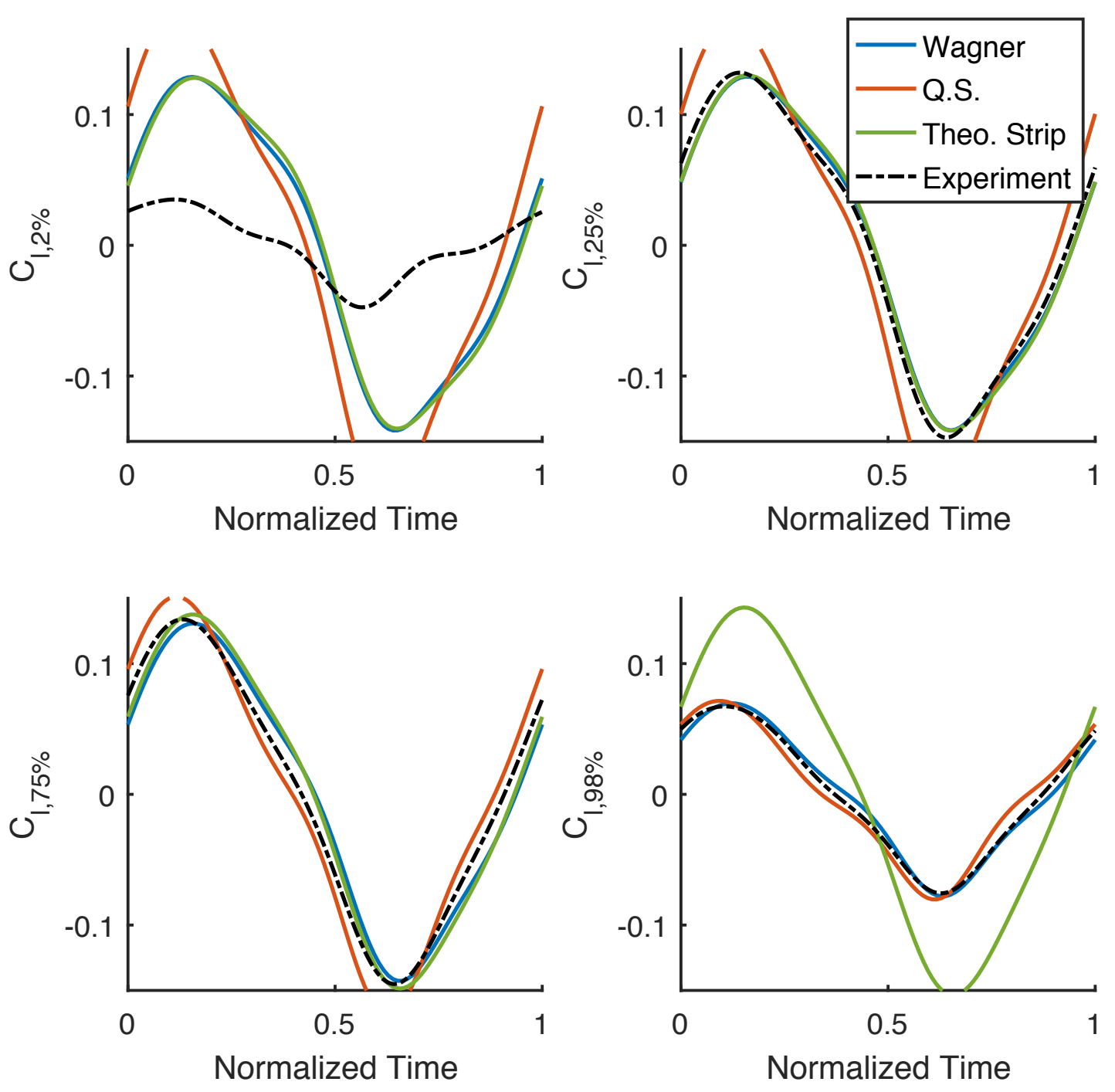

Figure 4.52: Sectional lift at various spanwise locations for $k_{c}=0.22, V=10 \mathrm{~m} / \mathrm{s}$, and $A_{\phi_{t}}=2^{\circ}$ using 3 different aerodynamic models. 

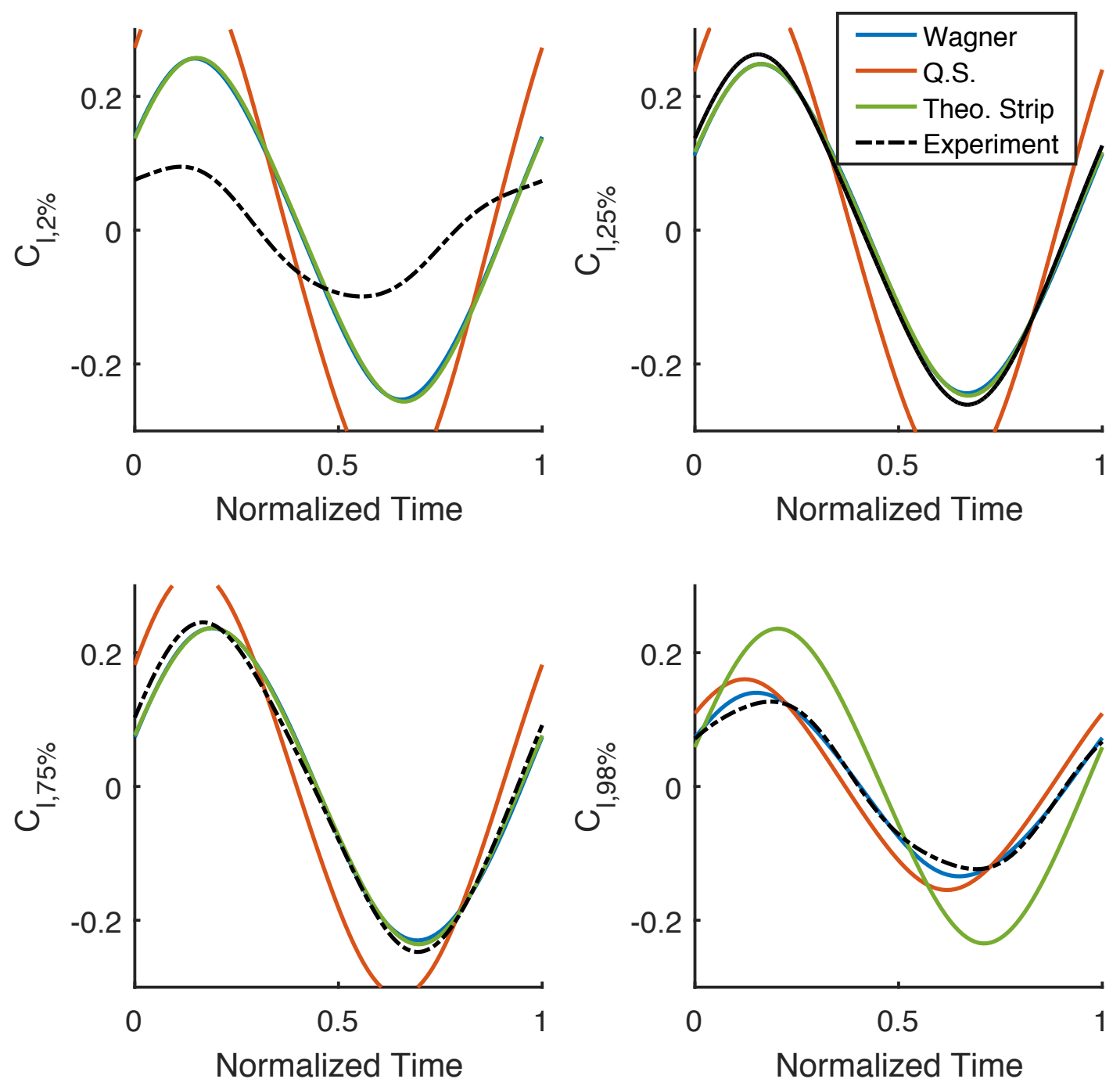

Figure 4.53: Sectional lift at various spanwise locations for $k_{c}=0.39, V=10 \mathrm{~m} / \mathrm{s}$, and $A_{\phi_{t}}=2^{\circ}$ using 3 different aerodynamic models. 


\section{CONCLUSIONS AND FUTURE WORK}

This work describes the development of a dynamic aeroelastically-scaled turbine blade, capable of flapping and twisting motion, for wind tunnel tests. This model was subjected to various amplitudes and frequencies of disturbances to determine the resulting flap motion and determine which conditions would contribute most to fatigue. A predictive model based on 3D unsteady aerodynamics was developed for simulating the response of the test article to aerodynamic disturbances. The predictive model outputs were compared to the experiment and found to be in good agreement between simulation and experiment. The 3D aerodynamic model demonstrates improvement in estimating the amplitude of the resulting motion over traditional strip theory.

A finite-span, rigid blade with an elastic base, compliant in flap and twist, was constructed with elasticity and mass parameters characteristic of a full-scale blade. Unsteady aerodynamic disturbances can be applied using an actuator motor on the twist spring. This allows the model to respond elastically to aerodynamic disturbances, instead of being directly driven to a prescribed motion using motors alone. The model can be easily adjusted to simulate different types on large-scale wind turbine blades by changing the stiffness of the spring and/or shifting the elastic axis. This blade will also serve as a test platform for future aeroelastic tests and possible active control experiments.

A time-domain, linear simulation model for the test article was developed and validated with wind-tunnel experiments. This model can be readily adapted to simulate many different types of blades, including continuously flexible blades on full-scale turbines. This linear, time-domain model will be useful for future active controls research.

The simulated loads and motions were compared to those measured in the wind tunnel experiments. These comparisons show good agreement between model and measurement, 
though there is some disagreement for the predicted flap deflection at high frequencies. However, the deflections are so small at these frequencies that it is unlikely the experiment is capable of reliably measuring them, and such small deflections would not be of interest to the study of fatigue. Comparisons to other aerodynamics models show that Wagnerbased lifting line model performs better than or equal to the widely used Theodorsenbased strip theory. Strip theory tends to overestimate the fatigue-inducing motion at certain frequencies, in part due to its overestimate of lift near the outboard end of the blade.

Though the predictive model is capable of estimating twist deformation and the coupling between flap and twist motion, a design flaw in the elastic base of the test article precludes comparison between these two degrees of freedom. The unsteady loads from edgewise loads were found to have a significant effect on the elastic deformation of the twist degree of freedom. The elastic base will need to be redesigned for future tests.

The rigid blade and the 3D aeroelastic model will serve as a platform for continuing research into turbine fatigue motion and active load alleviation. The following goals of future work have been identified:

1. The elastic base must be redesigned. The base presented in this work was chosen for its ease of construction, low moment of inertia, and relative simplicity, but it ultimately failed to properly simulate the elastic twist response. This work would have greatly benefited from a proper gimbal with interchangeable torsional springs. Future tests should use a 3-axis gimbal, for edge, flap, and twist motion, with a custom-manufactured torsional spring on each axis. This will be significantly harder to manufacture while keeping the inertia negligible, but it will allow much easier customization for simulation of different types of turbine blades. Depending on the design of this base, a non-linear structural simulation model may be needed.

2. The accelerometer used in this test is non lab-grade equipment. A validation study 
should be performed to confirm the frequency response and calibration for this sensor.

3. The vortex at the base of the blade should be mitigated. Extending the blade surface down into the hole in the tunnel floor may solve this issue.

4. Edgewise motion was ignored in this work. This choice was made to simplify the elastic base for the test article, and was considered acceptable due to the small forcing in this direction. Though the forcing in this direction is much smaller than that of flapwise motion, the damping of this mode is small and significantly contributes to fatigue. Now that the simulation model has been validated for flapwise response, future work should consider incorporating the edgewise degree of freedom as well.

5. Aerodynamic disturbances were applied by rotating the elastic base. This is similar to how a blade would respond to gusts, but it is not identical. Future wind tunnel experiments should implement a gust generator for validation of Küssner aerodynamics or other gust models.

6. The rigid blade could be refitted with control surfaces-either micro-tabs on the suction side, or a trailing edge flap. These could be used in conjunction with gust sensors such as those used by Babbar [8] to demonstrate an active load alleviation controller.

7. The spring for the twist degree of freedom could be replaced with an actuator motor to create a cyber-physical system. The motor would apply torque proportional to measured angular displacement and would act analogously to a spring. This would not only ease customization of twist stiffness, but could also be used to simulate various degrees of bend-twist coupling, which could be used for passive load alleviation. 


\section{REFERENCES}

[1] S. Lindenberg, 20\% Wind Energy By 2030: Increasing Wind Energy's Contribution to US Electricity Supply. Diane Publishing, 2009.

[2] J. G. Njiri and D. Söffker, "State-of-the-art in wind turbine control: Trends and challenges," Renewable and Sustainable Energy Reviews, vol. 60, pp. 377 - 393, 2016.

[3] M. O. L. Hansen, J. N. Sørensen, S. Voutsinas, N. Sørensen, and H. A. Madsen, "State of the art in wind turbine aerodynamics and aeroelasticity," Progress in aerospace sciences, vol. 42, no. 4, pp. 285-330, 2006.

[4] D. A. Simms, S. Schreck, M. Hand, and L. Fingersh, NREL unsteady aerodynamics experiment in the NASA-Ames wind tunnel: a comparison of predictions to measurements. National Renewable Energy Laboratory Golden, CO, USA, 2001.

[5] D. Simms, M. Hand, L. Fingersh, and D. Jager, "Unsteady aerodynamics experiment phases ii-iv test configurations and available data campaigns," National Renewable Energy Laboratory, Colorado, vol. 3, 1999.

[6] L. Y. Pao and K. E. Johnson, "A tutorial on the dynamics and control of wind turbines and wind farms," in American Control Conference, 2009. ACC'09., pp. 2076-2089, IEEE, 2009.

[7] P. Fleming, A. Scholbrock, A. Jehu, S. Davoust, E. Osler, A. Wright, and A. Clifton, "Field-test results using a nacelle-mounted lidar for improving wind turbine power capture by reducing yaw misalignment," in Journal of Physics: Conference Series, vol. 524, p. 012002, IOP Publishing, 2014.

[8] Y. Babbar, Aeroelastic Response of a Typical Wing Section under Nonlinearities and Gust Loads. PhD thesis, 2015. 
[9] J. M. Jonkman, S. Butterfield, W. Musial, and G. Scott, Definition of a 5-MW reference wind turbine for offshore system development. National Renewable Energy Laboratory Golden, CO, 2009.

[10] J. Dugundji and J. M. Calligeros, "Similarity laws for aerothermoelastic testing," Journal of the AeroSpace Sciences, vol. 29, no. 8, pp. 935-950, 1962.

[11] H. Bergh and H. Tijdeman, "Theoretical and experimental results for the dynamic response of pressure measuring systems," tech. rep., Nationaal Lucht-en Ruimtevaartlaboratorium, 1965.

[12] S. A. Whitmore, M. D. Wilson, and S. D. Eilers, "Novel technique for reconstructing high-frequency transient rocket chamber-pressure measurements," Journal of Spacecraft and Rockets, vol. 47, no. 3, p. 427, 2010.

[13] D. H. Hodges and G. A. Pierce, Introduction to structural dynamics and aeroelasticity, vol. 15. cambridge university press, 2011.

[14] T. V. Karman, "Airfoil theory for non-uniform motion," Journal of the Aeronautical Sciences, vol. 5, no. 10, pp. 379-390, 1938.

[15] J. G. Leishman, "Challenges in modelling the unsteady aerodynamics of wind turbines," Wind energy, vol. 5, no. 2-3, pp. 85-132, 2002.

[16] J. Boutet and G. Dimitriadis, "Unsteady lifting line theory using the wagner function," in Proceedings of the 55th AIAA Aerospace Sciences Meeting, pp. AIAA-2017, AIAA, 2017. 